Florida International University

FIU Digital Commons

FIU Electronic Theses and Dissertations

University Graduate School

5-20-2016

\title{
Combined Computational-Experimental Design of High-Temperature, High-Intensity Permanent Magnetic Alloys with Minimal Addition of Rare- Earth Elements
}

Rajesh Jha

Florida International University, rjha001@fiu.edu

DOI: $10.25148 /$ etd.FIDC000704

Follow this and additional works at: https:// digitalcommons.fiu.edu/etd

Part of the Categorical Data Analysis Commons, Design of Experiments and Sample Surveys Commons, Metallurgy Commons, Numerical Analysis and Scientific Computing Commons, Other Materials Science and Engineering Commons, Statistical Methodology Commons, and the Theory and Algorithms Commons

\section{Recommended Citation}

Jha, Rajesh, "Combined Computational-Experimental Design of High-Temperature, High-Intensity Permanent Magnetic Alloys with Minimal Addition of Rare-Earth Elements" (2016). FIU Electronic Theses and Dissertations. 2621.

https://digitalcommons.fiu.edu/etd/2621

This work is brought to you for free and open access by the University Graduate School at FIU Digital Commons. It has been accepted for inclusion in FIU Electronic Theses and Dissertations by an authorized administrator of FIU Digital Commons. For more information, please contact dcc@fiu.edu. 


\section{FLORIDA INTERNATIONAL UNIVERSITY}

Miami, Florida

\section{COMBINED COMPUTATIONAL-EXPERIMENTAL DESIGN OF HIGH TEMPERATURE, HIGH-INTENSITY PERMANENT MAGNETIC ALLOYS WITH MINIMAL ADDITION OF RARE-EARTH ELEMENTS}

A dissertation submitted in partial fulfillment of the requirements for the degree of DOCTOR OF PHILOSOPHY

in

MATERIALS SCIENCE AND ENGINEERING

by

Rajesh Jha 
To: Interim Dean Ranu Jung

College of Engineering and Computing

This dissertation, written by Rajesh Jha, and entitled Combined ComputationalExperimental Design of High Temperature, High-Intensity Permanent Magnetic Alloys With Minimal Addition of Rare-Earth Elements, having been approved in respect to style and intellectual content, is referred to you for judgement.

We have read this thesis and recommend that it be approved.

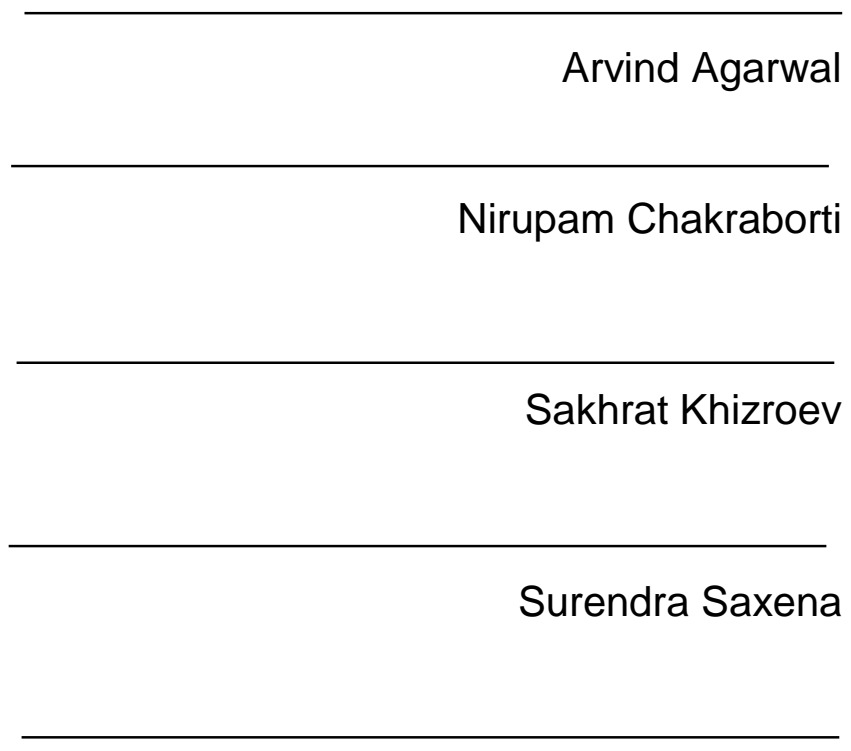

George S. Dulikravich, Major Professor

Date of Defense: May 20, 2016

The dissertation of Rajesh Jha is approved.

Interim Dean Ranu Jung College of Engineering and Computing

Andrés G. Gil

Vice President for Research and Economic Development and Dean of the University Graduate School

Florida International University, 2016 


\section{COPYRIGHT}

This work resulted in several journal articles and refereed conference proceedings. I have used part of this work in my thesis.

Following is the list of papers that have been published or accepted for publication: [Book Chapter]

1. Jha, R., Dulikravich, G. S., Colaço, M.J., Fan, M., Shwartz, J., and Koch, C. C. (2016). Magnetic alloys design using multi-objective optimization, Advanced Structured Materials series, Vol. 33, (eds.: Oechsner, A., da Silva, L.M., Altenbach, H.), Properties and Characterization of Modern Materials, 978-981-101601-1, 421721_1_En, (22), Springer, Germany.

[Journal Articles]

1. Fan, M., Liu, Y., Jha, R., Dulikravich, G. S., Schwartz, J., and Koch, C. C. (2016b). On the evolution of Cu-Ni-rich bridges of AINiCo alloys with tempering, Journal of Magnetism and Magnetic Materials, July 2016;

\section{DOl:10.1016/i.jmmm.2016.07.040.}

2. Fan, M., Liu, Y., Jha, R., Dulikravich, G. S., Schwartz, J., and Koch, C. C. (2016a). Effect of Cu-Ni-rich Bridges on the Microstructure and Magnetic Properties of Alnico Alloys, IEEE Transactions on Magnetism, August 2016, Vol. 52, No. 8, pp. 1-10; DOI: 10.1109/TMAG.2016.2555956.

3. Jha, R., Dulikravich, G. S., Chakraborti , N., Fan, M., Shwartz, J., Koch, C. C., Colaço, M. J., Poloni, C. and Egorov, I. N., (2016). Algorithms for design optimization of chemistry of hard magnetic alloys using experimental data, Journal 
of Alloys and Compounds, April 2016, DOI: 10.1016/j.jallcom.2016.04.218. Vol. 682, 2016, pp. 454-467.

4. Jha, R., Pettersson, F., Dulikravich, G. S., Saxen, H., and Chakraborti, N. (2015). Evolutionary design of nickel-based superalloys using data-driven genetic algorithms and related strategies, Materials and Manufacturing Processes, $30: 488-510$.

[Conference Proceedings]

1. Fan, M., Liu, Y., Jha, R., Dulikravich, G. S., Shwartz, J., and Koch, C. C. (2016b). Microscopic characterization of Cu-Ni-rich bridges in AINiCo alloys. 13th Joint MMM-Intermag Conference, San Diego, CA., USA, 11-15 January, 2016.

2. Jha, R., Dulikravich, G. S., and Colaço, M. J., (2015a) Design and optimization of magnetic alloys and nickel-based superalloys for high temperatures applications, COBEM-2015, paper 1284, Rio de Janeiro, Brazil, 6-11 December, 2015.

3. Jha, R., Dulikravich, G. S., Colaço, M. J., Egorov, I. N., Poloni, C., Chakraborti, N., Fan, M., Shwartz, J., and Koch, C. C. (2015b). Magnetic alloys design using multi-objective optimization. MS\&T 2015, Columbus, OH, USA, October 5-9, 2015.

4. Jha, R., Dulikravich, G. S., Colaço, M. J., Fan, M., Shwartz, J., and Koch, C. C., (2015c) Magnetic Alloys Design Using Multi-Objective Optimization, ACEX2015, Munich, Germany, June 29 - July 2, 2015.

5. Jha, R., Dulikravich, G. S., Chakraborti , N., Fan, M., Shwartz, J., and 
Koch, C. C., Colaço, M., J., Poloni, C., Egorov, I. N. (2015d) Algorithms for MultiObjective Design Optimization of Hard Magnetic Alloys Using Experimental Data, ICMM4, Berkeley, CA, USA, May 27-29, 2015.

6. Jha, R., Pettersson, F., Dulikravich, G. S., Saxen, H., and Chakraborti, N. (2014a), A combined experimental-computational approach to design optimization of high temperature alloys. In: ASME Symposium on Elevated Temperature Application of Materials for Fossil, Nuclear, and Petrochemical Industries, ASME, Seattle, WA, USA, March 25-27, 2014.

7. Jha, R., Dulikravich, G. S., Fan, M., Shwartz, J., Koch, C. C., Egorov, I. N., Poloni, C., (2014b) A Combined Computational-Experimental Approach to Design of High-Intensity Permanent Magnetic Alloys, CONEM2014, Uberlandia, Brazil, August 10-15, 2014. 


\section{ACKNOWLEDGMENTS}

I would like to express my sincere gratitude to my major advisor, Professor George S. Dulikravich for providing me with sufficient time and resources to understand the topic, his constructive comments while performing research and his help in presenting this work at several international conferences and publishing it in the form of journal articles and a book chapter.

I am grateful to my committee members Professor George S. Dulikravich, Professor Arvind Agarwal, Professor Surendra Saxena, Professor Sakhrat Khizroev and Professor Nirupam Chakraborti for allowing me to work under their esteemed guidance. I thank them for their cooperation in the form of constructive comments on my approach, providing me with recommendation letters and helping me in publishing journal papers and conference proceedings papers.

Thanks are also due to FIU MME graduate program co-ordinators, Professor Cesar Levy and Professor Yiding Cao, the MME chairperson, Professor Ibrahim Tansel and MME department staff Ms. Mabel Fernadez and Ms. Mariam Baruecco for helping me through document work so that I could fulfill various academic requirements on time.

I would like to thank our collaborators at North Carolina State University, namely Mr. Min Fan, Professor Justin Schwartz and Professor Carl C. Koch, for providing us with the experimental data used in this thesis.

Special gratitude to Professor Carlo Poloni, founder and president of ESTECO, for providing modeFRONTIER software free of charge, Professor Igor N. Egorov, CEO of Sigma Technologies, for providing IOSO software free of charge and 
Professors Marcelo J. Colaço and Souma Chowdhury for providing me with their input during this project.

I would like to thank Professor Henrik Saxen and Professor Frank Pettersson for inviting me to work in their lab at Abo Akademi, Finland in the summer of 2013.

I also want to thank my colleagues, Dr. Abas Abdoli, Mr. Diego Estumano, Mr. Sohail Reddy, Mr. Cesar Pacheco, Mr. Anthony Abrahao and Ms. Bruna Loiola. Interaction with them helped me in publishing several papers in international journals and conference proceeding on multi-disciplinary topics.

This work was funded by the US Air Force Office of Scientific Research under grant FA9550-12-1-0440 monitored by Dr. Ali Sayir. The views and conclusions contained herein are those of the authors and should not be interpreted as necessarily representing the official policies or endorsements, either expressed or implied, of the US Air Force Office of Scientific Research or the U.S. Government. The U.S. Government is authorized to reproduce and distribute reprints for government purposes notwithstanding any copyright notation thereon.

I received financial aid from FIU-UGS in the form of the Dissertation Year Fellowship and from FIU-GPSC for attending the MS\&T 2015 conference. I would also like to thank gratefully acknowledge AIST for providing me with financial aid for attending six technical workshops.

Finally, I would like to thank my parents, Mrs. Brinda Jha and Mr. Bindeshwar Jha for allowing me to realize my own potential and providing moral support during setbacks and continuous encouragement all through my life in realizing the goals that I set up for myself. 


\author{
ABSTRACT OF THE DISSERTATION \\ COMBINED COMPUTATIONAL-EXPERIMENTAL DESIGN OF HIGH \\ TEMPERATURE, HIGH-INTENSITY PERMANENT MAGNETIC ALLOYS WITH \\ MINIMAL ADDITION OF RARE-EARTH ELEMENTS
}

by

Rajesh Jha

Florida International University, 2016

Miami, Florida

Professor George S. Dulikravich, Major Professor

AINiCo magnets are known for high-temperature stability and superior corrosion resistance and have been widely used for various applications. Reported magnetic energy density $\left((\mathrm{BH})_{\max }\right)$ for these magnets is around 10 MGOe. Theoretical calculations show that $((\mathrm{BH}) \max )$ of 20 MGOe is achievable which will be helpful in covering the gap between AINiCo and Rare-Earth Elements (REE) based magnets. An extended family of AINiCo alloys was studied in this dissertation that consists of eight elements, and hence it is important to determine compositionproperty relationship between each of the alloying elements and their influence on the bulk properties.

In the present research, we proposed a novel approach to efficiently use a set of computational tools based on several concepts of artificial intelligence to address a complex problem of design and optimization of high temperature REE-free magnetic alloys. A multi-dimensional random number generation algorithm was used to generate the initial set of chemical concentrations. These alloys were then 
examined for phase equilibria and associated magnetic properties as a screening tool to form the initial set of alloy. These alloys were manufactured and tested for desired properties. These properties were fitted with a set of multi-dimensional response surfaces and the most accurate meta-models were chosen for prediction. These properties were simultaneously extremized by utilizing a set of multiobjective optimization algorithm. This provided a set of concentrations of each of the alloying elements for optimized properties. A few of the best predicted Paretooptimal alloy compositions were then manufactured and tested to evaluate the predicted properties. These alloys were then added to the existing data set and used to improve the accuracy of meta-models. The multi-objective optimizer then used the new meta-models to find a new set of improved Pareto-optimized chemical concentrations. This design cycle was repeated twelve times in this work. Several of these Pareto-optimized alloys outperformed most of the candidate alloys on most of the objectives. Unsupervised learning methods such as Principal Component Analysis (PCA) and Heirarchical Cluster Analysis (HCA) were used to discover various patterns within the dataset. This proves the efficacy of the combined meta-modeling and experimental approach in design optimization of magnetic alloys. 
CHAPTER

PAGE

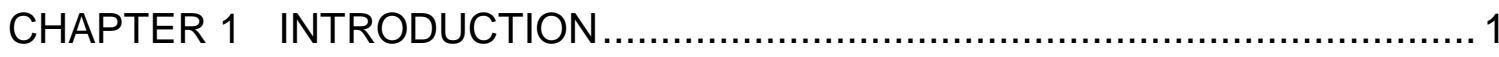

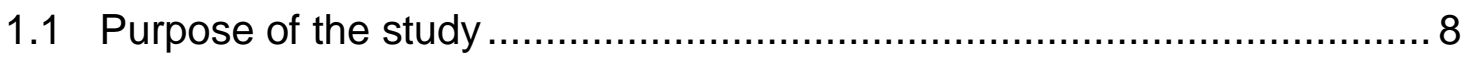

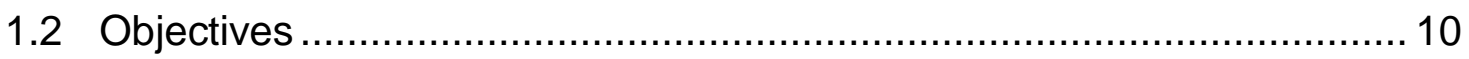

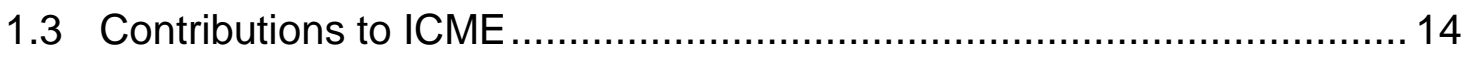

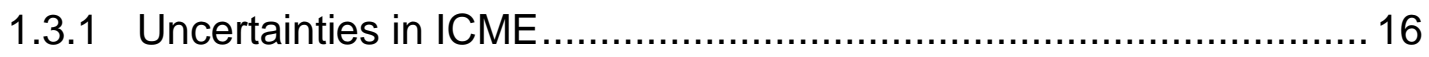

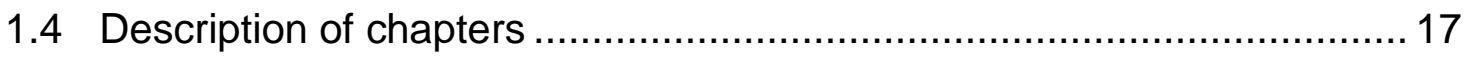

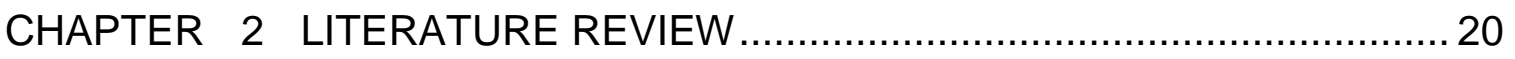

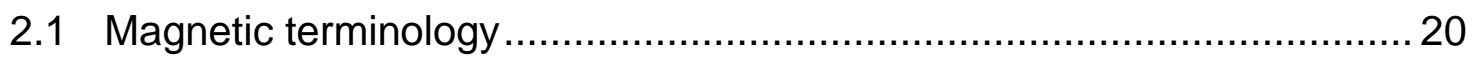

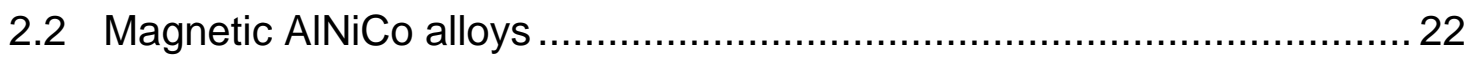

CHAPTER 3 RESEARCH PROBLEM AND METHODOLOGY ...................... 27

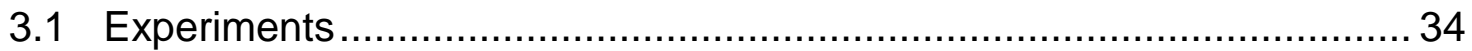

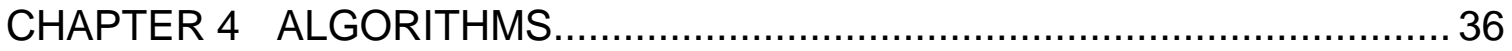

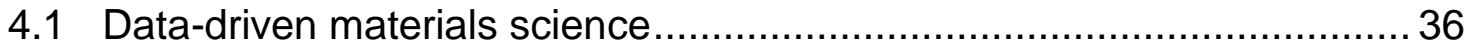

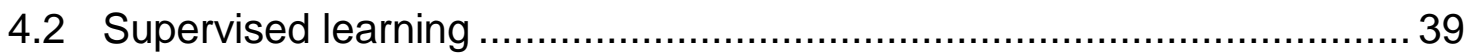

4.2.1 Surrogate Models/ Meta-Models ………........................................ 40

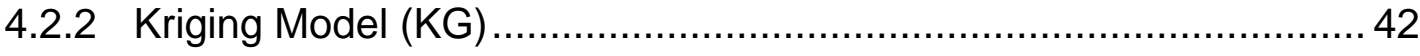

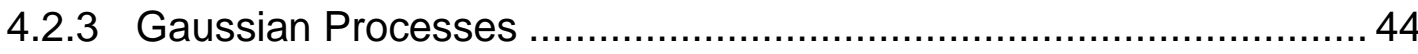

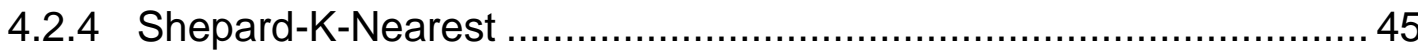

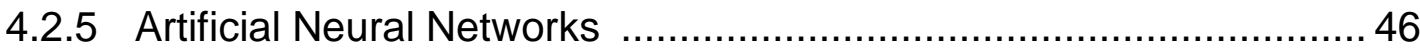

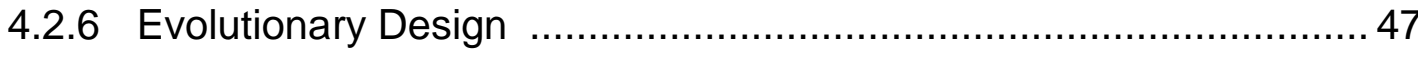

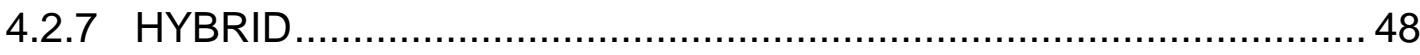

4.2.8 Performance measurements of a metamodel ............................... 49

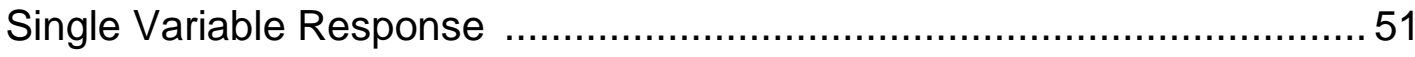

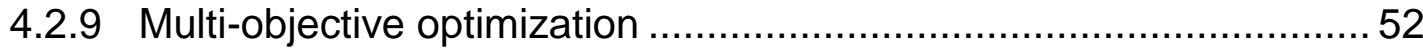

4.2.10 Evolutionary Algorithms for Multi-Objective Optimization ............... 55

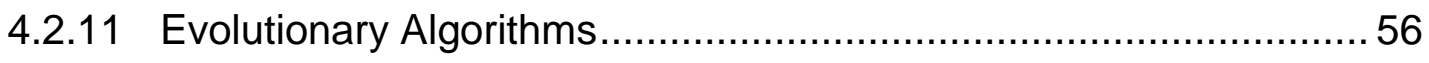

4.2.12 Non-Dominated Sorting Genetic Algorithm (NSGA-II) ................... 59

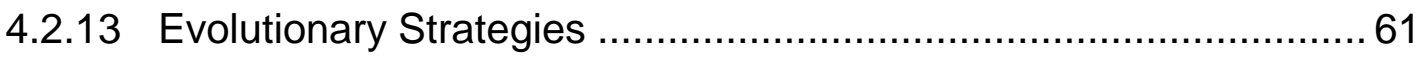




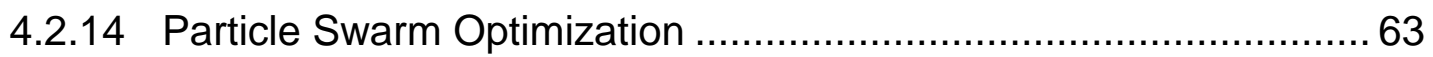

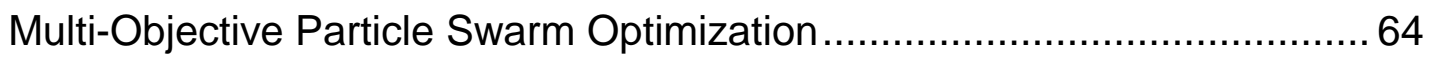

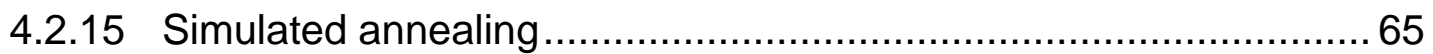

Multi-Objective Simulated Annealing (MOSA) ............................................ 66

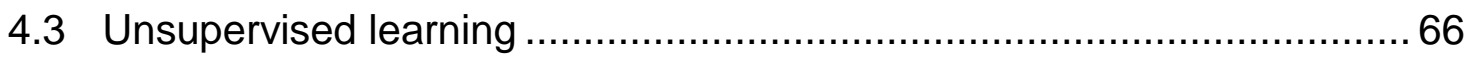

4.3.1 Clustering Analysis and related algorithms ..................................... 67

4.3.2 Principal Component Analysis (PCA) ............................................ 68

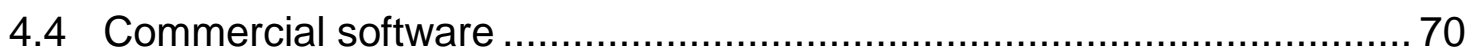

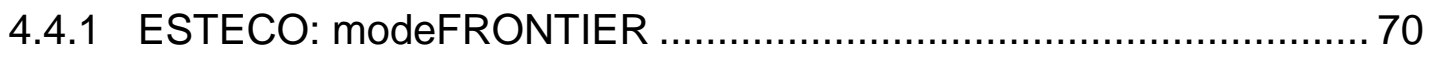

4.4.2 Indirect Optimization on the basis of Self-Organization (IOSO) ....... 70

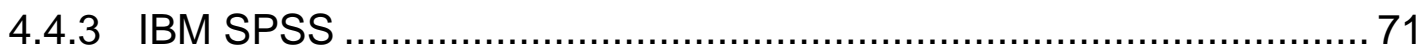

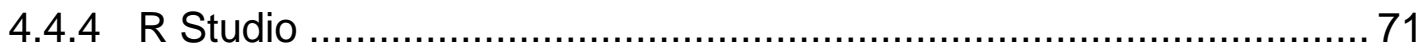

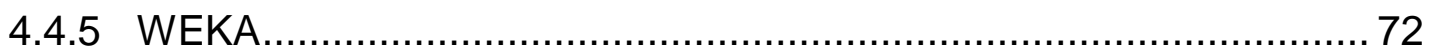

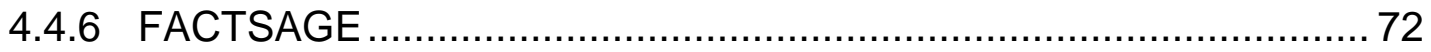

CHAPTER 5 RESULTS 1- SUPERVISED LEARNING …............................. 73

5.1 Results over the design cycles ……….......................................... 73

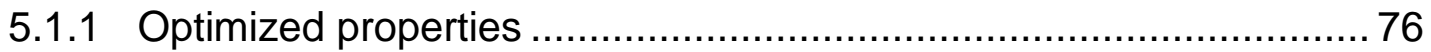

5.1.2 Alloy composition and distribution .......................................... 78

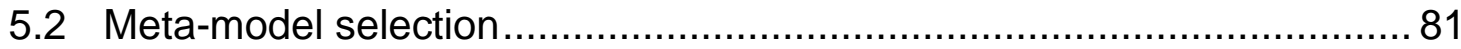

5.2.1 SVR analysis for selected models ............................................ 82

CHAPTER 6 RESULTS 2- UNSUPERVISED LEARNING ............................ 87

6.1 Heirarchichal Clustering Analysis (HCA) …...................................... 87

6.2 Principal Component Analysis (PCA) ............................................. 97

6.2.1 PCA for materials discovery .................................................... 108

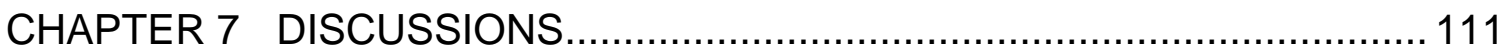

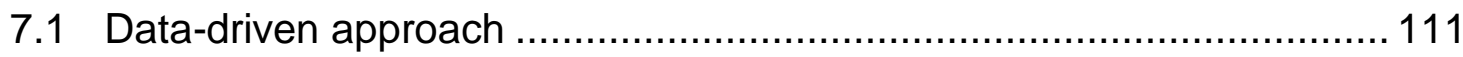

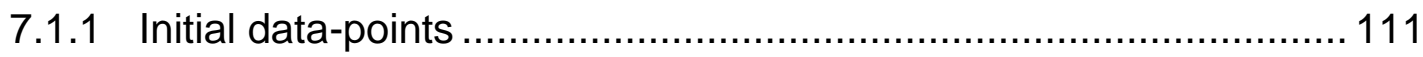

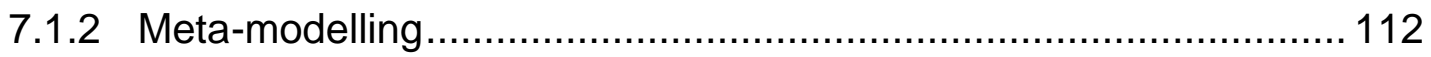

7.1.3 Multi-objective optimization ..................................................... 112

7.1.4 Unsupervised approach ......................................................... 114

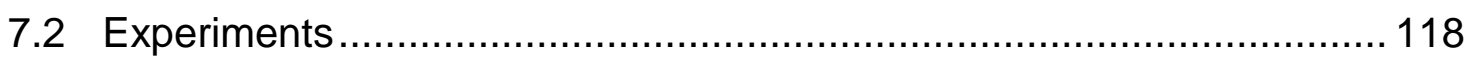


7.2.1 Characterization of alloy \# 95 .................................................. 118

7.2.2 Characterization of Cu-Ni rich bridges........................................ 123

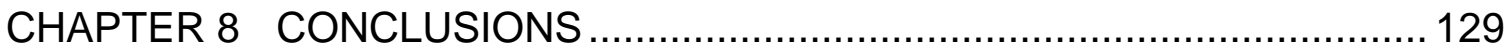

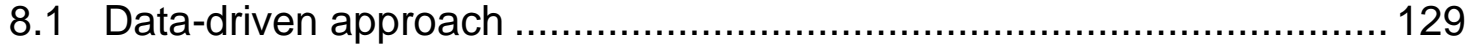

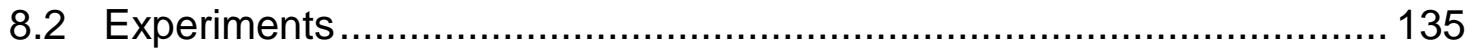

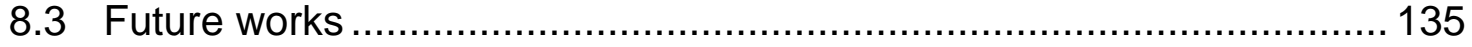

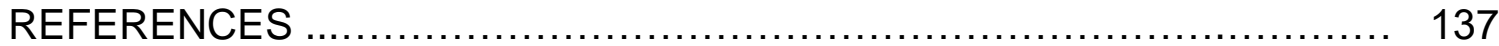

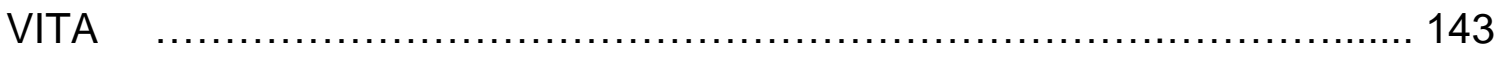




\section{LIST OF TABLES}

SL. NO.

PAGE

Table 1: $\quad$ Concentration bounds AINiCo type alloys ................. 28

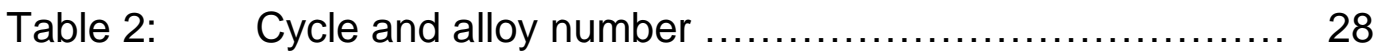

Table 3: Quantities to be simultaneously extremized using multiobjective optimization ................................. 29

Table 4: $\quad$ RBF approximation used in this work ................... 42

Table 5: $\quad$ Meta-model selected for targeted properties $\quad \ldots . . . . . . \quad 82$

Table 6: $\quad$ Single variable response for various properties ......... 83

Table 7: $\quad$ Clustering parameters in HCA analysis ............... 88

Table 8: $\quad$ Composition mapping of BSE image for alloy \#95 ........ 121

Table 9: $\quad$ EDS analysis of white spots observed in SEM 123 micrograph for alloy \#95

Table 11: $\quad$ Chemical composition(/properties) of alloy \#124 ........ 131

Table 12: Evaluating scope of REE (Cerium) addition............. 136 


\section{TABLE OF FIGURES}

SL. NO.

PAGE

Figure 1: $(B H)_{\max }$ vs temperature for various magnetic systems, (Kramer et al., 2012)

Figure 2: Rare-earth element, global deposits (Humpheries, 2013) 5

Figure 3: $\quad(B H)_{\max }$ vs cost, (Kramer et al., 2012) $\ldots \ldots \ldots \ldots \ldots \ldots \ldots \ldots \ldots$

Figure 4: B-H curve: shows relation between $H_{c}, B_{r}$ and $(B H)_{\max }$ . (Kramer et al., 2012)

Figure 5: Magnetic energy density vs magnetic coercivity

Figure 6: Magnetic energy density vs magnetic remanence, comparison of solutions by various approaches

Figure 7: Magnetic coercivity vs magnetic remanence, comparison of solutions by various approaches ......

Figure 8: Scatter: Distribution of $\mathrm{Fe}$ and $\mathrm{Co}$ in variable space, comparison of solutions by various approaches .........

Figure 9: Scatter: Distribution of $\mathrm{Fe}$ and $\mathrm{Cu}$ in variable space, comparison of solutions by various approaches..............

Figure 10: Scatter: Distribution of $\mathrm{Ni}$ and $\mathrm{Al}$ in variable space, comparison of solutions by various approaches

Figure 11: Scatter: Distribution of $\mathrm{Hf}$ and $\mathrm{Cu}$ in variable space, comparison of solutions by various approaches

Figure 12: SVR: Nickel on magnetic energy density $(B H)_{\max } \ldots \ldots$

Figure 13: SVR: Nickel on magnetic Coercivity $\left(H_{c}\right) \ldots \ldots \ldots \ldots \ldots . . . . \quad 85$

Figure 14: SVR: Nickel on magnetic remannence $\left(B_{r}\right) \ldots \ldots \ldots \ldots . \quad 85$

Figure 15 SVR: Nickel on saturation magnetization $\left(M_{s}\right) \ldots \ldots \ldots$ 
Figure 16: Dedrogram plot from HCA $\quad \ldots \ldots \ldots \ldots \ldots \ldots \ldots \ldots \ldots \ldots$

Figure 17: Simplified dedrogram plot from HCA .......... 88

Figure 18: Clusters scatter: $(B H)_{\max }$ vs Aluminum ............... 89

Figure 19: Cluster scatter: $H_{c}$ vs Copper ....................... 90

Figure 20: Cluster scatter: $B_{r}$ vs Copper ........................ 91

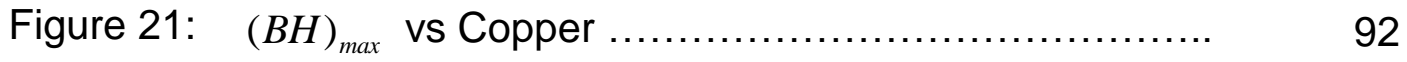

Figure 22: $\quad H_{c}$ vs Titanium ................................. 93

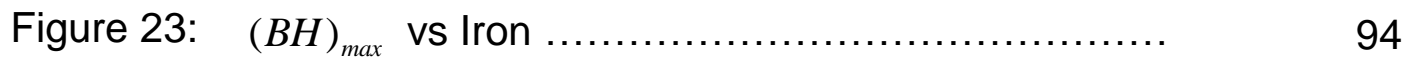

Figure 24: Clusters scatter: $(B H)_{\max }$ vs. Nickel ................. 95

Figure 25: Clusters scatter: $H_{c}$ vs. Niobium .................... 96

Figure 26: Clusters scatter: $B_{r}$ vs. Niobium ................... 96

Figure 27: Clusters scatter: $(B H)_{\max }$ vs. Niobium ................ 97

Figure 28: Scree plot for PCA analysis: 2 PCA components were

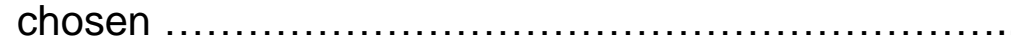

Figure 29: Orientation of various properties in the PC space ......... 100

Figure 30: Scree plot for PCA analysis: 3 PCA components were chosen

Figure 31: Orientation of various elements in the PC space ......... 101

Figure 32: Scree plot for PCA analysis: 3 PCA components were chosen ..................................................

Figure 33: Orientation of various properties in the PC space ......... 102

Figure 34: Scree plot for PCA analysis: 3 PCA components were 103 
chosen

Figure 35: Orientation of various elements in the PC space

Figure 36: Scree plot for PCA analysis: 3 PCA components were chosen

Figure 37: Orientation of various properties in the PC space ......

Figure 38: Scree plot for PCA analysis: 3 PCA components were chosen

Figure 39: Orientation of various elements in the PC space

Figure 40: Scree plot for PCA analysis: 2 PCA components were chosen

Figure 41: Orientation of various properties in the PC space

Figure 42: Orientation of various elements in the PC space ........ 109

Figure 43: Orientation of various elements in the PC space .......... 110

Figure 44: Phase distribution diagrams for alloy (a) 84, (b) 86, (c) 124 and $(d) 126$

Figure 45: Phase distribution diagram obtained after modifying the composition of Alloy \#124

Figure 46: Optical micrograph for alloy \# 95 showing white spots ....

Figure 47: Back scattered image used for compositional mapping of alloy \# 95 viewings along the transverse orientation (parallel to the magnetic field)

Figure 48: SEM image showing the white spots to be be analyzed by EDS for alloy \# 95 viewings along the transverse orientation (parallel to the magnetic field)

Figure 49: HAADF images along [001], and corresponding EDS maps for Sample A with Titanium

Figure 50: HAADF images along [001], and corresponding EDS maps for Sample B without Titanium 
Figure 51: High-resolution HAADF image and EDS scan for sample A with Titanium (a) HAADF image, (b) EDS scan from $\alpha_{1}$ to $\alpha_{2}$ phase, (c) EDS scan at higher resolution. (Fan et al., 2016b)

Figure 52: Scatter plot of 180 alloys on the second quadrant of B-H curve 


\section{ABBREVIATIONS}

AKR Anisotropic Kriging

BCC Body Centered Cubic

BioGP Bi-Objective Genetic Programming

CALPHAD Calculation of phase diagram

ED Evolutionary Design

EDS Energy-dispersive x-ray spectroscopy analysis.

EvoNN Evolutionary Neural Network

FAST FAST optimizer in modeFRONTIER

FCC Face Centered Cubic cell

GP Gaussian Processes

HAADF High-angle Annular Dark Field

HCA Hierarchical Cluster Analysis

IMQ Inverse Multi-Quadrics

ICME Integrated Computational Materials Engineering

IOSO Indirect Optimization based on Self-Organization algorithm

KR Kriging

MCDM Multi-Criterion decision making

MOPSO Multi-Objective Particle Swarm Optimization

MOSA Multi-Objective Simulated Annealing

MQ Multi-Quadrics

NSGA2 Non-Dominated Sorting Genetic Algorithm 2

PCA Principal Component Analysis 
RBF Radial Basis Function

REE Rare Earth Element

ROMEO Replacement and Original Magnet Engineering Options

SQUID Quantum Design superconducting quantum interference device magnetometer

TEM Transmission electron microscope . 


\section{NOMENCLATURE}

$\alpha$ High temperature B2 single phase

$\alpha_{1}$ Iron-cobalt rich ferromagnetic phase (B2

$\alpha_{2} \mathrm{Ni}-\mathrm{Al}$ rich phase (L21)

$\gamma$ FCC phase

$B_{r}$ Magnetic remanence ( Tesla)

$H_{c}$ Magnetic Coercivity (Oersted)

$(B H)_{\max }$ Magnetic energy density

$M_{s}$ Saturation magnetization

$M_{r}$ Remanence magnetization

$\mu$ Magnetic permeability

$j H_{c}$ Intrinsic coercive field

$\rho$ Density 


\section{CHAPTER 1 INTRODUCTION}

In this era of economic development, one of the major challenges is to deal with preservation of the environment. Stringent norms regarding various emissions imposed in the developed economies have made it difficult for industries to compete with rivals globally and operate profitably. As a result, many industries are being relocated at offshore locations (mainly in developing countries) where the environmental norms are not stringent. In the past few decades, this has severely affected the US economy and because of this, US has lost its supremacy in global production. Magnetic materials market has been estimated at USD 55.52 billion in 2014 and by 2020 it is expected to be worth USD 96 Billion (M\&M, 2016). Hence, investment in research in magnetic materials and its accelerated implementation is highly desired. This will be helpful in consolidating the position of the US as a global leader in production of magnetic materials. Regarding emissions, especially emissions from vehicles (car, trucks, motorbikes, etc.) is important as it is among one of the major sources. In recent years, there has been significant research in finding ways to address this problem by going for alternative fuels. One of the major aim is to come up with vehicle designs that will be efficient enough to have a fuel efficiency of $54.5 \mathrm{mpg}$ or more (EPA, 2012).

Alternative fuels such as bio-fuels will be helpful in reducing greenhouse gas emissions when compared to fuels generally used in internal combustion engines. Another alternative source of energy is by generating required power from electric motors. 
Permanent magnet-based synchronous generators are almost emission free. Regarding performance for application in wind turbine engines, these generators were found to be competitive with induction generators. Induction motors are quite heavy and require regular maintenance cycles, which is expensive, and hence add up to the total cost. Recently, a number of hybrid generators have replaced traditional induction motors. Hybrid generators use both, a traditional induction generator and a permanent magnet generator, thus lowering the cost of maintenance. Hybrid motors use one-third of the weight of permanent magnets usually used in permanent magnet generators. One of the drawbacks of induction generators is that they suffer from gearbox failures. In hybrid generators, this is further mitigated, while in permanent magnet generators, these failures can be eliminated. Use of magnets in generators has its constraints regarding the size and weight of the magnet used. Hence, the magnets to be used must meet the design requirements, especially in hybrid generators/vehicles where there is limited space between the internal combustion engine and wheel wells. Apart from the shape and size constraints, these magnets must be dense enough to generate power in order to meet the requirements of the vehicle. Over the years, there has been significant research in finding ways to work on improvement of the properties of these magnets and it has resulted in discovery as well as improvement of REEbased magnets.

REE-based magnets have a very high magnetic energy density $\left((B H)_{\max }\right)$. This means that it is possible to synthesize smaller magnets, while maintaining the superior magnetic properties. These magnets also have a higher coercivity $\left(H_{c}\right)$, 
making it difficult to demagnetize under external magnetic fields. These magnets have higher remanence $\left(B_{r}\right)$. $\mathrm{Br}$ corresponds to the amount of magnetic flux density left in the magnet after removal of external magnetic field. Neodymiumbased magnets $\left(\mathrm{Nd}_{2} \mathrm{Fe}_{14} \mathrm{~B}\right)$ are the strongest available magnets in this family. However, Nd-Fe-B (Neodymium-Iron-Boron) performs the best up to 150 degree centigrade. Figure 1 shows the plot between $(B H)_{\max }$ and temperature for various systems of magnets currently in application (Kramer et al., 2012), (Jha et al., 2016). In order to improve upon this, Dysporium (Dy) is added. Dysporium slightly increases Curie temperature and most importantly, it significantly increases the resistance to demagnetization up to about $200^{\circ} \mathrm{C}$. This improvement in hightemperature stability due to Dy addition is achieved by compromising marginally on magnetic energy density. Dysporium is a heavy element and expensive, too. Dysporium content in $\mathrm{Nd}$-based magnets can go up to 12 percent for applications at about $220^{\circ} \mathrm{C}$, but then synthesis of this resulting magnet will not be profitable at the current prices. Researchers around the globe are working on reducing Dy content in Nd-based magnets. From $150^{\circ} \mathrm{C}$ to $350^{\circ} \mathrm{C}$, Sm-Co (Samarium-Cobalt) magnets are used. These magnets usually need a protective coating in order to prevent corrosion. REE-based magnetic materials are essential in electric cars, in wind turbine electric generators, and any high-efficiency electric devices requiring magnetic fields. Hence, REEs are classified as strategic materials, determining which national economies will hold out and thrive in the post-combustion-engine era. 
Deposits of most of the rare earth elements used for synthesizing these magnets are located in China and the Russian federation (as shown in Figure 1). Due to depleting resources and stringent trade rules from the suppliers, it is important to look at other options to synthesize these magnets (Mcguiness et al., 2015). Due to these restrictions, the cost for REE has fluctuated a lot over the past few years. This makes REE based magnets the most expensive magnets among all the grades currently in application (Figure 3).

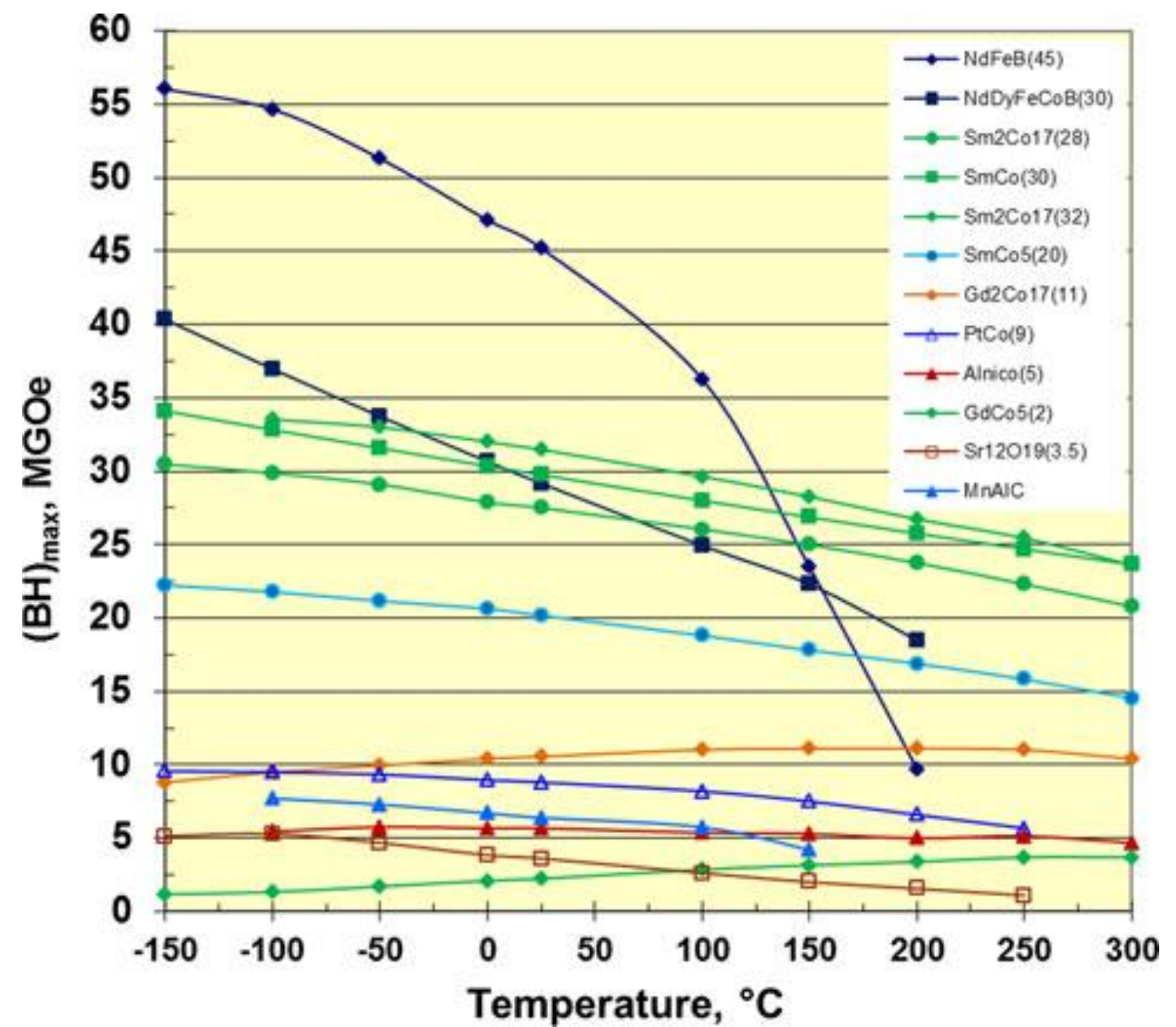

Figure 1: $(B H)_{\max }$ vs temperature for various magnetic systems, (Kramer et al., 2012) 


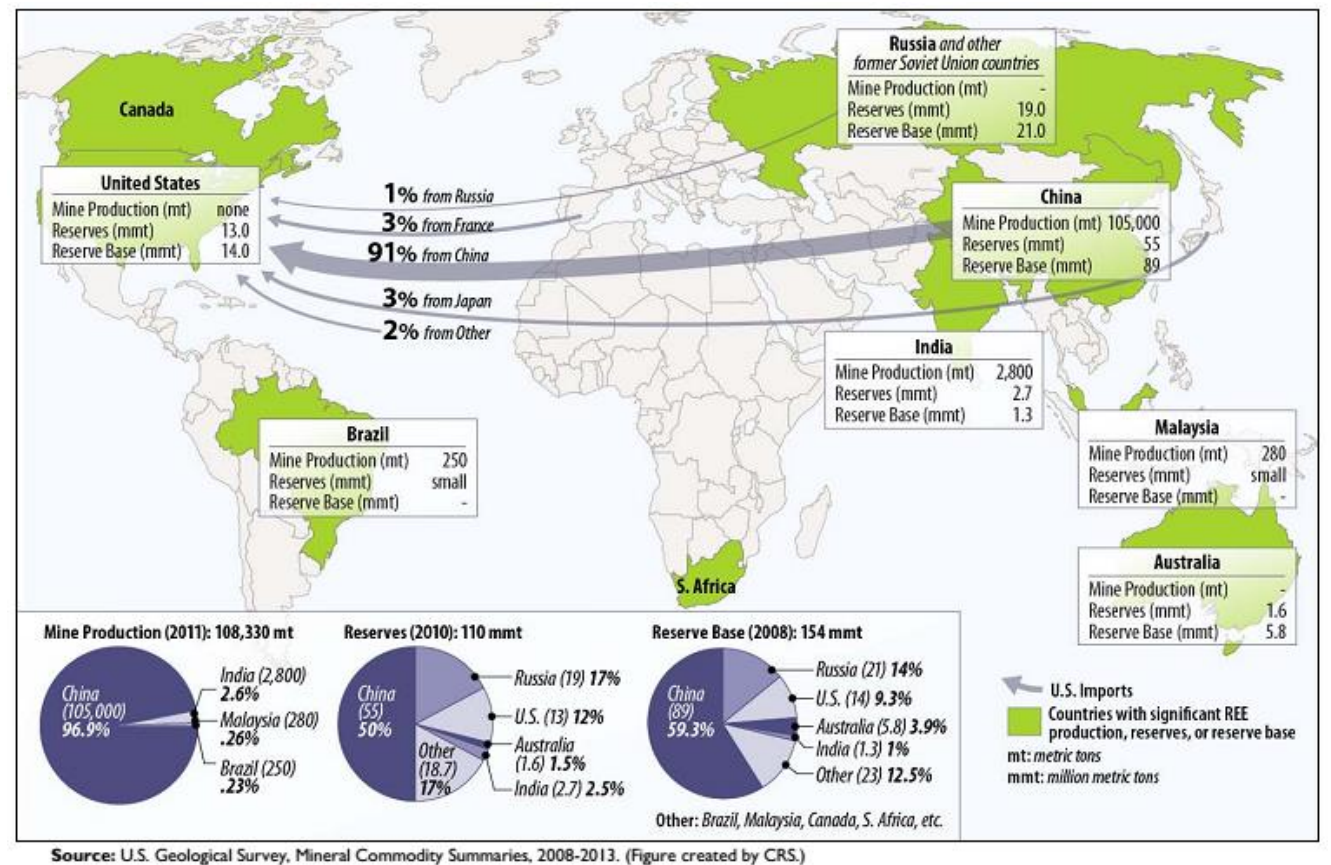

Figure 2: Rare-earth element, global deposits (Humpheries, 2013)

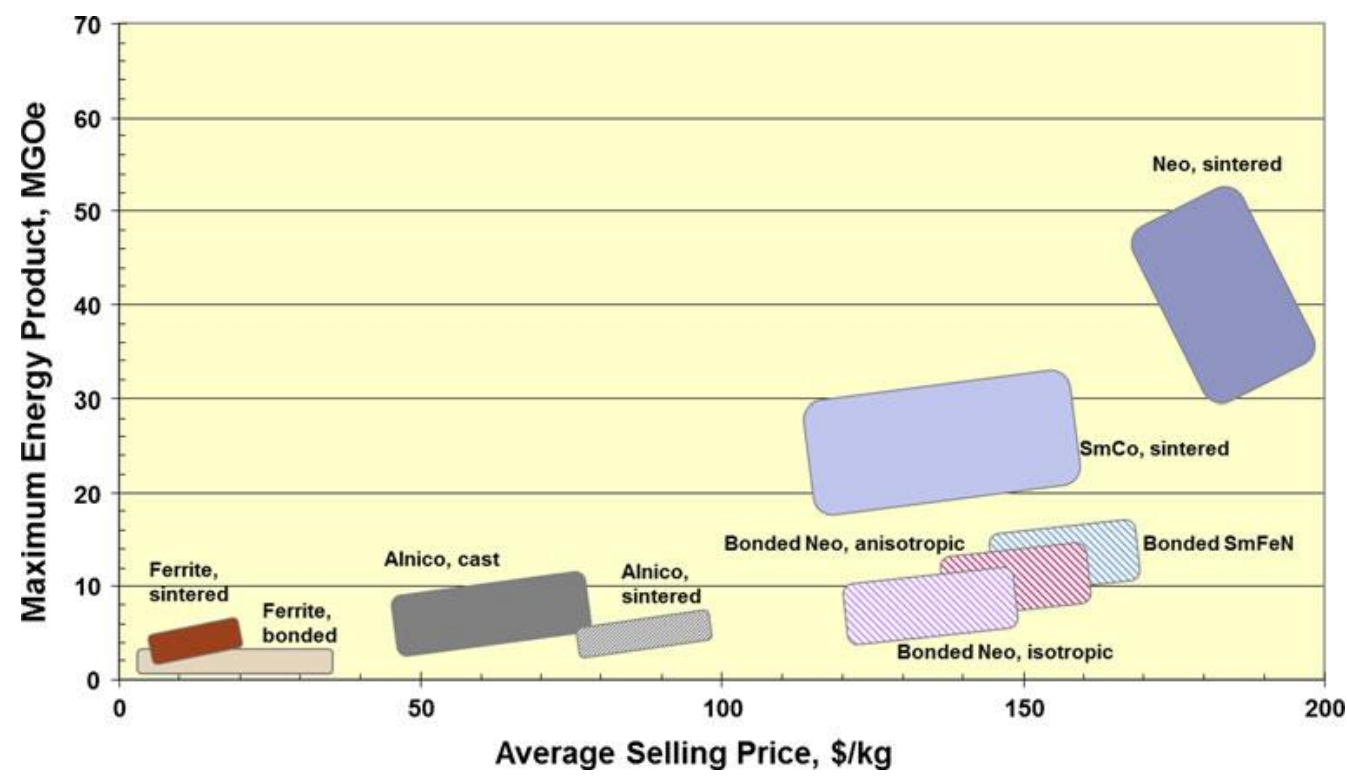

Figure 3: $(B H)_{\max }$ vs cost, (Kramer et al., 2014) 
On one hand, these restrictions have severely affected various industries due to increasing demand in high strength magnets, while on the other side, this has proved to be an opportunity for researchers around the globe to come together. Leading research labs in the US and Europe have formed collaborations to look for alternatives and work towards accelerated implementation. Replacement and Original Magnet Engineering Options, or ROMEO (Mcguiness et al. 2015), based in Europe suggested a few recommendations in order to address this problem and for accelerated implementation of these magnets. These suggestions can be listed as follows:

1. Recycling of devices that contain rare-earth elements.

2. Search for new mines with REE deposits or look for viable options in order to start mining at mines that were closed as they were unable to mine profitably in the past.

3. Development of high energy magnets that use minimal or no rare earth elements.

There has been significant progress in recycling of rare-earth elements. Researchers have been able to separate Neodymium from Dysprosium. This is an important innovation and these elements can now be extracted from loudspeakers, headphones and even wind turbines. Regarding mining, efforts are being made in the US to revive the mine at Molycorp as it was shut down due to environmental regulations as well as a significant drop in revenues due to lower rare-earth prices from China (Cen-ACS, 2015). Even then, working on (1) and (2) will take time and one will have to still be dependent on suppliers in the near future. Hence, (3) 
development of high-energy magnets that use minimal or no REE, seems to be a viable option.

In order to proceed further, researchers around the globe attempted to reexamine AINiCo magnets by experimentation, characterization and computational modelling. Although there has been limited research on these magnets in the since late 1970's, commercial production of these magnets never stopped. Hence, it is better to use advanced tools to re-examine AINiCo magnets for development and accelerated implementation of rare-earth free magnets. Ames lab in the US is another center that has been extensively working on these type of magnets and has demonstrated significant scope of improvement in these magnets.

AlNiCo magnets (Cullity and Graham, 2009) are permanent magnetic alloys based on the Fe-Co-Ni-Al system without REEs. AlNiCo magnets have high $\mathrm{Br}$ values, compared to REE magnets. These magnets have lower Hc values and can be demagnetized in the presence of an external magnetic field. These magnets can be easily magnetized to saturation. These magnets can be cast into complex shapes while magnetizing it in the production heat treatment stages. These magnets possess excellent corrosion resistance and high-temperature stability. These are the only magnets that are stable up to $800^{\circ} \mathrm{C}$ (Curie temperature). Above-mentioned properties have been successfully exploited by researchers in the past and these magnets are a perfect choice for military and automotive sensor applications.

Most of the research on AINiCo alloys dates back to the end of the 1970's (since the development of REE based magnets). Currently, a commercial AINiCo 
composition can consist of eight or more elements. Much of the characterization work deal with nanoscale features, but there is limited information on the effect of these alloying elements on various targeted properties. Apart from that, the alloy is subjected to a complex thermo-magnetic treatment, that if improved may help in achieving superior properties. In recent years, use of advanced characterization tools along with high-throughput experiments and computer simulations have helped the researchers to re-examine AINiCo alloys to work upon its improvements. Skomski and his coworkers (Skomski et al., 2013) demonstrated that the theoretical magnetic energy density that can be achieved for these alloys is in excess of what has been achieved for best grades of commercially available AINiCos (AINiCo 5-9). A targeted magnetic energy density of about 20 MGOe at about $180^{\circ} \mathrm{C}$ will be helpful in covering the gap between the magnetic properties achieved by AINiCo and REE based magnets (Figure 1-1 and Figure 3). If we consider the cost, then this AINiCo will be able to compete with REE based magnets for quite a few important energy conversion issues.

\subsection{Purpose of the study}

There has been a sharp increase in price of rare-earth elements for magnets and it is fueled by a sharp increase in the demand of high end electronics, storage devices, guided systems for defense to name a few. At the same time, the industry has been dealing with suppliers that are not reliable mainly due to the locations of the mines and the political relationship with these countries. It is important for researchers to look for alternative options to full fill the demand of various industries, While for a few applications we do not have any other option 
other than REE-based magnets due to their high energy density. For these applications, we have to still rely on interrupted supply or go for recycling until we can find a reliable supplier. However, for quite a few other applications, one can use magnets that covers the gap between rare-earth and AINiCo magnets. Here, one can focus on improving the properties of the commercial magnets by adjusting their chemistry and modifying their standard manufacture protocol. At the same time, attempts are being made to discover new alloys that can be competitive enough to replace rare-earth magnets in a few energy conversion applications. To accelerate this process, one needs to think out of the box and proceed towards using computational tools to aid conventional experimentation in making minor adjustments. Hence, the alloy design space needs to be explored further and it is not possible to do with random experimentation. In recent years, there has been a rise in the use of computational tools in materials modelling. Theoretical calculations suggest that there is scope of improvement in the achievable properties of these magnets by either adjusting its composition or by modifying the thermo-magnetic treatment to which these alloys are exposed. This work is aimed at determining composition-property relationship for these magnets that will help in developing a knowledge-base for improvement of new alloys and can be used as a foundation for development of new alloys for targeted properties. Another important aspect of this work will be to eliminate a few elements that are found lest influential and make way for REE additions.

In the present research work, a novel approach is presented for creating a work plan for efficiently utilizing existing computational tools for design and multi- 
objective optimization of permanent magnetic alloys that is supported by experimentation. The proposed research combines a number of numerical design optimization algorithms with several concepts from artificial intelligence and experimentally evaluated desired properties of an affordable set of candidate alloys. Various statistical tools further screened these alloys in order to determine any specific trend in the dataset that can be supported by literature. This information will be helpful for the research community in developing a material knowledge base for the design of new alloys for targeted properties.

Resultant magnets are expected to have high temperature stability as these belong to the class of AINiCo alloys at the same time we expect to achieve superior properties at par with those demonstrated by various researchers by theoretical calculations. Thereafter, we worked upon modifying the thermo-magnetic treatment protocol for improved results.

\subsection{Objectives}

In recent years, a significant amount of research has been reported in designing rare-earth free magnets. These works includes and are not limited to abinitio calculations, theoretical modeling as well as experimental modeling. In our work, we focused on some of the critical aspects that have not been addressed or can be addressed in a different way to accelerate the alloy development and accelerated implementation of these alloys.

Our effort can be listed as follows:

1. Selection of an initial set of alloys: We used one of the best-known random number generator to generate this set. Usually, it is done by hit and trial. 
Hence, it may be possible that the experimentalist will miss the set of combination that can be the best for a certain thermo-magnetic protocol followed by them.

2. Theoretical modeling: We used a thermodynamic database to check upon the stability of critical phases. This was done for screening the initial set of alloys. This will help the experimentalist in designing the thermo-magnetic protocol for a particular set of combination and to avoid temperature regimes that may lead to formation of phases that may negatively affect the magnetic properties. We also used limited information from databases that provide results from ab-initio based calculations to support our proposal.

3. Experimental modeling: These alloys were manufactured and tested for checking upon improvement in properties.

4. Meta-modeling: AINiCo alloys in our present research consist of eight elements. Calphad and ab-initio calculations can handle only alloys with 3-4 alloying elements. In order to address this, we developed meta-models to link composition of elements with the bulk properties. We used several concepts of artificial intelligence to develop meta-models. We checked our meta-models developed in the previous sections, to test their capabilities in determining composition-property relationship. We found that our meta-models were able to mimic the limited knowledge we have from the literature for a few elements and associated properties. As expected from a noisy data set, we got many mixed results too. Meta-models that performed the best on various accuracy measures were selected for future use. 
5. Multi-objective optimization: In alloy developments, we usually face conflicting objectives. Thus, random experimentation can prove to be misleading. In this work, we used several concepts of evolutionary algorithms for multiobjective optimization of targeted properties. Due to optimizer limitations, we restricted the number of objectives to be optimized at three at a time. We will see in the literature review that, $(B H)_{\max }$ is the area of the largest rectangle that can be inscribed in the second quadrant of the $\mathrm{B}-\mathrm{H}$ curve. Thus, in order to maximize $(B H)_{\max }$ we need to maximize both $B_{r}$ and $H_{c}$. However, $B_{r}$ and $H_{c}$ are conflicting. Here, we are dealing with a problem where one of the objective depends on two other objectives that are conflicting in nature. In addition, we have to attempt to maximize all three of them. Hence, multi-objective-optimization is best suited for this type of problem.

6. Multi-Criterion Decision Making (MCDM): As mentioned, 3 objectives were simultaneously optimized at a time due to optimizers limitations, but several other objectives/properties are also important for implementation of magnets. Additionally, optimization results yield thousands of new sets of combinations. Manufacturing all of these is not feasible and not economical. If we select a few data points at random, then the whole purpose of using meta-modeling and multi-objective optimization will be meaningless, as we could have generated a new combination by using a random number generator (as we did in the beginning when we had limited knowledge of the system). At this point, it is time for the expert to look into the problem. Here, it was done by using a popular statistical algorithm, known as MCDM. In optimization problems, there is no unique 
solution, thus it is better to know the experts preference based on his expertise and specific needs. In MCDM, the expert can specify his needs and based on their understanding, theoretical knowledge and optimization results, can select a few alloys for manufacture by using MCDM. This will save time and this method is quite popular in the research domain. Hence, our suggestions have a strong statistical basis and can be accepted by the materials research community.

7. Sensitivity analysis: It was performed in order to look for correlations and to discover patterns or trends in the dataset. Initially the dataset was analyzed using Pearson's linear correlation. Since, the dataset is quite noisy, linear correlations were quite low. It was expected, and we are dealing with a multicomponent system, where even a small amount of undesirable elements has the potential to shift the equilibrium and one can expect a completely different property. In order to deal with this, we used several machine-learning algorithms that has been successfully implemented in computational materials science domain. We used Principal component analysis (PCA) and Hierarchical clustering analysis (HCA) to find meaningful information from the dataset. Clustering analysis was performed to divide the data set into disjoint groups and look for specific patterns in each group and the whole dataset. In this way, we can eliminate a certain data point or a cluster that we think is not contributing in our analysis based on our expert knowledge (both computational expert as well as a metallurgist).

8. Experimental modelling: It was performed at NCSU. Peculiar findings from this work has been listed in section 7.2. 


\subsection{Contributions to ICME}

At present, researchers around the globe are working on finding alternatives in order to design magnetic alloys that will be able to cover the gap between the properties achieved by AINiCo magnets and the rare-earth magnets. An initiative in Europe, Re- placement and Original Magnet Engineering Options, popularly known as ROMEO has laid down certain guidelines for researchers that will help to address this topic (Mcguiness et al., 2015). It varies from recycling devices containing rare-earth metals to finding new mines outside China and Russian federation as well as designing magnets without rare-earth additions or with a minimal amount of those rare-earth elements that are less critical in the sense of supply (Ronning and Bader, 2014), (Kramer et al., 2012). This will help in addressing a few important energy conversion applications. Sellmayer and his coworkers (Sellmyer et al., 2013) worked on a few rare-earth free alloys and the properties were found to be in the vicinity of AINiCo alloys. Zhou and his coworkers (Zhou et al., 2014) demonstrated the scope of improvement of magnetic properties of AINiCo alloys by theoretical modeling. However, the difference between the theoretically calculated and the measured properties were quite large for $(B H)_{\max }$ and $H_{c}$. Hence, random experimentation may be misleading in terms of improvement in alloy properties while being both expensive and time-consuming.

Advances in multiscale-materials modeling can be subdivided into three categories mentioned below:

1. Historical: Serial paradigm

2. Current: Integrated Computational Materials Engineering (ICME) 
3. Future: Virtual materials design.

Phase 2 and 3 demands for integration of microstructure, properties, numerical codes, experimental methods, etc. In the past, the research community has focused on developing computational tools to establish a relationship between micro-structure and desired properties of the alloy. This led to the development of the CALPHAD (Calculation of Phase Diagram) approach in the 1960's. In the 1970's till the mid 1980's, the computational materials science (CMS) established itself as a separate discipline of its own. At present, the ICME approach aims to combine the previous findings in order to aid experimentalists in developing new alloys with advanced properties.

Designing a new alloy system is a challenging task mainly due to a limited experimental database. In order to develop a reliable knowledge base (Rajan, 2013) for the design of new alloys, one needs to focus on determining various correlations (composition-property, property-property, and compositioncomposition) from the available databases (simulated and experimental). This information can be coupled with the theoretical knowledge (atomistic and continuum based theories) to develop the knowledge base. Integrated Computational Materials Engineering (ICME) approach (Horstemeyer, 2012) and materials genome initiative highlighted the importance and growing application of computational tools in the design of new alloys. In recent years, various datadriven techniques combined with evolutionary approaches (Egorov-Yegorov and Dulikravich, 2005) have been successfully implemented in alloy design (Egorov- 
Yegorov and Dulikravich, 2005), (Jha et al., 2015b), (Jha et al., 2016), and in improving thermodynamic databases such as ThermoCalc (Guide and Version, 2002), (Thermocalc, 2015) for alloy development. Jha et al. (2015b) demonstrated the scope of use of these databases in designing Ni-based superalloy and (Rettig et al., 2015) performed a few experiments to confirm his findings. Data mining approaches such as Principal Component Analysis (PCA) and Partial Least Square (PLS) regression have been successfully used in designing new alloys (Toda-Caraballo and Rivera-Diaz-Del-Castillo, 2015), (Settouti and Aourag, 2015). Additionally, various machine-learning algorithms have been used to address a vast range of problems in materials, design (Mueller et al., 2015). These applications demonstrate the efficacy of application of computational tools for materials design.

\subsubsection{Uncertainties in ICME}

One of the key challenges in ICME is dealing with uncertainty. Uncertainty in ICME can be summarized as below (Panchal et al., 2013):

1. Uncertainty: Identification and quantification of sources and develop mathematical representation

(a) Aleatory or irreducible uncertainty: Randomness of materials Represented by probability distribution Can be addressed by probability theory

(b) Epistemic or reducible uncertainty: Lack of knowledge due to idealization, approximation, numerical errors Bayes probability theory used by others, but limited success Alternate fuzzy set theory, possibility theory 
2. Uncertainty propagation or uncertainty analysis:

(a) From input of one model to its output

(b) From lower level models to higher level models

(c) From materials composition to structure

(d) Bayesian approaches can be used

3. Uncertainty mitigation: Reducing the effect of uncertainty in materials design

(a) Multidisciplinary design and optimization (MDO)

(b) Surrogate modeling and statistical analysis

4. Uncertainty management: Decision on the appropriate level of uncertainty based on the tradeoff between effort and benefit

In this work, we have made an effort to address (3) and (4), that is Uncertainty propagation and Uncertainty mitigation.

\subsection{Description of chapters}

In the following section, there is a brief introduction to the contents of the various chapters. This part is added with the introduction so that a reader interested in a certain section or seeking a specific information can directly go to that chapter without any loss of continuity. However, all the chapters are interconnected, so it is recommended for a reader to go through the full thesis for better understanding.

Chapter 2 Literature Review: This chapter deals with the basic physics of the problem. Various terms associated with magnets has been introduced. Information related to composition property relationship is quite important for our 
work. Each element and the way it affects the bulk magnetic properties has been reported from the available literature. This information proved to be helpful in the later stages, that is during development and selection of meta-models and alloy selection by MCDM.

Chapter 3 Research problem and methodology: In this chapter, we stated the research problem and our approach to solve the problem. This chapter will be helpful for researchers in the future, as it provides basic guidelines on how to attempt a complex task of alloy development for targeted properties from the scratch and limited knowledge of the system. Experiments were carried out at North Carolina State University so we have added a brief account on manufacture protocol followed by them.

Chapter 4 Algorithms: In this chapter, we have discussed various machinelearning algorithms used for meta-modeling, multi-objective optimization and sensitivity analysis. In this work, we have used several commercial software as well as a few in-house developed codes that has been developed by members of our research group over the years. Here, we used HYBRID code developed by Professor G.S. Dulikravich and Professor M. J. Colaço, Surrogate modeling code developed by Dr. S. Choudhury and Evolutionary Neural Network (EvoNN) and BiObjective Genetic Programming (BioGP) code developed by Professor N. Chakraborti. Two of the most popular commercial optimization software in the world, IOSO (license provided by Professor I.N. Igorov) and modeFRONTIER (license provided by Professor C. Poloni) were used simultaneously. Statistical software, IBMSPSS, WEKA and R-Studio were used for statistical modeling, 
pattern recognition within the dataset. Apart from these, we used commercial metallurgical database, Factsage and information from ab-initio calculation based, Materials Project, for this work in screening the alloys. A brief introduction of these software, their advantages and limitations and how to effectively use them in our work has been reported in this chapter.

Chapter 5: Results 1 - Supervised learning: In this chapter, we have discussed our findings from meta-modeling followed by sensitivity analysis of models and its significance in this study. Thereafter, we have presented our findings from multi-objective optimization of targeted properties and its role in improvement of properties. Thereafter, we have discussed on MCDM approach and the way we have been using it in the present work.

Chapter 6 Results 2 - Unsupervised learning: In this chapter, we have discussed our findings from PCA and HCA analysis and its importance.

Chapter 7 Discussions: This chapter has been divided in two parts. In part 1 , we have compared the results from data-driven approaches. In part 2, we have reported salient features observed during characterization of magnets.

Chapter 8 Conclusions: In this chapter, we have summarized our findings from various approaches and focused on unique contributions. Additionally, we have listed a set of tasks that we could not address in our present work due to time and funding constraints but we think that it is important to discuss. 


\section{CHAPTER 2 LITERATURE REVIEW}

\subsection{Magnetic terminology}

Permanent magnets create a magnetic field around itself in free space, and usually do not need any continuous supply of energy for maintaining this field. Magnetism is a result of the motion of electrons around the nucleus. The resultant magnetic moment is a result of orbital motion and spin motion. Atoms that have incomplete shells possess a permanent magnetic moment. These moments interact with each other and align themselves parallel to each other. On application of external magnetic field, net magnetic moment in a magnet induces a force to align the magnetic moment with the applied magnetic field. This phenomenon is observed predominantly in two groups of elements: $3 d$ elements $(\mathrm{Cr}, \mathrm{Mn}, \mathrm{Fe}, \mathrm{Co}$, $\mathrm{Ni})$ and $4 \mathrm{f}$ elements (Ce, Nd, Sm, Eu, Gd, Th, Dy, Ho, Er, Tm). $4 f$ elements mentioned here belong to Lanthanides or are also known as Rare-earth elements (REE). $\mathrm{Ce}, \mathrm{Nd}$, and Sm are light rare earth while Gd and Dy are heavy. Of these, $\mathrm{Ce}$ is the most abundant (less critical) while the other rare - earth are critical in terms of supply. Rare-earth elements have superior magnetic properties due to the presence of unpaired $4 \mathrm{f}$ electrons (Cullity and Graham, 2009).

Any materials can be classified into three different groups based on its response to the applied magnetic field. Ferromagnetism is strongest of all. Ferromagnetic materials are strongly attracted by external magnetic field. At elevated temperature, also known as Curie temperature, thermal energy exceeds exchange interaction. Thus, the material loses its magnetic properties and becomes paramagnetic. Paramagnetic materials are also attracted towards the 
applied magnetic field, but the attraction is several times weaker than the ferromagnet. Diamagnets are least affected by external magnetic field and the attraction is several times weaker than the ferromagnets and paramagnets. Diamagnetism is exhibited by all classes of materials, but in case of ferromagnets and paramagnets, diamagnetic effect is negligible.

Ferromagnetic materials can be further classified into Hard and soft magnets. Hard magnetic materials have a tendency of retaining magnetic properties after removal of applied magnetic field, whereas soft magnetic materials lose their magnetic properties as soon as the applied magnetic field is removed. Magnetization can be defined as a measure of induced magnetic dipole moments. Its unit is Gauss or Tesla.

From application point of view, two properties are most important, namely Remanence $\left(B_{r}\right)$ and Coercivity $\left(H_{c}\right) . B_{r}$ is measured in gauss or Tesla and can be defined as the amount of magnetization retained by a magnet after removal of applied magnetic field. Coercivity is measured in Oersted and is a measure of resistance to applied magnetization. It can be defined as an amount of magnetic field required to demagnetize a magnet. Another important property that is basically dependent on both $B_{r}$ and $H_{c}$ is magnetic energy density. It is also referred as maximum energy product $\left((B H)_{\max }\right)$. It can be defined as an amount of magnetic energy stored in a magnet. Its unit is gauss-Oersted. A higher $(B H)_{\max }$ will require less materials and will be helpful in synthesizing small magnets with superior magnetic properties. 
$(B H)_{\max }$ is mathematically the area of the largest rectangle that can be inscribed in the second quadrant of the B-H curve (Kramer et al., 2012). $H_{c}$ and $B_{r}$ are conflicting, that is, one has to sacrifice on one of these properties to improve the other property. Therefore, in order to maximize $(B H)_{\max }$, one needs to optimize $H_{c}$ and $B_{r}$. Hence, we are left with a problem with three conflicting objectives that has to be maximized simultaneously.

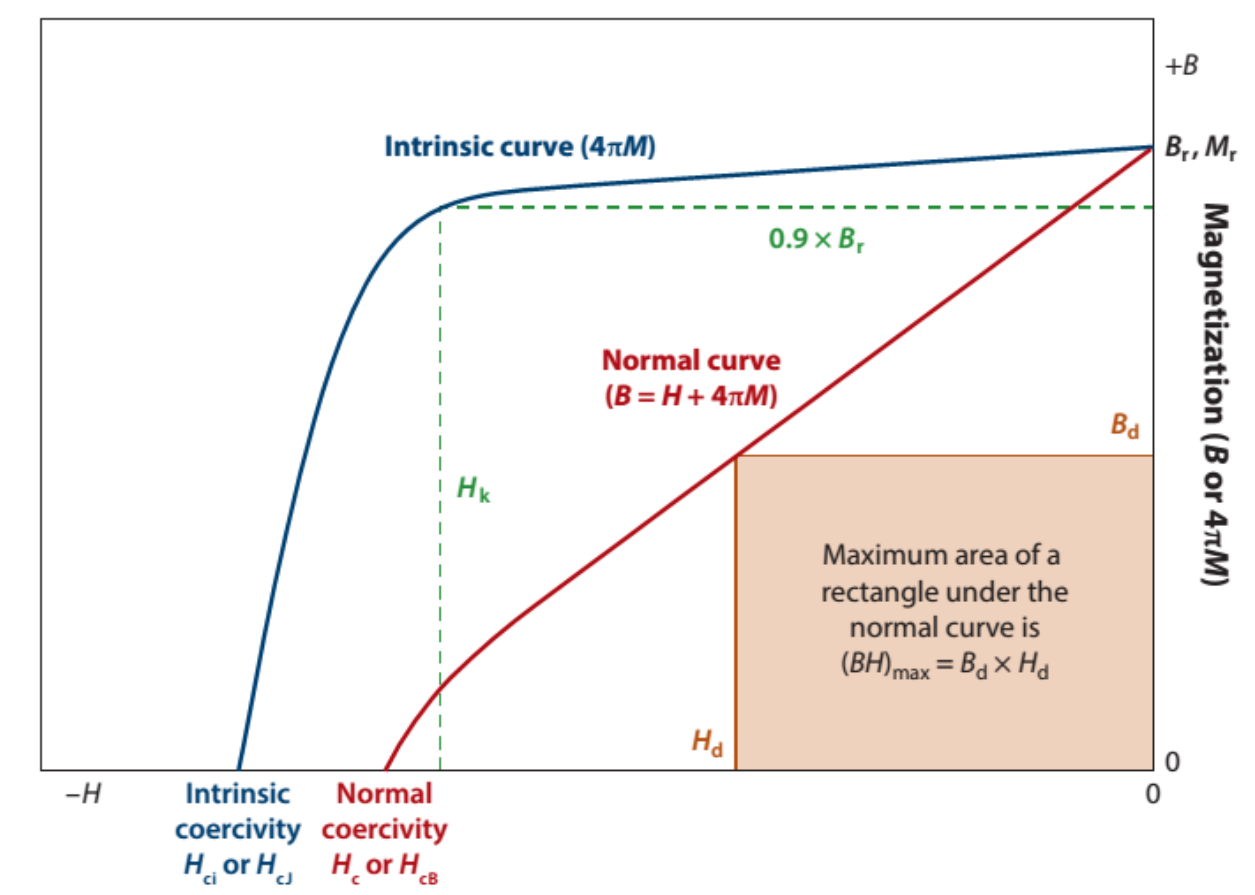

Field $(\boldsymbol{H})$

Figure 4: B-H curve: shows relation between $H_{c}, B_{r}$ and $(B H)_{\max }$. (Kramer et al., 2012)

\subsection{Magnetic AINiCo alloys}

The first step in the discovery of AlNiCo alloys was made by Mishima and his group in Japan (Cullity and Graham, 2009) in 1931. Initially, it belonged to the Fe-Ni-Al based system. In later years, researchers went for cobalt and copper 
additions. In those times it was predominantly Fe-Co-Ni-Al based quaternary system. This was popularly known as AINiCo 5 . In later years, titanium was added in various amounts (3-8) and showed remarkable improvement in $H_{c}$ but at the expense of $B_{r}$. This led to the development of AINiCo 8 magnets and still it has a highest $H_{c}$ among the AINiCo grades. Magnetic properties in these magnets were attributed to the presence of a two-phase system, $\alpha_{1}$ and $\alpha_{2}$, of Body Centered Cubic (BCC). It was later observed that separation of $\alpha_{1}$ and $\alpha_{2}$ phases is due to a metallurgical phenomenon popularly known as spinodal decomposition. Phase $\alpha_{1}$ is Fe-Co rich ferromagnetic phase and $\alpha_{2}$ is $\mathrm{Ni}$-Al rich phase. Phases $\alpha_{1}$ and $\alpha_{2}$ are stable up to $850{ }^{\circ} \mathrm{C}$ that is just below the Curie temperature, which is about $860^{\circ} \mathrm{C}$. Above $850^{\circ} \mathrm{C}$, Face Centered Cubic (FCC) $\gamma$ phase begins to appear and it was observed in a few samples (Dilon, 2014). The $\gamma$ phase must be avoided, as it is detrimental for magnetic properties. Various attempts (such as modification of heat treatment protocol and addition of various alloying elements) have been made to stabilize the magnetic $\alpha_{1}$ and $\alpha_{2}$ phases and simultaneously eliminate or reduce the amount of $\gamma$ phase. In the past few decades, (especially after the discovery of powerful REE-based magnets in 1980 's), there has been limited research on AINiCo magnets. The recent rise in prices of rare earth elements led to the search for rare-earth free magnets. In recent years, AINiCo magnets are again a popular choice for research mainly due to their proven high-temperature stability and related properties at an affordable 
cost (Dilon, 2014), (Xing et al., 2013). Currently, AINiCo alloys are not limited to quaternary systems and may contain eight or more elements (Cullity and Graham, 2009), (Jha et al., 2014). In this work, we selected eight elements, namely Iron (Fe), Cobalt (Co), Nickel (Ni), Aluminum (Al), Titanium (Ti), Hafnium (Hf), Copper $(\mathrm{Cu})$ and Niobium $(\mathrm{Nb})$. Variable bounds of these elements have been tabulated in Table 1. From both experimental as well as modeling point of view, it will be helpful to discuss the role of these alloying elements. This information can be utilized to select meta-model for targeted properties. This will be helpful in developing a knowledge base for discovery of new materials and/or improving properties of existing materials.

The following text will provide the reader with a brief idea regarding the role of various alloying elements and its effect on $\left(H_{c}\right)$ and $B_{r}$ (Dilon, 2014), (Jha et al 2016a)

- Cobalt: It is a $\gamma$ stabilizer. A solutionization anneal is needed to homogenize it to a single $\alpha$ phase. Cobalt increases $H_{c}$ and Curie temperature.

- Nickel: It is also a $\gamma$ stabilizer. Hence, solutionization anneal temperature needs to be increased in order to homogenize it to a single $\alpha$ phase. Nickel increases $H_{c}$ (less than Cobalt) while decreases $B_{r}$.

- Aluminum: It is an $\alpha$ stabilizer. It will be helpful in reducing the solutionization anneal temperature. Aluminum is expected to affect $H_{c}$ positively.

- Copper: It is an $\alpha$ stabilizer. Research shows that Copper affects Hc and $\mathrm{Br}$ positively and increases it. In AINiCo 8 and 9, Cu precipitates out of the $\alpha_{2}$ 
phases into particles and is responsible for the magnetic separation between $\alpha_{1}$ and $\alpha_{2}$ phases. An increase in these phase separations leads to an increase in $H_{c}$

- Titanium: It is an $\alpha$ stabilizer and one of the most reactive elements. It reacts with impurities such as $\mathrm{C}, \mathrm{S}$, and $\mathrm{N}$ and purifies the magnet by forming precipitates with these elements. It helps in grain refining and inhibits columnar grain growth. Majority of grains is aligned perpendicular to the chill plate due to columnar grain growth and large shape anisotropy can be achieved if spinodal decomposition occurs in this direction. Titanium increases $H_{c}$ at the expense of $B r$.

- Niobium: It is an $\alpha$ stabilizer. It forms precipitate with Carbon. Carbon is a strong $\gamma$ stabilizer and needs to be eliminated. Nb also inhibits in columnar grain growth. $\mathrm{Nb}$ increases $\mathrm{Hc}$, at the expense of $\mathrm{Br}$.

- Hafnium: It is used for retaining magnetic properties at high temperatures. It precipitates at the grain boundary and helps in improving creep properties. Recent studies related to Co-Hf magnets (Sellmyer et al., 2013), motivated us to use $\mathrm{Hf}$ in this study.

From the above literature, the reader can understand the role that spinodal refining plays in the improvement of the properties of these magnets. Several research groups have developed their theories for improved properties of these magnets. $(B H)_{\max }$ is dependent on both $B_{r}$ and $H_{c}$ and it is proportional to $H_{c}$ at low $H_{c}$. For instance, a recent study of nanostructured magnetic material suggests that it is possible to reach a very high magnetic energy product for fine wires of the 
decree of $10 \mathrm{~nm}$ (Dilon, 2014). Directionally aligned rods obtained because of shape anisotropy due to spinodal decomposition in AlNiCo alloys were approximated as such fine wires. As per this theory, the upper bound of $(B H)_{\max }$ was theoretically calculated and was found to be an order of magnitude greater than the best commercially available AINiCo alloy. According to this theory, $(B H)_{\max }$ is directly proportional to $M_{r}$ (Remanence magnetization), while $M_{r}$ is directly proportional to $M_{s}$ (saturation magnetization). Thus, the lower bound of $(B H)_{\max }$ is proportional to $\mathrm{Hc}$, and the upper bound of $(B H)_{\max }$ has been reported to be proportional to $M_{s}$. It must be noted that $H_{c}$ is an extrinsic property, while $M_{s}$ is an intrinsic property of the magnet. Thus, experimentalists have to be extremely careful while preparing specimens and designing thermomagnetic treatment protocols. They also must have access to advanced diagnostic tools required for analysis at nanometer scale. Two recent papers (Zhou et al., 2014), (Xing et al., 2014) reported the importance of copper rich precipitates between adjacent $\alpha_{1}$ phases and their importance in improvement of magnetic properties for AINiCo 8 and 9 grade alloys. 


\section{CHAPTER 3 RESEARCH PROBLEM AND METHODOLOGY}

In this chapter, we have discussed our research problem, our approach to solve the problem and the reason to rely on a certain approaches for improvements. We used a set of computational tools to develop a novel approach for the design and optimization of high-temperature, high-intensity permanent magnetic alloys without REE's.

The steps involved in the proposed approach can be listed as follows:

1. Initial 80 alloys: Our first task was to generate the dataset to manufacture the first batch of 80 alloys. We referred to the open literature for guideline for choosing the elements and then defined the variable bounds for these elements from our own expertise. Sobol's algorithm (Sobol, 1967), one of the bestknown quasi-random number generators were used to explore the variable space for new alloy composition that has not been reported in the literature. Alloying elements and variable bounds has been tabulated in Table 1 and Table 2. These alloy compositions were then screened on the basis of limited knowledge of phase equilibrium and magnetic properties from a commercial thermodynamic database, Factsage (Bale et al., 2002), (Factsage, 2015). 
Table 1: Concentration bounds AINiCo type alloys

\begin{tabular}{|l|c|c|c|}
\hline & \multicolumn{3}{|c|}{ Variable bounds (weight percent) } \\
\hline Alloying elements & $\mathbf{1 - 8 5}$ & $\mathbf{8 6 - 1 4 3}$ & $\mathbf{1 4 4 - 1 8 0}$ \\
\hline Cobalt (Co) & $24-40$ & $24-38$ & $22.8-39.9$ \\
\hline Nickel (Ni) & $13-15$ & $13-15$ & $12.35-15.75$ \\
\hline Aluminum (Al) & $7-9$ & $7-12$ & $6.65-12.6$ \\
\hline Titanium (Ti) & $0.1-8$ & $4-11$ & $3.8-11.55$ \\
\hline Hafnium (Hf) & $0.1-8$ & $0.1-3$ & $0.095-3.15$ \\
\hline Copper (Cu) & $0-6$ & $0-3$ & $0-4.5$ \\
\hline Niobium (Nb) & $0-2$ & $0-1$ & $0-1.5$ \\
\hline Iron (Fe) & \multicolumn{3}{|c|}{ Balance to 100 \% } \\
\hline
\end{tabular}

Table 2: Design cycles and alloy numbers

\begin{tabular}{|c|c|c|}
\hline Cycle number & Number of Alloys Designed & Best alloy (number) \\
\hline 1 & $1-80$ & 30 \\
\hline 2 & $81-85$ & 84 \\
\hline 3 & $86-90$ & 86 \\
\hline 4 & $91-110$ & 95 \\
\hline 5 & $111-120$ & 117 \\
\hline 6 & $120-138$ & 124 \\
\hline 7 & $139-143$ & 139 \\
\hline 8 & $144-150$ & 150 \\
\hline 9 & $151-160$ & 157 \\
\hline 10 & $161-165$ & 162 \\
\hline 11 & $166-173$ & 169 \\
\hline 12 & $174-180$ & 180 \\
\hline
\end{tabular}


2. Manufacture and testing: These alloys were synthesized by our research collaborators at North Carolina State University. A brief account on manufacturing protocols, testing methods and characterization tools used in this work has been reported in section 3.1. The alloys were tested for various properties of interest as reported in Table 3. This dataset will be used for developing meta-models for targeted properties.

Table 3: Quantities to be simultaneously extremized using multi-objective optimization

\begin{tabular}{|l|c|c|}
\hline Properties & Units & Objective \\
\hline Magnetic energy density $\left((B H)_{\max }\right)$ & $K g \cdot m^{-1} s^{-2}$ & Maximize \\
\hline Magnetic coercivity $\left(H_{c}\right)$ & Oersted & Maximize \\
\hline Magnetic remanence $\left(B_{r}\right)$ & Tesla & Maximize \\
\hline Saturation magnetization $\left(M_{s}\right)$ & Emu/g & Maximize \\
\hline Remanence magnetization $\left(M_{r}\right)$ & $\mathrm{Emu} / \mathrm{g}$ & Maximize \\
\hline$\left((B H)_{\max }\right) /$ mass & $\mathrm{m}^{-1} \mathrm{~s}^{-2}$ & Maximize \\
\hline Magnetic permeability $(\mu)$ & $K g \cdot m \cdot \mathrm{A}^{-2} \mathrm{~s}^{-2}$ & Maximize \\
\hline Cost of raw material & $\$ / \mathrm{Kg}$ & Minimize \\
\hline Intrinsic coercive field $\left(j H_{c}\right)$ & $A \cdot m^{-1}$ & Maximize \\
\hline Density( $\rho)$ & $K g \cdot m^{-3}$ & Minimize \\
\hline
\end{tabular}


3. Meta-modeling (Response surface generation): Meta-models were developed to link alloy composition to desired properties mentioned in Table 3. A commercial optimization package, modeFRONTIER (ESTECO, 2015) was used for this purpose.

(a) Scaling of dataset: This is an important step in meta-modeling so that the meta - model gives equal importance to all the variables/alloying elements. In our problem, one can see that variable bounds differ for all the elements. Hence, in this part we scaled the data set from 0-1.

(b) Training and Testing set: Meta-modeling or surrogate modeling can be classified as a supervised machine learning algorithm approach. Hence, one needs a training set to train the model to discover various correlations between the variables and targeted properties and a testing set to test the model for data that it has not been exposed to. In this work, we divided the initial dataset randomly with 75 percent (60 alloys) in the training set and 25 percent ( 20 alloys) in the testing set.

(c) Selection of meta-model: Selection of response surface methodology approaches to develop meta models are one of the trickiest part. Due to limited information on this subject in the current multi-component system, overdependence on any one approach can mislead us. Looking at the complexity of the problem, it was decided to use a set of response surface methodologies to develop meta-models. These approaches include Radial basis functions (RBF), Gaussian Processes (GP), Kriging, Anisotropic Kriging (AKR), and Evolutionary Design. 
(d) Sensitivity analysis: Meta-models were tested for their capability in determining composition-property relationship. We found that our meta-models were able to mimic the limited knowledge we have from the literature for a few elements and associated properties. These models were given a preference while selection of metamodels for the next step. As the dataset is quite noisy, we were left with quite a few mixed responses which is quite usual for multi-component systems. Hence, the models needed to be further tested on various accuracy measures like R-Squared, RAAE, RMAE and the most accurate one was chosen for further study. Various approaches were used to develop response surfaces. Meta-models selected were used for multi-objective optimization and also for predicting other properties of new candidate alloys.

4. Multi-objective optimization: Ten bulk properties that need to be optimized for implementation are listed in Table 3. Due to software limitations, we could efficiently optimize three properties at a time. In the present case, these three properties were $B_{r}, H_{c}$ and $(B H)_{\max }$. From the literature review in Chapter 2, it is known that these three properties are conflicting, hence multi-objective optimization will prove to be an asset in this case. Several optimization runs were performed to get a diverse pool of results. Various optimization algorithms were used for this purpose in order to efficiently search the variable space for optimized properties. It includes Non-dominated Sorting Genetic Algorithm II (NSGA2), MultiObjective Particle Swarm Optimization (MOPSO), Multi-Objective Simulated Annealing (MOSA) and fast optimizer which uses response surface to speed up the optimization process using various search algorithms mentioned above. 
5. This work was independently carried out by our collaborators at three different places using:

(a) Commercial optimization package, Indirect Optimization based on Self-Organization (IOSO) algorithm (Egorov-Yegorov and Dulikravich, 2005).

(b) Hybrid response surface (Dulikravich and Colaço, 2015) was used because of its robustness, accuracy and computational efficiency. Multiobjective optimization was performed by Non-Dominated Sorting Genetic Algorithm (NSGA2) (Deb, 2001).

(c) Surrogate model selection algorithm (Dulikravich and Colaço, 2015) was used because of its robustness and simplicity.

Pareto-optimized predictions from the above optimization packages were merged. From the available chemical composition of Pareto-optimized alloys, we predicted the 7 properties listed in Table 3 that were not optimized. Now, we have a new set of alloys and the next task was to screen them so as to manufacture a few specimens for testing.

6. Multi-Criterion decision making (MCDM): In MCDM, all the 10 properties can be simultaneously optimized at the same time. We already have a set of alloys whose properties were predicted by most accurate meta-models, of which three of these properties were optimized several times. In MCDM, the expert can use his understanding of the problem and then specify his needs and run the optimization so as to find a set of alloys with properties that can be accepted for implementation. In the present case, we have been using MCDM to screen our predicted results and selected a few alloys for manufacture and testing. 
7. This work has been performed in cycles to check upon improvements. Steps 2-5 were repeated in each of the cycles until the improvements of multiple macroscopic properties of such magnetic alloys became negligible.

8. Unsupervised learning: Unsupervised learning methods like Principal component analysis (PCA) and Hierarchical cluster analysis (HCA) were used to analyze the dataset for pattern recognition that will help us to determine composition-property relations that can be supported from experiments/literature. This was done in order to find influential alloying elements for development of a knowledge base. At the same time, the sensitivity analysis also helps in finding the least influential alloying elements that could be discarded to make way for introduction of affordable and readily available rare-earth elements.

9. Thermodynamic approach: Candidate alloys were also screened for phase stability over a range of temperature from a thermodynamic database, FACTSAGE. This will be helpful to the experimentalist in designing thermomagnetic protocol for improved results. Thereafter, we analyzed possibility of rareearth additions through ab-initio based calculations from another open source database, Materials Project (Materials Project, 2014).

10. Experimental modeling and characterization: Two samples were manufactured and thermo-magnetic protocol was modified for improved results. Thereafter, through advanced characterization techniques, we were able to quantify the effect of Titanium additions on the evolution of $\mathrm{Cu}-\mathrm{Ni}$ rich bridges between adjacent $\alpha$ phases that is needed for improved magnetic properties. 
In this work, we have addressed several issues that an experimentalist faces during the design of new alloys. Here, we have used a set of computational tools to address various issues and also reported the reason for selecting a certain approach. This work will be helpful in developing a knowledge base that will be useful to the research community in designing new alloys. In data-driven material science, knowledge discovery (Rajan, 2013) for designing new materials requires:

1. Data: In this work, our database is a combination of randomly generated experimentally verified data and Pareto-optimized predictions.

2. Correlations: Various linear and nonlinear correlations were discovered by using a set of supervised and unsupervised learning algorithms to discover various trends in the dataset.

3. Theory: Our findings have been backed up from the literature for quite a few properties. This information can be coupled with theoretical knowledge to motivate the experimentalist to modifying standard manufacturer protocol for the design of new alloys. Advanced characterization further helped us in determining various correlations that have been reported on our work.

\subsection{Experiments}

As mentioned before in this chapter, the alloys were manufactured at North Carolina State University. This work is their propriety and has been submitted in the form of a technical report to AFOSR. In this work, we have added a brief account regarding the standard protocol followed by the group. The reader is advised to refer the following paper for a better understanding (Fan et al., 2016b; 2016a). 
Steps involved in the manufacture of these alloys can be listed as follows:

1. Manufacture: Bulk samples were cast in a water cooled copper hearth. The specimens were re-melted at least three times to ensure homogenization.

2. Thermo-magnetic treatment: As cast samples were solutionized at $1250 \mathrm{C}$, and then thermos-magnetically treated at $800^{\circ} \mathrm{C}$ for 10 minutes. Magnetic field (3T) was applied in the direction of cylindrical axis.

3. Hysteresis measurements: were performed by Quantum Design superconducting quantum interference device (SQUID) magnetometer, where magnetic field varied between $-3 \mathrm{~T}$ to $+3 \mathrm{~T}$ at room temperature. $\mathrm{Br}, \mathrm{Hc}$ and $(B H)_{\max }$ were obtained from hysteresis loops obtained in this step.

4. Structural and compositional properties were analyzed by:

(a) Transmission electron microscope (TEM).

(b) Energy-dispersive x-ray spectroscopy (EDS) analysis. 


\section{CHAPTER 4 ALGORITHMS}

Famous statistician GEP Box quoted, "Essentially all models are wrong, but some are useful"(Box and Draper, 1987). Even though some are useful, over dependence on one can be misleading. Hence, a person working with metamodels must have an idea regarding the pros and cons of the model he is using for his work. In this chapter, we will discuss the need for developing models in materials science along with ways to develop them

\subsection{Data-driven materials science}

Most of the Engineering design problems are real world problems and depend heavily on experimental and / or simulation to evaluate various design objective and constraint function and accordingly predict the behavior when the variables are altered. On one hand, collecting sufficient experimental data is quite time consuming and may cost a fortune, on the other hand simulations are computationally expensive and in some cases even a single simulation may take several minutes, hours or even days. These above limitations will prohibit even routine tasks like design optimization, sensitivity analysis etc. as it may require thousands or even millions of simulations to come at a meaningful conclusion. Hence, the need of the hour is to construct an approximate model that will somehow emulate the behavior (or try to capture the basic trends) of the system/simulation model while being computationally affordable to evaluate.

In the present context, development of new alloys or even improving the properties of existing alloys is a challenging task mainly due to limited experimental database. In order to address this problem, research over the last few years is 
focused primarily on developing accurate data-driven models. Due to its potential and robustness, these models have been successfully implemented by researchers in the past and forms the core of recently established materials genome initiative funded by US government. In order to work with data, one needs to focus on three basic steps:

1. Generate new data,

2. Provide necessary guidelines to manage the existing data and

3. Utilize the existing data efficiently.

From the implementation point of view, one such approach is by developing data-driven models. Data-driven models use actual production data and by means of various concepts and makes an attempt to mimic the behavior/functionality of the system. At the same time, successful implementation requires accurate predictions. Since, the experimental data of a physical system are quite noisy, data-driven models are associated with a certain degree of uncertainty. Researchers over the years have proposed a number of ways to address this problem and because of it, there has been significant improvement in accuracy of these models. Over the years, there has been a growing trend of using several concepts of artificial intelligence in order to address this problem. Machine learning algorithms are one such branch of artificial intelligence that has been successfully implemented in materials science by several research groups around the world, including our group (Egorov-Yegorov and Dulikravich, 2005), (Datta et al., 2013), (Mueller et al., 2015), (Jha et al., 2014), (Jha et al., 2014a), (Jha et al., 2015). A few successful implementations include prediction of phase diagrams, 
material properties determine composition-property relationship, the development of inter-atomic potentials to make a few.

Machine learning algorithms that have been successfully employed in the materials science domain can be categorized as Supervised Learning and Unsupervised Learning algorithms. Supervised learning is usually associated with multi-objective optimization (Mueller et al., 2015). A few basic terms associated with data-driven models can be listed as follows:

1. Training data: It is the initial set of data, which is needed for datadriven modeling. The data can be from original experiments or from simulations. In supervised learning, this training data consists of input and output. Efforts are being formed to produce models that will be able to link these input to the production. While in Unsupervised learning, there is no output. Here, the purpose is to find patterns among the input.

2. Testing dataset: This can be used to test the accuracy of the model. In supervised learning, while training, the model was not exposed to this set of data. Hence, this dataset can be used to test the accuracy of the model. If the expert is satisfied with a certain level of accuracy, then they can use these models to test even new inputs that the expert thinks can yield better results. In unsupervised learning, this data can be utilized in a different way. One can check for new patterns within the dataset. Thereafter, see that if matches with the previous observations. This testing dataset can then be merged with the previous data set. In supervised learning, one can use this merged dataset to develop new 
models, while in unsupervised learning, one can use this dataset to discover new patterns and observe the shift in behavior of the system.

A few examples of both of these methods and its successful implementation can be listed as follows: Supervised learning algorithms like Artificial Neural Networks (ANN), Support Vector Machines (SVM), Genetic Programming (GP)have been successfully used in the past. These algorithms were used to predict processing-structure-property relationship, predict and classify crystal structures, develop model Hamiltonian (Mueller et al., 2015).

Unsupervised learning algorithms like Principal Component Analysis (PCA), Hierarchical Cluster Analysis (HCA); K-means clustering have been used to address a few important features regarding the dataset. These algorithms can be used to analyze composition spreads, analyze micrographs and noise reduction in the data sets.

In order to optimize the performance of a data-driven model, the use of evolutionary algorithms can be helpful. Hence, in this chapter, we have discussed various supervised learning algorithms followed by various evolutionary algorithms used by us and other algorithms that have the potential to improve the current results. At the end, we have discussed a few unsupervised learning algorithms.

\subsection{Supervised learning}

In this part, we have discussed various methods of developing data-driven models. These models have also been referred as Surrogate models or Response Surface Models (RSM) or meta-models. Surrogate models are basically datadriven models that require an initial set of experimental data to construct the model. 
Hence, RSM can be defined as a statistical measure that takes into account the quantitative data from experiments to determine the behavior of the system and can be utilized to solve multi-variant equations. The RSM / surrogate models can be utilized for various applications such as

1. To determine the factors (or system variables) that will satisfy a set of desired specifications in order to understand the behavior of the system under consideration.

2. Design optimization: To determine various combinations of factors (or variables) that will yield a desired response surface and estimate the response near the optimum.

3. Sensitivity analysis: To determine the effect of variation of factors (or variables) on any specific response over the region of interest.

4. Uncertainty Analysis: To determine and analyze any specific response over the region of interest for various combinations of factors (or variables) which were not tested while development of the model.

\subsubsection{Surrogate Models/ Meta-Models}

In this section, we have discussed Radial Basis Functions (RBF), Kriging (KG), Gaussian Processes, Genetic Programming and Artificial Neural Networks (ANN) along with their practical application and limitations.

\section{Radial Basis Functions (RBF)}

It is a real valued function, whose value depends on the distance from the origin or any other center and any function satisfying this property can be termed as radial function. The distance is usually Euclidean distance, while some other 
metric can also be used. RBFs are one of the most popular mesh-free kernel approximation techniques. Initially, RBFs were developed for scattered multivariate data and function interpolation. Later, it was found that RBFs were able to construct an interpolation scheme with favorable properties such as high efficiency, good quality and capable of dealing with scattered data, especially for higher dimensional problems. It is well known that a good interpolation scheme also has great potential for solving partial differential equations, and RBFs have been used for this purpose (Dulikravich and Colaço, 2015).

The general form of an RBF can be written as shown in equation 4.1.

$$
y(x)=\sum_{i=1}^{N} \omega_{i} \phi\left(\left|x_{i}-x_{j}\right|\right)
$$

$\phi\left(\left|x_{i}-x_{j}\right|\right)$ is the radial function based on the Euclidean norm between the $i^{\text {th }}$ and $j^{\text {th }}$ point and $\omega_{i}$ are the appropriate weights found by solving the system of equations. Broadly, RBF can be classified into two main groups:

1. The globally supported ones, namely the Multiquadrics $(\mathrm{MQ}$, $\sqrt{\left(x_{i}-x_{j}\right)^{2}+C_{j}^{2}}$ where $C_{j}$ is a shape parameter), the inverse multiquadrics, thin plate splines, Gaussians, etc.

2. The compactly supported ones such as the Wendland family.

Some commonly used RBF formulations used in this work has been summarized below in Table 4. 
Table 4: RBF approximation used in this work

\begin{tabular}{|l|l|}
\hline Type of approximation & $\phi\left(\left|x_{i}-x_{j}\right|\right)$ \\
\hline Multiquadrics (MQ) & $\sqrt{\left(x_{i}-x_{j}\right)^{2}+C_{j}^{2}}$ \\
\hline Inverse Multiquadrics(IMQ) & $\left|\sqrt{\left(x_{i}-x_{j}\right)^{2}+C_{j}^{2}}\right|^{-1}$ \\
\hline Gaussian & $\exp \left[-C_{j}^{2}\left(x_{i}-x_{j}\right)^{2}\right]$ \\
\hline
\end{tabular}

\section{Advantages of using RBF}

1. Wide range of application and has superior performance for highorder non-linear problems tested for large-scale/ small-scale data.

2. It has been successfully applied both for continuous and discrete response functions.

3. It can be approximated as a single layer type of ANN usually referred as radial basis function network and has been used as a kernel for Support Vector Machines.

On the other hand, in the absence of a polynomial term that is orthogonal to the RBF, its performance is relatively poor outside the fitting set.

\subsubsection{Kriging Model (KG)}

KG models are widely applied to approximate irregular data and it was initially developed for geostatistical applications. The approximation function is a combination of Global and local approximation model. That is a combination of a polynomial function and its departure. It can be represented as equation 4.2. 


$$
\begin{gathered}
\hat{y}=\sum_{i=1}^{N} \beta_{i} f_{j}(x)+Z(x) \\
\operatorname{Cov}\left[Z\left(x_{i}\right), Z\left(x_{j}\right)\right]=\sigma^{2} R\left(x_{i}, x_{j}\right)
\end{gathered}
$$

Where, is a n-dimensional vector, $f(x)$ is an approximation function defined on a global level. $Z(x)$ is the local departure from the global model and is assumed to be a realization of a stochastic process with mean zero and spatial correlation function given by equation 4.3. The Gaussian correlation function is the most popular and is widely used while other correlation functions can likewise be applied. Usually, $f_{j}(x)$ is a constant term. The behavior of the Kriging model is heavily controlled by a covariance function called a variogram. The modeFRONTIER software package allows for use of Gaussian, Exponential, Matern and Rational Quadratic variograms (modeFRONTIER, 2015).

In the present study, the training points were interpolated using a Gaussian random function as the covariance function to estimate the trend of these stochastic processes. This correlation between $Z\left(x_{i}\right)$ and $Z\left(x_{j}\right)$ is heavily dependent on the distance between points $x_{i}$ and $x_{j}$. In this case, a special weighted distance is used rather than Euclidean distance as in the case of RBFs.

\section{Advantages of Kriging}

1. Since the KG function consists of both a trend function and its deviation, it is quite useful for predicting spatially correlated data.

2. It is quite flexible due to availability of a wide range of correlation functions. 
3. It provides a basis of a stepwise algorithm to determine critical system variables and the same data can be utilized for building the predictive model.

\section{Limitations of Kriging}

1. Construction of the model is time consuming and it adds to the computational cost.

2. For non-linear and high dimensional problems, computational time is high if the initial data set is large.

3. There is a possibility that correlation matrix may become singular if the sample points are placed close to one another or are generated from a particular design.

\subsubsection{Gaussian Processes}

In recent years, Gaussian processes have emerged as a potential competitor to ANNs for developing regression models. Over the years, Bayesian approaches have been successfully implemented for developing regression models based on ANNs, as well as Gaussian prediction models. Gaussian processes are based on Bayesian probability distribution approach. In other words, it can be considered as generalized Gaussian probability distribution model. Nevertheless, these procedures are best fitted for non-polynomial responses. In the following text, we present a general description of the algorithm for a process where the mean is assumed to be zero. For a given dataset corresponding to a non-linear function $y(x)$, input vectors $X_{N}$ and output vectors $t_{N}$ are denoted in equation 4.4 


$$
X_{N}=\left[x_{1}, x_{2}, x_{3}, x_{N}\right] ; t_{N}=\left[t_{1}, t_{2}, t_{3}, t_{N}\right]
$$

Posterior probability distribution of $y(x)$ can be expressed as denoted in equation 4.5 .

$$
P\left(y(x) \square t_{N}, X_{N}\right)=\frac{P\left(t_{N} \square y(x), X_{N}\right) * P(y(X))}{P\left(t_{N} \square X_{N}\right)}
$$

In order to predict the future values of $t$, it is important to know the assumed prior distribution of the function, $P(y(X))$ and the assumed noise model, whereas the parametrization of the function $y(X \square W)$ is irrelevant (for a parameter $W$ ). The basic idea is to place the prior over the space of the function without parameterizing. In this case, the simplest type of prior will be termed as Gaussian process. Gaussian processes are specified by mean and covariance functions in the same way as Gaussian distribution has the mean and covariance matrix. Here, the mean is a function of $x$, and the covariance can be estimated by evaluating the function $y(x)$ at point $x$ and $x^{\prime}$. Thus, the expected covariance can be denoted as $C\left(x, x^{\prime}\right)$.

\subsubsection{Shepard-K-Nearest}

It is one of the most popular algorithm for partitioning and clustering. In $\mathrm{K}$ Nearest algorithm, interpolation is based on the $\mathrm{K}$ nearest designs to the candidate points. Its behavior is similar to that of a plain Shepard method or the Mollifier Shepard method except that it only takes into account user specified number of nearest neighbor points, $K$, into calculations. It works on the basis of using the weighted sum of the $K$ nearest points as expressed in equation 4.6 


$$
\begin{aligned}
& S(x)=\sum_{i=1}^{N} \omega_{i}(x) f\left(x_{i}\right) \\
& \omega_{i}(x)=\frac{\mathrm{P} x-x_{i} \mathrm{P}}{\sum_{i=1}^{K} \mathrm{P} x-x_{i} \mathrm{P}^{-p}}
\end{aligned}
$$

It can be seen from equation 4.7, that the weights are obtained by the normalized inverse power $p$ of the distances. In this study, $K$ was kept constant as 11 and $p$ as two for all of the test cases (modeFRONTIER, 2015).

\subsubsection{Artificial Neural Networks (Basheer and Hajmeer, 2000)}

Artificial Neural Network (ANN) is an attempt by researchers to mimic the functionality of the complex nervous system of a human brain, with ANN being its simplest representation. A simple representation of ANN consists of an input layer, a hidden layer, and an output. The hidden layer processes the information provided by the input node $(x)$ and weights associated $(w)$ with the connection between the input node and the node in the hidden layer. This information is transferred to the output via a transfer function. The transfer function for final output $y(x)$ is usually a hyperbolic tangent function. Each node in the hidden layer is associated with a bias value $\left(w_{0}\right)$.

Equations 4.8 and 4.9 show the output of a single neuron $g$ and $y(x)$. The processing in the hidden layer has been often unexplained and it seems to be that the information is processed inside a black box. This brings about non-linearity in the output due to which the results obtained are new and non-intuitive. Due to this property, an ANN can outperform statistical methods like linear regression. Hence, 
an ANN is able to fit highly non-linear functions that cannot be fitted by other conventional techniques.

$$
\begin{gathered}
g=\sum_{j=1}^{n} w_{j} x_{j}+w_{0}=W^{T} X \\
y(x)=\operatorname{sigmoid}(g)=\frac{1}{1+\exp ^{w^{T} X}}
\end{gathered}
$$

The ANN used in this study is based on a classical feed-forward ANN with a single hidden layer. The ANN networks are trained by Levenberg-Marquardt algorithm. The reader can refer to the user manual for detailed understanding of the training process followed in the ANN model of modeFRONTIER. In the present case, the research team used the default setting of the optimizer, that is, the number of nodes in the hidden layer was set by the optimizer and not the user (modeFRONTIER, 2015).

4.2.6 Evolutionary Design (Poli, Langdon and McPhee, 2008), (modeFRONTIER, 2015)

Genetic Programming is an extension of evolutionary algorithm 4.2.11, that allows computer to automatically solve the problems. It was introduced by John Koza. It has emerged as a potential competitor for ANN for developing data driven models. Here, randomly generated Computer Programs represent potential solutions. It exempts human from designing complex algorithms for creating programs that give desired optimal solutions. The model is trained as symbolic trees, which are evolved by evolutionary algorithms. Well-defined structure specific crossover and mutation operators exist for the tree representation. 
Two important things that have to be looked upon while evolving a tree (computer program) are:

1. Depth of the tree and

2. Training error associated with it

As we increase the depth (complexity), there is improvement in the performance of the tree on the training error part. However, beyond a certain complexity, it may be possible that there is no further improvement in its performance and executing such tree will add to the computational cost. Another major problem associated with such a tree is that it will over fit the data. On the other hand, if the complexity is beyond a certain level, training error will increase and it may under fit the data and thus the basic trends may not be captured.

Evolutionary design is a version of genetic programming that is used in modeFRONTIER to evolve functions on the basis of user defined parameters like depth of tree, crossover probability, population, number of generations and the function nodes to be used, etc. We get a set of expressions (solutions) for a particular objective function out of which the one with the lowest error is chosen (modeFRONTIER, 2015).

\subsubsection{HYBRID}

The HYBRID method in this study combines the fittest polynomial RBF and the Kriging formulation into one hybrid method. Here, fittest polynomial RBF is used as the transfer function in the DACE (Design and Analysis of Computer Experiments) Kriging formulation. This form of Kriging formulates the correlation function as shown in equation 4.10 (Dulikravich and Colaço, 2015). 


$$
\phi\left(\left|x_{i}-x_{j}\right|\right)=\sum_{i=1}^{n} \Theta_{k}\left|x_{i}-x_{j}\right|^{P_{k}}
$$

Here, both $\Theta_{k}$ and $P_{k}$ have to be optimized.

\subsubsection{Performance measurements of a metamodel}

The performance of each meta-modeling technique can be measured on the following aspects (Dulikravich and Colaço, 2015):

1. Accuracy: capability of predicting the system response over the region of interest.

2. Robustness: capable of achieving good accuracy for different problem types and sample sizes.

3. Efficiency: computational effort required for constructing the metamodel and for predicting the response from a set of new points of meta-models.

4. Transparency: capability of illustrating explicit relationships between input variables and responses.

5. Conceptual simplicity or ease of implementation. Simple methods should require minimum user input and be easily adapted to each problem.

To provide a more complete picture of meta-model accuracy, three different metrics were used, namely R Square, Relative Average Absolute Error (RAAE), and Relative Maximum Absolute Error (RMAE): 
1. R Square $\left(R^{2}\right)$

$$
R^{2}=1-\frac{\sum_{i=1}^{N}\left(y_{i}-\hat{y}_{i}\right)^{2}}{\sum_{i=1}^{N}\left(y_{i}-y_{i}^{\prime}\right)^{2}}=1-\frac{M S E}{\text { Variance }}
$$

Where, $\hat{y}_{i}$, is the corresponding predicted value for the observed value $y_{i}$ and $y_{i}^{\prime}$ is the mean of the observed values. While MSE (Mean Square Error) represents the departure from the meta-model of an ideal simulation model, the variance captures how irregular the problem is. $R^{2}$ must be high and it has been widely associated with meta-model prediction accuracy.

2. Relative Average Absolute Error (RAAE)

$$
R A A E=\frac{\sum_{i=1}^{N} \mid\left(y_{i}-\hat{y}_{i} \mid\right.}{n^{*} S T D}
$$

Where STD stands for standard deviation. The smaller the value of RAAE, the more accurate the meta-model.

3. Relative Maximum Absolute Error (RMAE)

$$
R M A E=\frac{\max \left(\mid\left(y_{1}-\hat{y}_{1}|,|\left(y_{2}-\hat{y}_{2}|,|\left(y_{3}-\hat{y}_{3}|, \ldots,|\left(y_{n}-\hat{y}_{n} \mid\right)\right.\right.\right.\right.}{\hat{a}}
$$

Large RMAE indicates a large error in one region of the design space, even though the overall accuracy indicated by $R^{2}$ and RAAE can be very good. Therefore, a small RMAE is preferred. However, since this metric cannot show the overall performance in the design space, it is not as important as $R^{2}$ and RAAE. 
Single Variable Response (Pettersson et al, 2007), (Giri et al, 2013)

Single Variable Response (SVR) has been a methodology often used for qualitative analysis of the training results obtained from Evolutionary Neural Network (EvoNN) and Bi-Objective genetic programming (BioGP) (Pettersson et al., 2007), (Jha et al., 2015b). In SVR, a style of variation is created by generating values between zero and one on time scale. The trend line is irregular, that is there are regions of constant values, sharp increases and sharp decreases in the line. This has been referred to as input signal in the following text. Here, an input signal is furnished for each variable (alloying element). The response of that signal (that corresponds to that particular variable) was checked with respect to the input signal for the objectives and constraints trained through the selected model. For SVR testing, the input signal (trend of variation) was used for one of the variables, while the other variables were kept constant at an average value. The model output response was plotted against the variable trend. The various responses were tabulated for each of the models.

Following terminologies were used in SVR testing:

1. Direct: This means that the model output increases on increasing the value of the input signal and decreases on decreasing the value.

2. Inverse: This means that a particular variable will affect the model output in the opposite manner. That is, if we quantitatively increase/decrease the value of that particular variable (concentration of this alloying element), it will result in decrement/increment in the value of the corresponding property of the alloy. 
3. Nil: This means that the model was unable to find any correlation between that particular variable and the model output.

4. Mixed: This means that the model has a different response to a different set of data of any particular variable.

A model may show Direct response to a particular variable in a certain region (data set), while the same model may show Inverse response in the other region or even Nil response in another region. Since the experimental data set is noisy, this behavior is expected. Corresponding tables and figures have been included for additional information in this regard in the model development part.

\subsubsection{Multi-objective optimization}

Most of the practical, real world problems involve more than one objective, which are more or less of conflicting in nature and needs to be satisfied in order to solve any particular problem. An ideal multi-objective optimization problem deals with a number of objective functions. Generally, we have more than one objective that is to be optimized, i.e. maximized or minimized simultaneously. These problems may include design constraints that need to be satisfied by all the members of the solution space. In mathematical terms, a multi-objective problem can be expressed as shown in equation 4.15 to 4.17 (Deb, 2001).

$$
\begin{aligned}
& \text { Maximize, Minimize, } F_{m}(X), m=1,2, \ldots . M \\
& g_{i}(X) \geq 0, j=1,2, \ldots J \\
& h_{k}(X)=0, k=1,2, \ldots K \\
& x_{i}^{L} \leq x_{i} \leq x_{i}^{U}, i=1,2, \ldots . .
\end{aligned}
$$


The solution $X$ is a vector of $n$ decision vectors defined as $X=(x 1, x 2, x 3, \hat{a}$. While solving a multi-objective optimization problem one has to deal with design constraints, i.e. physical limitations, time bounds, etc. that must be satisfied by every member of the solution space. In the above case, these constraints are introduced as variable bounds on $x_{i}, J^{\text {th }}$ inequality and $K^{\text {th }}$ equality constraints. Each variable $x_{i}$ has to be within a lower bound $x_{i}^{L}$ and an upper bound $x_{i}^{U}$ as mentioned in the problem. Similarly, $g_{i}(X)$ and $h_{k}(X)$ are the inequality and equality constraints respectively. All solutions that lie in this constrained variable space (also known as a feasible region of the Search space) are known as feasible solutions. All solutions that do not satisfy the prescribed $(J+K)$ constraints and $2 n$ variable bounds are known as infeasible solutions.

\section{Concept of Dominance and Pareto-Optimality}

Multi-objective optimization algorithms use the concept of dominance. In these algorithms, any two solutions are compared based on their relative function values on whether one solution dominates the other or not (Deb, 2001).

A solution $x^{(1)}$ can dominate another solution $x^{(2)}$, if

1. The solution $x^{(1)}$ is no worse tha, $x^{(2)}$ in all objectives, i.e. $f_{j}\left(x^{(1)} \geq f_{j}\left(x^{(2)}\right.\right.$ for all $j=1,2 \hat{a}$

2. The solution $x^{(1)}$ is strictly better than in at least one objective , i.e. $f_{j}\left(x^{(1)}>f_{j}\left(x^{(2)}\right.\right.$ for at least one $j \varepsilon 1,2 \hat{a}$ 


\section{Pareto-Optimal Set}

In a given set of feasible solutions $P$, the solutions are compared among them for non-dominance. The non-dominated set of those solutions $P^{\prime}$ are solutions that cannot be dominated by any member of the set $\mathrm{P}$. If $P$ corresponds to the entire search space then the set, $P^{\prime}$ can be referred as the Pareto-Optimal set.

Pareto solutions are those for which improvement on one objective can only occur with the worsening of at least one other objective i.e. the objectives are conflicting. Thus, instead of a unique solution to the problem, the solution of a multi-objective problem is a set of solutions referred at as Pareto set, or Pareto front.

\section{Local Pareto-Optimal Set}

During optimization, there exists several sets of solutions that are nondominated with respect to each other in that particular set yet they do not dominate the entire search space. These sets of non-dominated solutions in the search space are referred as Local Pareto-Optimal Set.

\section{Global Pareto-Optimal Set}

It is the non-dominated set of points of an entire feasible search space. It is also referred as Pareto-Optimal set and it consists of the best possible solutions of the entire search space which are non-dominated with respect to all the solutions in the entire search space. 


\subsubsection{Evolutionary Algorithms for Multi-Objective Optimization}

Classical optimization methods usually convert the multi-objective optimization problem to a single-objective optimization problem thus emphasizing on one particular set of Pareto-optimal solution at a time. When such a method is used for finding multiple solutions, it has to be applied many times. In addition, there is a possibility of finding solutions in the vicinity of the pre-existing solution (that we obtained in the previous simulation) in each simulation run thus affecting diversity. Thus, it is difficult to ensure that the solutions that we have can be considered as the global Pareto-optimal set even after multiple runs.

These drawbacks of classical optimization methods can be taken care of in Evolutionary algorithms by:

1. The population approach helps in finding multiple solutions.

2. Niche preserving methods help in maintaining diversity.

Unlike conventional optimization techniques, evolutionary algorithms use population based approach thus making them capable of evolving multiple solutions simultaneously that approaches the non-dominated Pareto front in a few runs. The genetic operator operating on this population, i.e. Recombination (crossover) and Mutation alters the structure of the solutions in such a way that there is a good chance that the newly evolved solutions may lie in the previously unexplored part of the search space. This helps in maintaining diversity among the solutions and helps in checking that the evolved solution set may not prematurely converge to a Local Pareto-optimal set of solutions. These abilities of EA make them suitable to find a diverse set of solutions for difficult problems with 
discontinuous and multi-modal solution spaces. In addition, most multi-objective EA does not require the user to have prior knowledge of the physical parameters and governing equations that affect the problem that they are dealing with. Their features make EA, one of the most popular heuristic approaches to deal with multiobjective design and optimization problems.

\subsubsection{Evolutionary Algorithms}

The Evolutionary Algorithms (EA) or Genetic Algorithm (GA) are heuristic search algorithm which are basically inspired by the Darwinian theory of evolution based on the survival of the fittest. In the process of natural evolution, superior individuals are evolved by the process of natural evolution along with improvement in their performance in subsequent generation at the same it does not discard an inferior population members. Similarly, in GA, while evolving a solution, superior candidates are generally given preference in the selection process in order to enhance their performance in subsequent generation while inferior members are not completely discarded and a part of it is retained in order to maintain diversity (Deb, 2001).

Genetic algorithms belong to the larger class of evolutionary algorithms (EA), which generates solutions for optimization problems using techniques inspired by natural evolution, such as selection, crossover and mutation,. A population of individuals (possible solutions) is bred through a certain number of

generations (iterations) depending upon their fitness values while the genetic 
operators like selection, crossover and mutation operates on the whole population. At the end of the GA run, the individuals left are the best possible solution for the problem that evolved in subsequent generations.

\section{Basic terminology}

Key components of EA include:

1. Population: The first step while using GA is to initialize a Population. All the individuals in the predefined feasible search space constitute the population. An individual member of a population represents the possible solution of the optimization problem. The architecture of all the individuals in a particular population must be same that is, it can be either binary or real-coded. Depending on the architecture the other genetic operators, that is, selection, crossover and mutation are defined.

2. Fitness: In order to differentiate between the individuals of a population, each individual of the population is assigned a scalar value, fitness, which denotes its importance in the population.

3. Selection: Selection operator selects individuals in the population on the basis of their fitness for reproduction. The higher the fitness of the individual, the higher is its probability to get selected for reproduction. In some cases (elitist $\mathrm{GA}$ ), the best individuals are just copied into the next generation without altering its structure. This is done in order to preserve some of the best evolved individuals (elite) otherwise the structure of these individuals (elite) may get altered and these individuals (Elite) may be completely lost when GA operators operate on them in subsequent generation. 
4. Crossover or Recombination: At any time, two individuals (parent) are selected for reproduction to create two offspring (children). The evolved individuals after crossover will have a different structure and thus different fitness value. Usually, crossover probability is kept high $(\leq 0.8)$.

5. Mutation: Mutation is usually performed after crossover. Here, an individual is selected randomly and its structure is altered by a small amount. Mutation can prove to be beneficial in some cases where a small change in the structure is needed to achieve the desired solution. Different types of mutation operators are in practice for both binary and real coded individuals. Mutation probability is kept low $(\leq 0.3)$.

6. Elitism: It refers to the methodology applied to saving the best evolved individuals (elite) in any particular generation so that it is not affected by the genetic operators that operates on the whole population in that generation. These individuals (elite) may have taken generations to evolve and it may be possible that some small alteration that has been introduced in their structure by the genetic operators operating on them may destroy the complete information that they contain. This may make the algorithm some sort of random search and we may fail in getting the desired set of Pareto-optimal solutions. In order to avoid this situation, the term, elitism is introduced. One of the possible ways of introducing elitism is by mixing the parents and the offspring after every generation and then sorting out the best individuals to replace the previous parent solution. One can also make copies of the best individuals of a particular generation and retain it for 
the next generation and then apply genetic operators on the remaining population thus saving them from getting destroyed.

7. Generation: in mathematical terms, it refers to, iteration. In GA, usually a number of generations has been used as stopping criterion if any other stopping criterion is not defined.

\subsubsection{Non-Dominated Sorting Genetic Algorithm (NSGA-II)}

It is an elitist Non-Dominated Sorting Genetic Algorithm is popularly known as NSGA-II. In NSGA-II, an elite preserving strategy along with a diversity preservation mechanism ensures better spread of the solution (Deb, 2001).

The various steps involved in the algorithm are as follows:

1. The first step involves defining population, which may consist of randomly generated possible solutions within the feasible search space.

2. This population is sorted into different non-domination levels and each of the solution is assigned a fitness that is equal to its non-domination level.

3. Crowding distance is calculated for each of the members of the population.

4. Binary tournament selection is performed in order to select individuals for recombination (crossover) where the criterion for selection is the calculated crowding distance.

5. Recombination and mutation operators are used to evolve an offspring population of the same size that is equivalent to the size of the initial parent population. 
6. Both the parent and the offspring population are mixed together and non-dominated sorting is performed on the combined population. Based on dominance criterion the population is sorted in different non-dominated fronts where each of the solution is assigned to a font that is equal to its non-domination level.

7. The parent population is now replaced by the non-dominated fronts in an increasing order starting from the front 1 . The last front which cannot be fully occupied is arranged according to the crowding distance of the solutions comprising it in a decreasing order of magnitude. The left over positions are filled from this sorted list from the top. The leftover solutions in this front along with the other fronts that cannot be included in the population are discarded.

8. This loop is repeated by going to step 2 and continuing till the termination criterion is reached.

A few unique features that were introduced in this algorithm are discussed in the following text.

\section{Elitist Preserving Strategy}

In any particular generation, parents and the offspring are mixed after crossover and mutation operations. This combined population is subjected to nondominated sorting. The parents of the previous generation are replaced by these new fronts as discussed above. So the best evolved solutions (elite) of the previous generation have a good chance to find a place on one of the fronts. Hence, the elites of the previous generation get a chance to be a part of the next generation thus preserving them. 


\section{Crowding Distance Selection}

It gives an idea about the density that is the number of points surrounding a particular point on the Pareto front in the objective space. To estimate the density (crowding), the average distance between the two points lying on either side of the concerned point along each of the objectives is taken into consideration. These two points on either side of the concerned point (for which crowding distance is to be measured) lie on the opposite corners of the cuboid of largest size that can be constructed in the objective space without including any other point apart from these three. The distance between these two opposite points is taken as the crowding distance of the point in between. A larger crowding distance helps in preserving diversity and, thus, a better spread of solutions is obtained.

Another way involves a methodology in which none of the fronts are assured full representation in the new population. In this way extra spaces are created and thus it gives a chance to solutions on the front of lower rank to be part of the new population, thus helping in preserving diversity and assuring a better spread of the solution.

\subsubsection{Evolutionary Strategies}

Evolution strategies (ES) were developed by Rechenberg and later modified by Schwefel (Rechenberg, 1971)), Schwefel, 1974), (modeFRONTIER, 2015). It is also referred to as the German version of GA due to its origin. Earlier versions were usually restricted to Two-membered ES due to the complexity involved in the evolution of the solution which made the process time consuming. 
It differs from conventional GA in basically two ways:

1. It uses real parameter values and

2. It does not use a crossover like operator.

In the absence of crossover, it uses selection and mutation quite efficiently in order to evolve a solution. This version of the mutation is known as Self adaptive mutation where the extent of mutation differs from generation to generation depending upon the perturbation needed to get an optimum solution. Later, multimembered ES (MMES) was introduced comprising of multiple members. Another variant, Recombinative ES explores the benefits of crossover.

\section{Multi-Membered ES (MMES)}

It can be classified in two ways as shown in equation 4.18 and 4.19 .

$$
\begin{gathered}
(\mu+\lambda)-E S \\
(\mu, \lambda)-E S \\
y^{j}=x^{i}+N(O, \sigma)
\end{gathered}
$$

Here, $\mu$ is the size of the initial population, $\lambda$ is the number of offspring to be generated from $\mu$ members of the initial population.

The mutated solution $y^{j}$ is created from the initial population member according to equation 4.20. Here, $O$ is the mean, and $\sigma$ is the Standard deviation or Strength of Mutation. $N(O, \sigma)$ denotes Normal distribution of $O$ and $\sigma$ 
In equation 4.18 , after the evolution of $\lambda$, both parent and offspring population are added and out of these, best $\mu$ members are chosen for the next generation.

While in equation 4.19 , the best $\mu$ members for the next generation are chosen from the evolved set of $\lambda$ offspring only and the rest is discarded.

\subsubsection{Particle Swarm Optimization}

Particle Swarm Optimization (PSO), was proposed by Kennedy and Eberhart and it belongs to the broader class of swarm intelligence techniques that are used to solve optimization problems (Kennedy and Eberhart, 1995), (modeFRONTIER, 2015). The main aspect in which it differs from other evolutionary algorithms is the fact that here there is no selection operator. This means, all members of the population are given equal importance and there is no specific preference for any member on any basis. It is a population based stochastic technique basically inspired by the natural behaviors observed in flocks of birds or schools of fish. In PSO, simple potential solutions referred as particles, moves in the search space of an optimization problem under consideration. In the initialization phase, each of the particles is assigned with a random initial position and an initial velocity. This algorithm also keeps track on the particle that is leading the entire flock at any point of time. Each of the particles memorizes the position of the best solution that they found and position of the global leaders. Each particle uses its own experience and the experience of its neighbor particles to choose the manner in which it must move in the search space. At the end of each iteration, each particle updates its velocity on the basis of its own best performance so far 
and the global best performance of the swarm as a whole. This velocity is a weighted sum of three components: the old velocity, a velocity component that drives the particle towards the location in the search space where it previously found its best solution so far and a velocity component that drives the particle towards the location in the search space where the neighbor particles found the best solution so far that is the global best performance of the swarm as a whole.

The velocity $\left(V_{i}^{t+\Delta t}\right.$ and position $\left(x_{i}{ }^{t+\Delta t}\right.$ of the $i^{\text {th }}$ particle at time $t$ are updated to time $t+\Delta t$ according to the following two equations respectively:

$$
\begin{aligned}
V_{i}^{t+\Delta t} & =\omega V_{i}^{t}+R_{1} \tau_{1}\left(V_{i, I B S T}{ }^{t}-V_{i}^{t}\right)+R_{2} \tau_{2}\left(V_{i, G B S T}{ }^{t}-V_{i}^{t}\right) \\
x_{i}^{t+\Delta t} & =x_{i}^{t}+V_{i}^{t+\Delta t}
\end{aligned}
$$

Where, $\omega$ denotes user defined inertia weights, $\tau$ terms are the used defined constants, while $R$ terms are random numbers generated uniformly in the range $[0,1]$. The term $V_{i, I B S T}{ }^{t}$ denotes the individual best performance of the particle so far while $V_{i, G B S T}{ }^{t}$ denotes the global best performance while considering all the particles of the swarm. In equation 4.22 , the first term is responsible for the inertia effect while the second and the third term are responsible for the acceleration effects.

\section{Multi-Objective Particle Swarm Optimization}

Multi-Objective Particle Swarm Optimization (MOPSO): PSO is one of most successful artificial/engineering swarm intelligence system and has been applied to many problems of different domains. There exist a few variations of it 
for solving multi-objective optimization problem. In this work, we used the version from optimization software, modeFRONTIER (modeFRONTIER, 2015).

\subsubsection{Simulated annealing}

The term annealing basically refers to the process of slowly cooling of molten substance (Kirkpatrick et al, 1983). If we heat a solid to its melting point and then cool it, the structural properties of the solid depend on the rate of cooling. If the liquid is cooled quickly (quenched), then crystals will contain imperfections. However, if the melt is cooled slowly enough, large crystals will be formed thus making it feasible for the atoms to attain minimum energy configuration. At any equilibrium temperature $\mathrm{T}$, the atomic energies $(\mathrm{E})$ of a substance are distributed according to the Boltzmann equation, where $\mathrm{k}$ is the Boltzmann constant.

Simulated annealing is basically a search algorithm and not an evolutionary algorithm. It is inspired by the Metropolis algorithm (Metropolis et al, 1953). In Metropolis algorithm, Boltzmann equation is used as a selection probability for acceptance of uphill moves in a search space. Here, downhill moves are also accepted whereas uphill moves are accepted only if a uniformly distributed random number in the interval $[0,1]$ is less than the value of the exponential term shown in equation 4.24 . In equation $4.24, d$ is basically the energy difference that is the difference between the uphill objective function value and the function value of the base point. The value of $\beta$ is problem dependent and it has to be determined empirically, while $T$ is the temperature. It can be seen that $\Theta$ decreases as $d$ increases or $T$ decreases. Uphill moves are given a small preference in order to search the complete search space and get the exact activation energy curve. 


$$
\begin{gathered}
P(E)=\exp \left(\frac{E}{k T}\right) \\
\Theta=\exp \frac{-d \cdot \beta}{T}
\end{gathered}
$$

\section{Multi-Objective Simulated Annealing (MOSA)}

SA was originally developed to use only one searching agent and in a few cases it worked better than EA for single objective optimization. It was hardly used for multi-objective optimization due to its inability to find multiple points, which is a major violation of the basic concept of multi-objective optimization that is to find a well distributed set of solutions known as Pareto front. Multi-Objective SA (MOSA) uses the concept of domination and the annealing scheme for efficient search and to find multiple solutions SA repeating the trials as it converges to the global optima with a uniform probability distribution in the single objective optimization. When there are two global optima, it can be proved that SA can find each optimum with

probability of 0.5 . MOSA can find a small group of Pareto solutions in a small interval of time and thereafter repeat the trials for finding additional solutions required in order to get the final Pareto front (modeFRONTIER).

\subsection{Unsupervised learning}

As the name suggests, there are no basic guidelines for these algorithms, hence it is unsupervised. These algorithms can be used to discover various pattern, divide the data into various clusters, reducing the dimensionality of the dataset for viewing, which may help researchers in better understanding of the physics of the problem. Here, an expert needs to be careful while choosing a 
certain algorithm and associated parameters for a specific case. Additionally, an expert needs to be very careful while interpreting the findings from these algorithms. One must use the technical aspects regarding the basic physics of the problem so that their results are meaningful and for it to be accepted by the materials research specialists for implementation. In this part, we have introduced two algorithms that we found suitable for our data set.

\subsubsection{Clustering Analysis and related algorithms}

Clustering analysis is usually done to find various patterns that may exist in the dataset. A cluster consists of a set of data points, which are similar to the other data points within the same cluster while dissimilar to data points in the other clusters. In most cases, similarity criterion is the Euclidian distance between the data points.

\section{Hierarchical Cluster Analysis (HCA)}

In HCA (Mueller et al., 2015), clustering begins with each data point within a cluster. These clusters are iteratively merged to form larger ones and finally merged as one large cluster. In this work, clustering was done by the Ward's approach while there are several other alternatives for the same (ESTECO, 2015), (IBMSPSS, 2015). The final result is a tree-like structure called Dendrogram, which shows the way the clusters are related. User can specify a distance or number of clusters to view the dataset in disjoint groups. In this way, the user can get rid of a cluster that does not serve any purpose as per his expertise. In this case, we used 
MVA (Multivariate data analysis) node in optimization package: modeFRONTIER (ESTECO, 2015) and other statistical software IBM SPSS (IBMSPSS, 2015) for HCA analysis.

Clusters are classified by following measures (ESTECO, 2015)

1. Internal similarity (ISim): It reflects the compactness of the k-th cluster. It must be higher.

2. External similarity (ESim): It reflects the uniqueness of the $k$ - th cluster. It must be lower.

3. Descriptive variables: are the most significant variables that help in identifying cluster elements that are similar to one another.

4. Discriminating variables: are the most significant variables that help in identifying cluster elements that are dissimilar to other clusters.

HCA analysis can be used to cross check the findings of SVR analysis mentioned above in the text.

\subsubsection{Principal Component Analysis (PCA)}

PCA can be classified as an unsupervised learning machine-learning algorithm [Mueller et al., 2015]. It was performed in order to determine correlations between variables and various properties by reducing the dimensionality of the dataset without losing much information. PCA uses an orthogonal transformation to convert a set of usually correlated variables (or properties) into a set of values of linear uncorrelated variables known as Principal Components (PCs). Hence, each PC is a linear combination of all the original descriptors (variables and properties). The first principal component (PC1) accounts for maximum variance 
in the dataset, followed by PC2 and so on (Rajan, 2013), (ESTECO, 2015). Thus, it is possible to visualize a high dimensional dataset by choosing first two or three principal components (Mueller et al., 2015). It is also used for identifying patterns in data, as patterns may be hard to find in high-dimensional data sets.

Prior to PCA analysis, three important terms need to be discussed for better understanding of the analysis results:

1. Scree plot: It is a plot between eigen values and component number. It is an important parameter used to select the number of components required to represent the complete dataset. Usually, components with eigen values above one (1) are chosen for further analysis. It can be seen from the figures in the later part that the scree plot usually flattens below eigenvalue 1 . This means that the later components do not have any significant effect on the dataset. Since, each successive component accounts for comparatively less variance, the least influential components can be ignored from further analysis.

2. Eigenvalues: are the variances of the principal components. Principal components analysis was conducted on the correlation matrix. Here, the variables were standardized, so that each variable has a variance of one, and the total variance is equal to the number of variables used in the analysis. Therefore, there will be eight PC for elements and nine PC for properties. The first component will always account for the most variance (and hence will have the highest eigenvalue). Next components will account for as much of the left over variance as it can. Hence, each successive component will account for comparatively less variance (hence less Eigen value) than the one leading it. 
3. Component plot: After the requisite numbers of components is chosen, these factors are plotted against each other, while the original variables (or properties) are diagrammed on this abbreviated space. The orientation of a certain variable (or property) on the reduced space determines its contribution towards a certain PC. That is, if the variable is positioned along PC1 on the 0 -line perpendicular to $\mathrm{PC}$, this variable will have maximum influence on PC1 and minimum influence on PC2. This will be better explained with the corresponding figures in the latter part of the text.

\subsection{Commercial software}

In this work, we used several commercial and open-source software. A brief description of this software has been provided below. Readers can refer to the references for better understanding.

\subsubsection{ESTECO: modeFRONTIER}

Esteco is the name of the software company that developed modeFRONTIER which is a multidisciplinary and multi-objective optimization tool. It can be paired with any Computer Aided Engineering (CAE) tool for creating a design of experimentation in accordance to our demand.

In this work, we used modeFRONTIER to develop meta-models, multiobjective optimization of targeted properties, MCDM, PCA and HCA analysis (modeFRONTIER, 2015).

\subsubsection{Indirect Optimization on the basis of Self-Organization (IOSO)}

IOSO is a semi-stochastic, multi-objective optimization algorithm incorporating certain aspects of a selective search on a continuously updated 
multi-dimensional response surface. The primary benefits of this algorithm are its outstanding reliability in avoiding local minima, its computational speed, and a significantly reduced number of required experimentally evaluated candidates alloys as compared to more traditional semi-stochastic optimizers such as genetic algorithms. Furthermore, the self-organizing response surface formulation used in IOSO allows for incorporation of realistic non-smooth variations of experimentally obtained data and provides for accurate insertion of such information. One of the advantages of this approach is the possibility of ensuring good approximating capabilities using minimum amount of available information. (Jha et al., 2014), (Egorov and Dulikravich, 2005).

In this work, we used it for meta-modelling and optimization.

\subsubsection{IBM SPSS}

IBM SPSS is a product of International Business Machines Corporation (IBM), where SPSS stands for Statistical Package for the Social Sciences. It is a commercial software package, applied for statistical analysis (IBM SPSS, 2015), (IBM SPSS, 2015a). SPSS is a widely used by market researchers, health researchers, survey companies, government, training researchers, marketing arrangements, data miners. In this work, we used it for PCA and HCA analysis.

\subsubsection{R Studio}

The studio is a detached and open-source Integrated Development Environment (IDE) for $\mathrm{R}$, a programming language for statistical computing and art. $R$ is a programming language and software environment for statistical 
computing and graphics supported by the R Foundation for Statistical Computing ( $R, 2016)$. In this work, we used R for PCA and HCA analysis.

\subsubsection{WEKA}

Waikato Environment for Knowledge Analysis (WEKA) is a popular suite of machine learning software written in Java, developed at the University of Waikato, New Zealand (WEKA, 2016). It is free software licensed under the GNU General Public License. Weka supports several standard data mining tasks, more specifically, data preprocessing, clustering, classification, regression, visualization, and feature selection. In this work, we used WEKA for clustering analysis.

\subsubsection{FACTSAGE}

FactSage, one of the largest fully integrated database computing systems in chemical thermodynamics in the world (FACTSAGE, 2015). In this work, we used FACTSAGE for performing phase equilibrium calculations of the magnets in a prescribed temperature range. It was used for screening of alloys prior to manufacture. 


\section{CHAPTER 5 RESULTS 1- SUPERVISED LEARNING}

In this chapter, we have discussed upon the results obtained from metamodelling and multi-objective optimization. We have worked through 12 cycles of design and optimization followed by experimental validation. Table 2 lists the alloys manufactured in each of the cycles and the best alloy in each cycle ranked on the basis of $(B H)_{\max }$ values. Work done in all the cycles is described as follows (Jha et al., 2016):

\subsection{Results over the design cycles}

1. Cycle 1 (Alloy 1-80): As already mentioned in section 3, initial compositions were predicted by Sobol's algorithm [Sobol, 1967]. A set of 80 elements was chosen for manufacture and testing. Measured properties were not according to our expectations.

We used this data set for development of response surfaces for the properties mentioned in section 3 by various methods described in section4. Thereafter, most accurate response surfaces were chosen. We proceeded further for multi-objective optimization of targeted properties (namely $(B H)_{\max }, H_{c}$ and $B_{r}$ ) in the hope of improved results to generate the next set of alloys. This work was simultaneously performed by our collaborators as mentioned in Chapter 3 . Pareto-optimized predictions were thereafter screened and we selected a set of 5 alloys for manufacture and testing.

2. Cycle 2 (Alloy 81-85): After experimental test, it was observed that, One of the predicted alloys (alloy \# 84) outperformed the initial set of alloys and 
the other predicted alloys. While the other four of these alloys performed similar to the initial 80 alloys. Figure 5, 6 and 7 shows the plots of the optimized properties over the cycles. It can be seen that the magnetic properties of alloy \# 84 is significantly better that the alloys present in the dataset which was used to develop meta-models. This demonstrates the efficacy of the current approach in using computational tools in materials design.

Hence, we moved forward and repeated the process (that is meta-modelling followed by multi-objective optimization and experimentation) in the hope of further improvements. Variable bounds were modified and the new bounds are listed in Table 1.

3. Cycle 3 (Alloy 86-90): In this cycle, alloy \# 86 was the best candidate and in the vicinity of alloy \#84, while the other four alloys in this cycle possessed magnetic properties similar to an initial set of alloys.

Variables (alloying elements) were plotted against each other to examine the distribution of alloying elements as can be seen in Figure 8, 9, 11 and 10. It can be observed that for alloy \# 1-90, alloys were not uniformly distributed in the variable space. Hence, the meta-model lacked support points in a certain region and it affected its overall accuracy. Additionally, there was no significant improvement over the previous cycle as alloy\#84 and 86 were similar in magnetic properties. Hence, we decided to generate the next set of alloys by Sobol's algorithm in order to improve distribution of elements in the variable space. This provided the response surfaces with more support points needed to develop accurate meta-models. 
4. Cycle 4 (Alloy 91-110): There was significant improvement in this cycle and alloy\# 95 was the best performer. Additional support points proved to be helpful in improving of response surface predictions. Alloy \#95 has an $H_{c}$ of 980 OeOeas compared to 750 Oe for the previous best alloy \#84). This improvement motivated us to proceed towards the next cycle of design and optimization task to generate alloy composition for the next cycle.

5. Cycle 5 (Alloy 111-120): We observed significant improvement in the properties of the new alloys, especially alloy 117 is the best alloy in terms of $(B H)_{\max }$. Alloy \# 111 and 114 has a $H_{c}$ of 1050 Oe and alloy \#117 reported 1000 Oe (as compared to 980 Oe for the previous best alloy \# 95). Thus we proceeded towards design and optimization task to generate alloy composition for the next cycle.

6. Cycle 6 (Alloy 121-138): We observed significant improvement in both $(B H)_{\max }$ and $H_{c}$. Alloy \# 124 was the best performer on both of these properties. Hence, we proceeded forward towards the next cycle of design and optimization task to generate alloy composition for the next cycle.

7. Cycle 7 (Alloy 139-143): In this cycle, Alloy \# 139 was the best performer. Its properties were in the vicinity of alloy 124 . There was no significant improvement in the desired properties. Design and optimization task was halted to minimize waste of resources. For the next set of alloys, we used our HYBRID response surface (Dulikravich and Colaço, 2015). 
Cycle 8-11 (Alloy 144-180): In these cycles, variable bounds were relaxed by 5 percent, while the methodology remains the same.

8. Cycle 8 (Alloy 144-150): Alloys composition was again generated in MAIDROC lab. There was marginal improvement in $H_{c}$, while we did not observe any significant improvement in other properties.

9. Cycle 9 (Alloy 151-160): This work was performed by our

collaborator, Dr. Souma Choudhury uses his in-house developed Surrogate model selection algorithm (SM). We did not observe any significant improvement in this cycle for any of the properties discussed in Table 3.

10. Cycle 10 (Alloy 161-165): Alloys composition was again generated in MAIDROC lab using modeFRONTIER. There was marginal improvement in $H_{c}$ , but no improvements in any other properties.

11. Cycle 11 (Alloy 166-173): Hybrid response surface and modeFRONTIER were used. There was marginal improvement in $H_{c}$, while we did not observe any improvement in other properties.

12. Cycle 12 (Alloy 174-180): Hybrid response surface and modeFRONTIER: There was marginal improvement in $H_{c}$, while we did not observe any improvement in other properties.

\subsubsection{Optimized properties}

Figure 5, 6 and 7 shows the comparison between various approaches for a set of properties that were optimized simultaneously namely $(B H)_{\max }, H_{c}$ and $B_{r}$. From these figures, we can see that our approach was able to recover from 
initial drawbacks and there was significant improvement in properties in subsequent cycles.

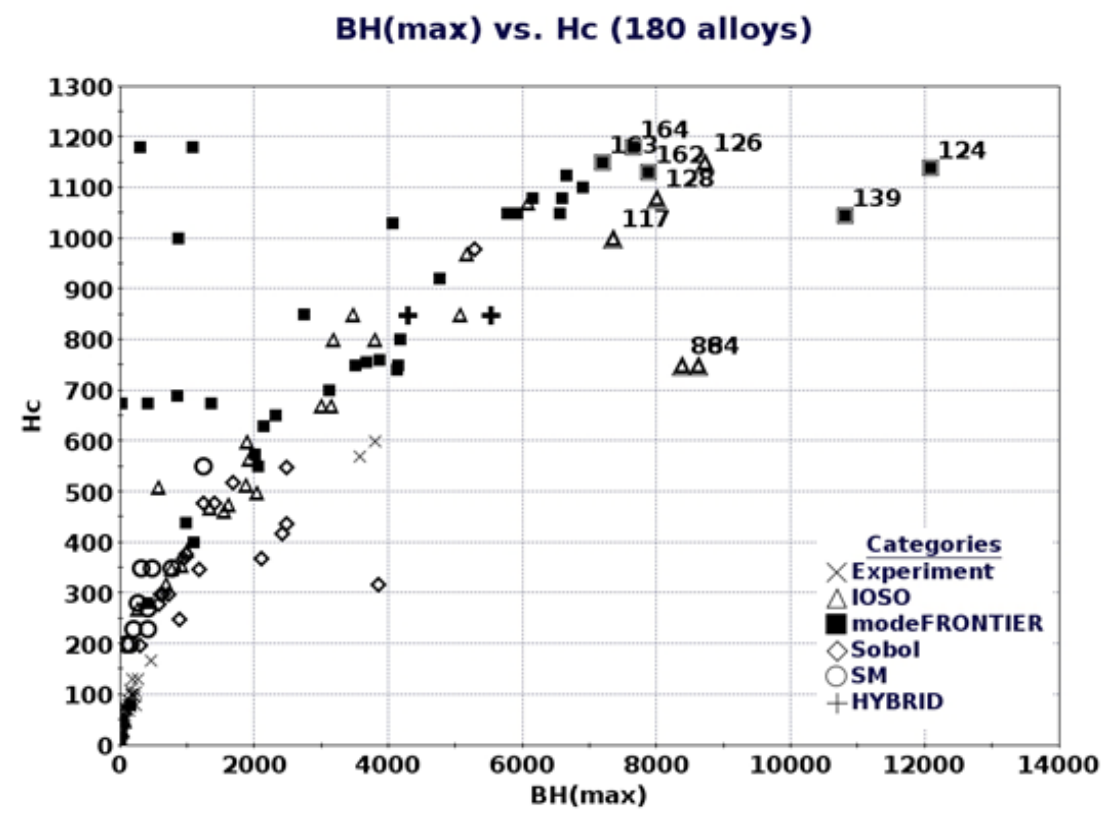

Figure 5: Magnetic energy density vs magnetic coercivity

$\mathrm{BH}(\max )$ vs. $\mathrm{Br}$ (180 alloys)

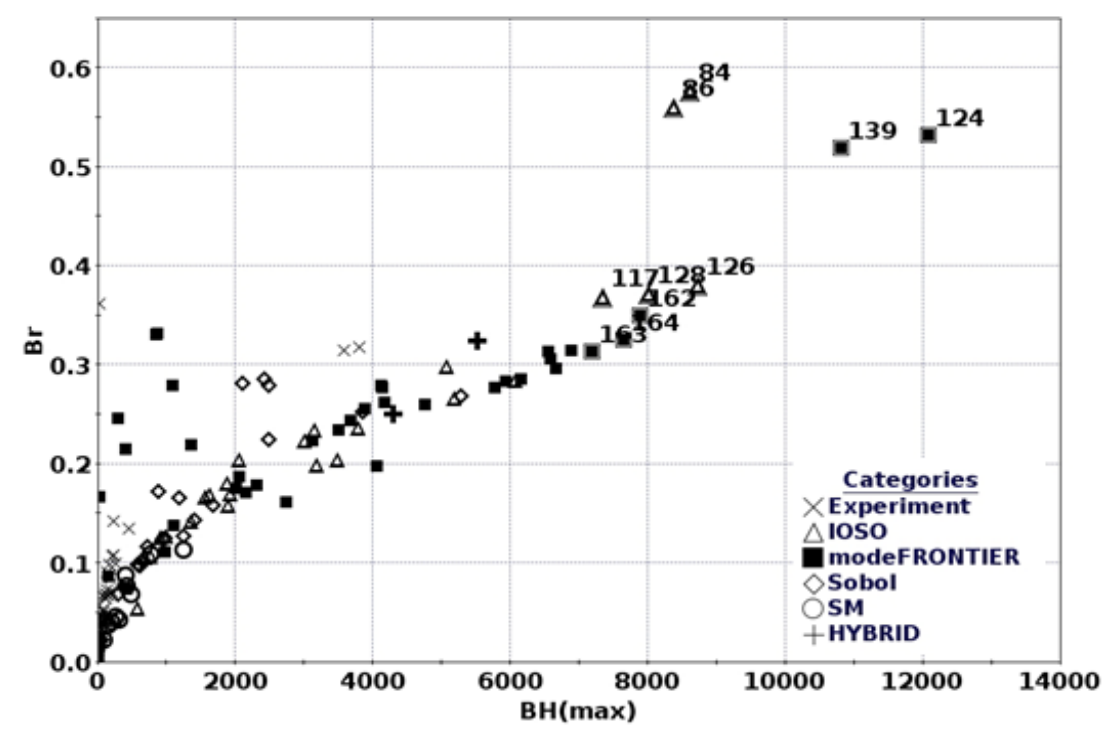

Figure 6: Magnetic energy density vs magnetic remanence, comparison of solutions by various approaches 
Hc vs. Br (180 alloys)

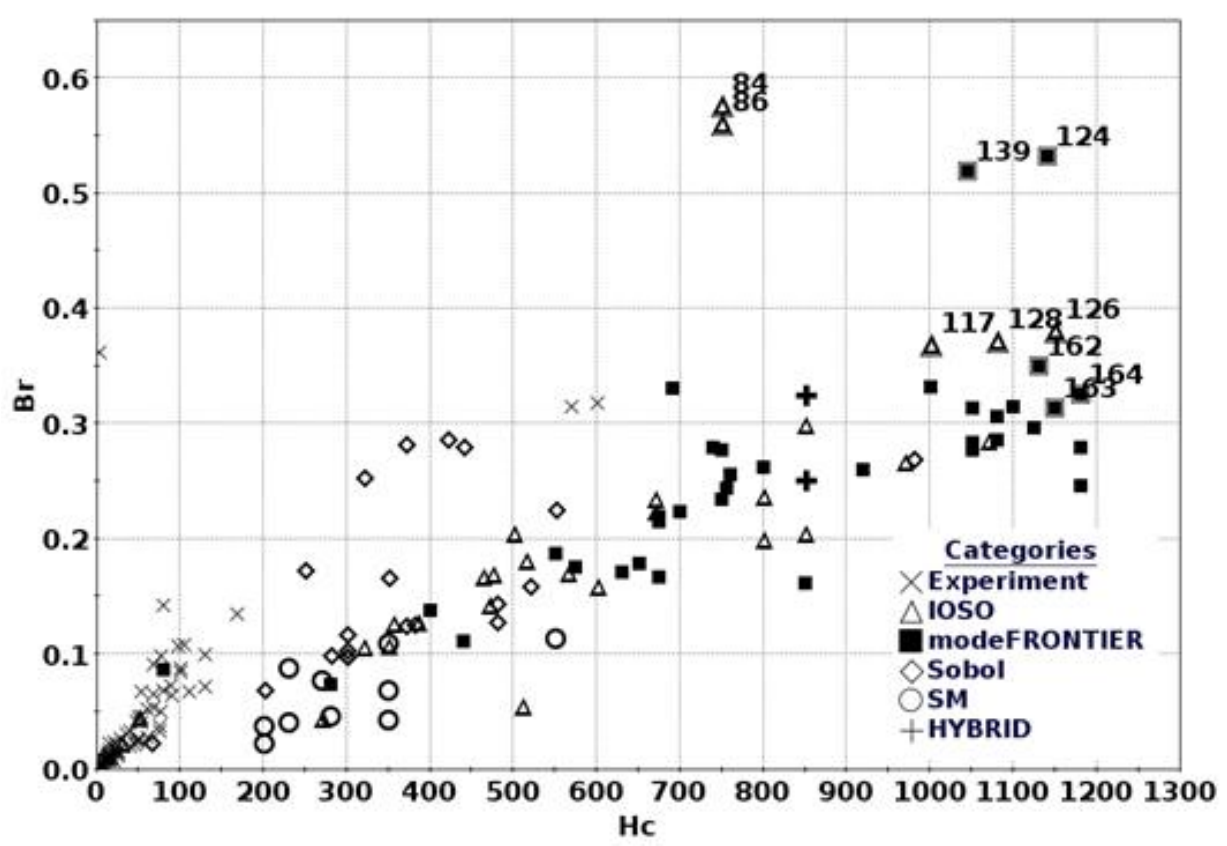

Figure 7: Magnetic coercivity vs magnetic remanence, comparison of solutions by various approaches

It must be noted that all of these alloys were exposed to an identical thermomagnetic protocol. This further demonstrates the efficacy of our approach in handling complex problems of materials design (Jha et al., 2016).

\subsubsection{Alloy composition and distribution}

One of the key aspects in developing a meta-model is the distribution of support points in the variable space. Hence it is important to look at the distribution of alloying elements in the variable space and their behavior over the cycles.

Figure 8 shows the distribution of iron and cobalt in the variable space. It can be seen that the distribution is very poor in the initial 80 alloys. This could have affected the bulk magnetic properties and also the accuracy of meta-models. But, one can notice that by our approach we were able to significantly improve over the 
properties in the subsequent cycles. In the later stages, the distribution seems to be clustered in a narrow region for improved properties. This region was also observed during clustering analysis (6.1) in Chapter 6.

Fe vs. Co: 180 alloys

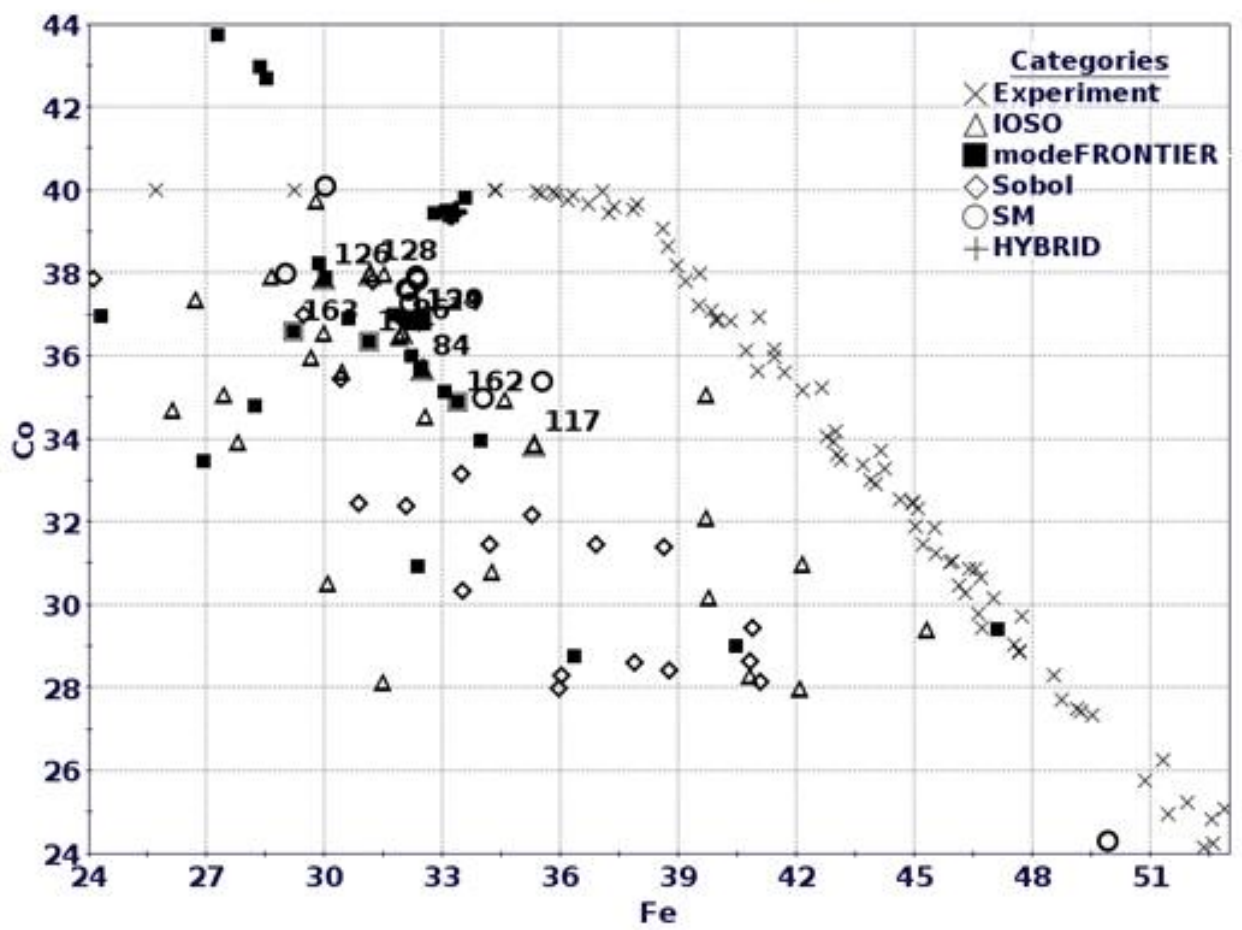

Figure 8: Scatter: Distribution of $\mathrm{Fe}$ and Co concentrations in variable space, comparison of solutions by various approaches

Figure 9 shows the scatter plot between iron and copper in the variable space. Here too, one can observe that the alloys with comparatively superior properties are clustered in a small region. Similar trends can be observed in Figure 10 and Figure 11. In Figure 11, one can clearly observe that the alloys with improved properties are clustered in a region that is far from the initial 80 alloys. 
Fe vs. Cu: 180 alloys

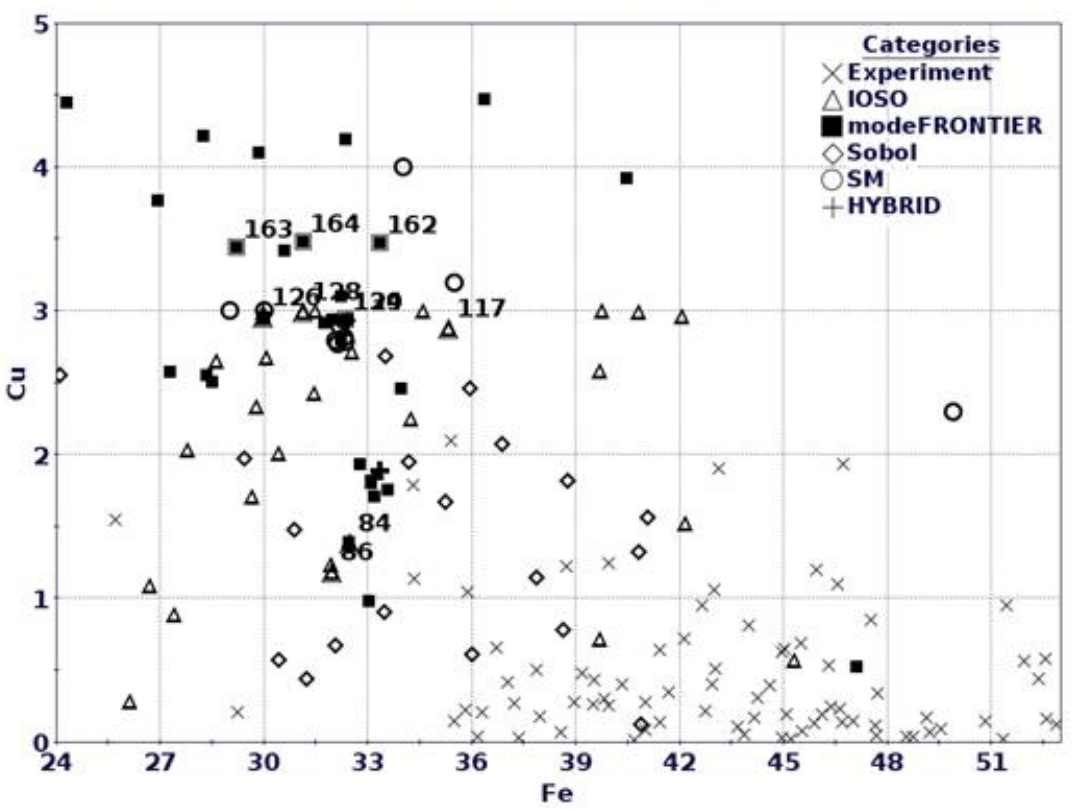

Figure 9: Scatter: Distribution of $\mathrm{Fe}$ and $\mathrm{Cu}$ concentrations in variable space, comparison of solutions by various approaches

Ni vs. Al: 180 alloys

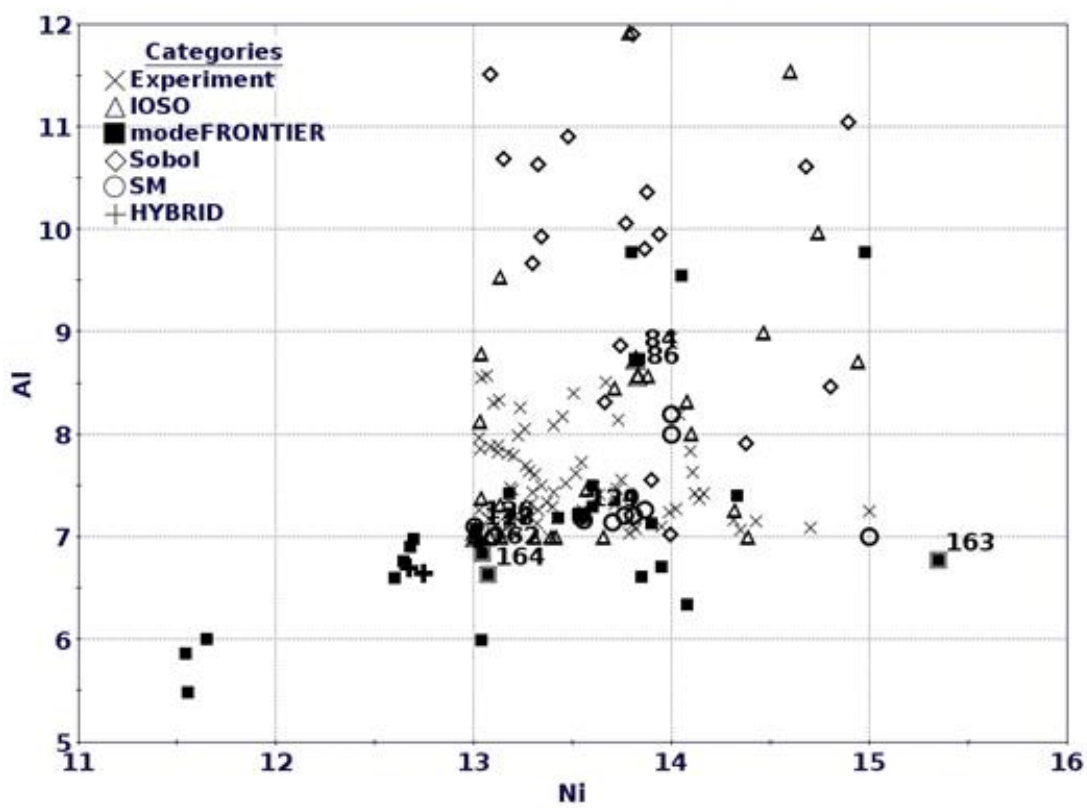

Figure 10: Scatter: Distribution of $\mathrm{Ni}$ and $\mathrm{Al}$ concentrations in variable space; comparison of solutions by various approaches 
Hf vs. Cu: 180 alloys

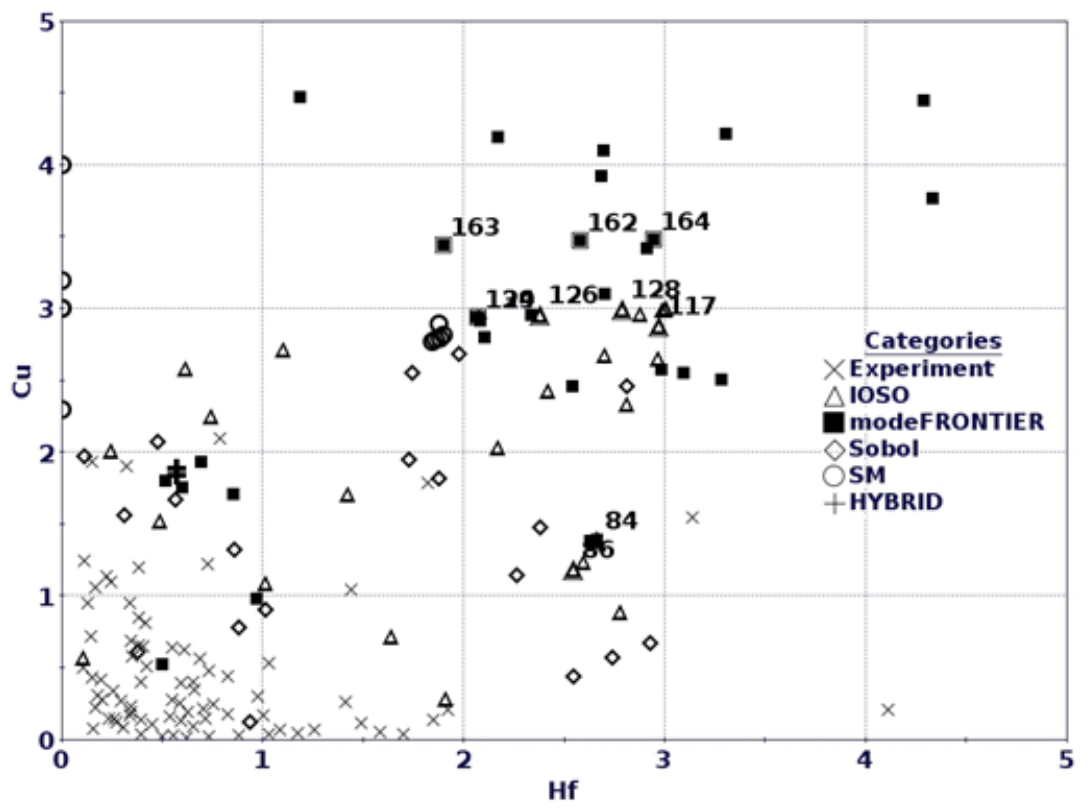

Figure 11: Scatter: Distribution of $\mathrm{Hf}$ and $\mathrm{Cu}$ concentrations in variable space; comparison of solutions by various approaches

Figure 5-7 shows that the alloys predicted by meta-modeling and multiobjective optimization dominate the ones predicted by the Sobol's algorithm (experimental). Figure 8-10 shows clustering of alloys with superior properties. At this point of time, it was also important for us to figure out the element that can be eliminated so as to make room for ree additions. Hence, we processed our data using a set of unsupervised learning algorithms to look for patterns, clusters so that we can proceed further.

\subsection{Meta-model selection}

As discussed in Chapter 3, we have used several approaches to develop metamodels for targeted properties. Meta-models were examined on the basis of accuracy measures listed in Chapter 4 and the most accurate meta-models have been listed in Table 5. 
Table 5: Meta-models selected for targeted objectives

\begin{tabular}{|l|l|}
\hline Properties & Response Surface \\
\hline$(B H)_{\max }$ & $\mathrm{RBF}($ Gaussian $)$ \\
\hline$H_{c}$ & $\mathrm{RBF}(\mathrm{MR})$ \\
\hline$B_{r}$ & $\mathrm{RBF}(\mathrm{IMQ})$ \\
\hline$M_{s}$ & $\mathrm{ED}$ \\
\hline$M_{r}$ & $\mathrm{RBF}(\mathrm{IMQ})$ \\
\hline$(B H)_{\max } /$ mass & $\mathrm{akr}(\mathrm{Gaussian})$ \\
\hline magnetic permeability & $\mathrm{RBF}(\mathrm{IMQ})$ \\
\hline cost of raw materials & $\mathrm{RBF}(\mathrm{MQ})$ \\
\hline$j H_{c}$ & $\mathrm{AKR}(\mathrm{Gaussian})$ \\
\hline density & $\mathrm{RBF}(\mathrm{MQ})$ \\
\hline
\end{tabular}

\subsubsection{SVR analysis for selected models}

One of the selection criteria for a meta-model was its ability to mimic information on the composition-property from the literature. SVR analysis was performed for all the selected meta-models mentioned in Table 5 and the results are tabulated in Table 6 (Jha et al., 2016), (Jha et al., 2016a). 
Table 6: Single variable response for various objectives

\begin{tabular}{|c|c|c|c|c|c|c|c|c|c|}
\hline \multirow{2}{*}{$\begin{array}{c}\text { Objective } \\
\text { no. }\end{array}$} & \multirow{2}{*}{ Objectives } & \multicolumn{8}{|c|}{ Variable response } \\
\hline & & $\mathrm{Fe}$ & Co & $\mathrm{Ni}$ & $\mathrm{Al}$ & $\mathrm{Ti}$ & $\mathrm{Hf}$ & $\mathrm{Cu}$ & $\mathrm{Nb}$ \\
\hline 1 & $(B H)_{\max }$ & Nil & Nil & Mix & Nil & Nil & Nil & Nil & Nil \\
\hline 2 & $H_{c}$ & Mix & Mix & Mix & Inv & Mix & Dir & Dir & Mix \\
\hline 3 & $B_{r}$ & Mix & Mix & Mix & Inv & Mix & Dir & Dir & Inv \\
\hline 4 & $M_{s}$ & Dir & $\operatorname{lnv}$ & Dir & Mix & Inv & Dir & Mix & Mix \\
\hline 5 & $M_{r}$ & Nil & Nil & Nil & Nil & Nil & Nil & Nil & Nil \\
\hline 6 & $(B H)_{\max } /$ mass & Nil & Nil & Nil & Nil & Nil & Nil & Nil & Nil \\
\hline 7 & $\begin{array}{l}\text { Magnet } \\
\text { permeability }\end{array}$ & Mix & Mix & Mix & Mix & Inv & Mix & Mix & Mix \\
\hline 8 & $\begin{array}{l}\text { cost of raw } \\
\text { material }\end{array}$ & $\operatorname{lnv}$ & $\ln v$ & $\operatorname{lnv}$ & Dir & Dir & Dir & $\operatorname{lnv}$ & Dir \\
\hline 9 & $j H_{c}$ & Mix & Mix & Mix & $\operatorname{lnv}$ & $\operatorname{lnv}$ & Mix & Dir & Mix \\
\hline 10 & density & Mix & Dir & $\operatorname{Mix}$ & $\operatorname{lnv}$ & $\operatorname{lnv}$ & Mix & Mix & Dir \\
\hline
\end{tabular}

Current experimental dataset was quite noisy. So, we were left with a lot of mixed responses. A few important findings can be listed as follows:

1. Copper shows a direct response for $H_{c}$ and $B_{r}$, thus response surface predictions are at par with available literature (Dilon, 2014). This has been discussed earlier in Chapter 2. 
2. Hafnium shows a direct response for $H_{c}$ and $B_{r}$. Hf has not been previously used in AINiCo alloys. Hence, further data-analysis is required before reaching a final conclusion.

3. Nickel shows mixed response with $(B H)_{\max }, H_{c}$ and $B_{r}$ as can be seen from Figures 12,13 and 14, respectively. While it shows positive response for $M_{s}$ as can be seen from Figure 15.

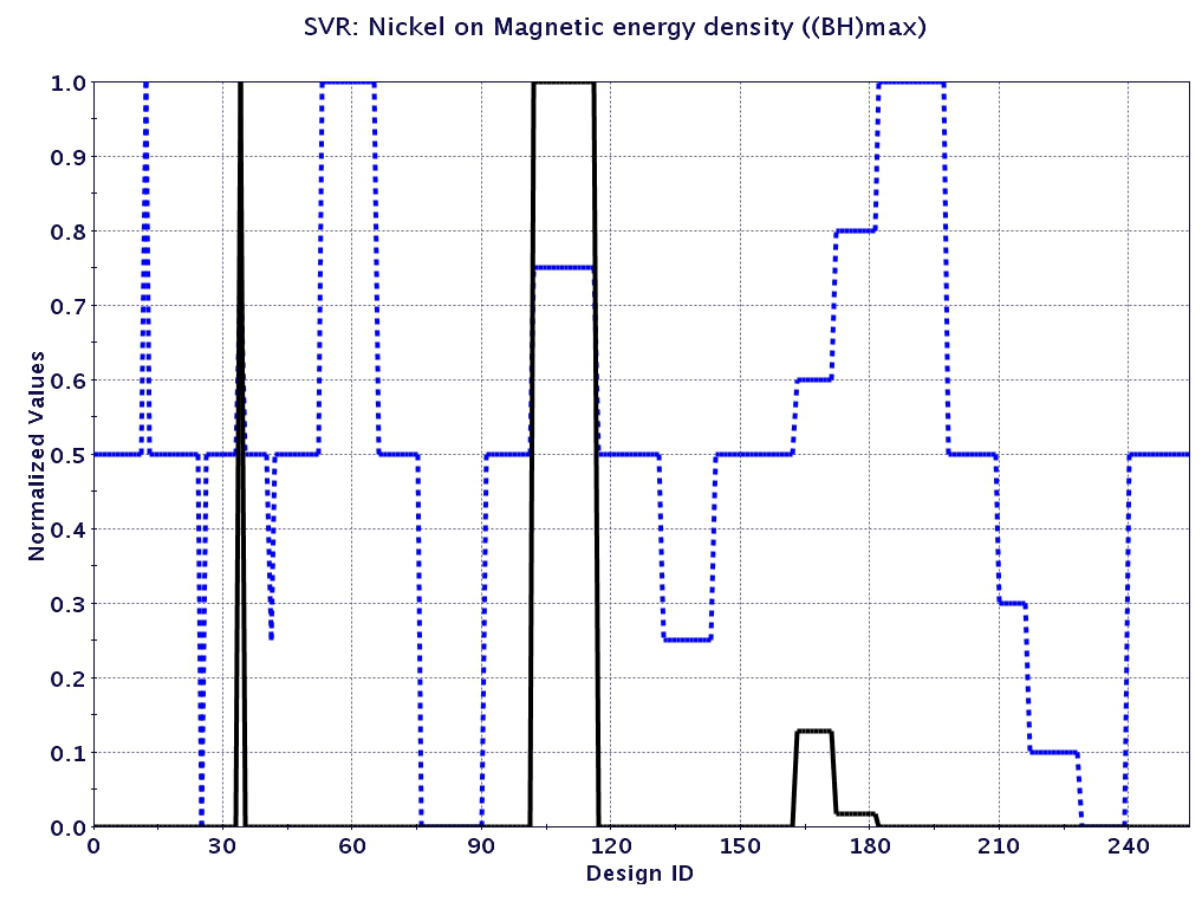

Figure 12: SVR: Nickel on magnetic energy density $(B H)_{\max }$ 
SVR: Nickel on Magnetic coercivity $(\mathrm{Hc})$

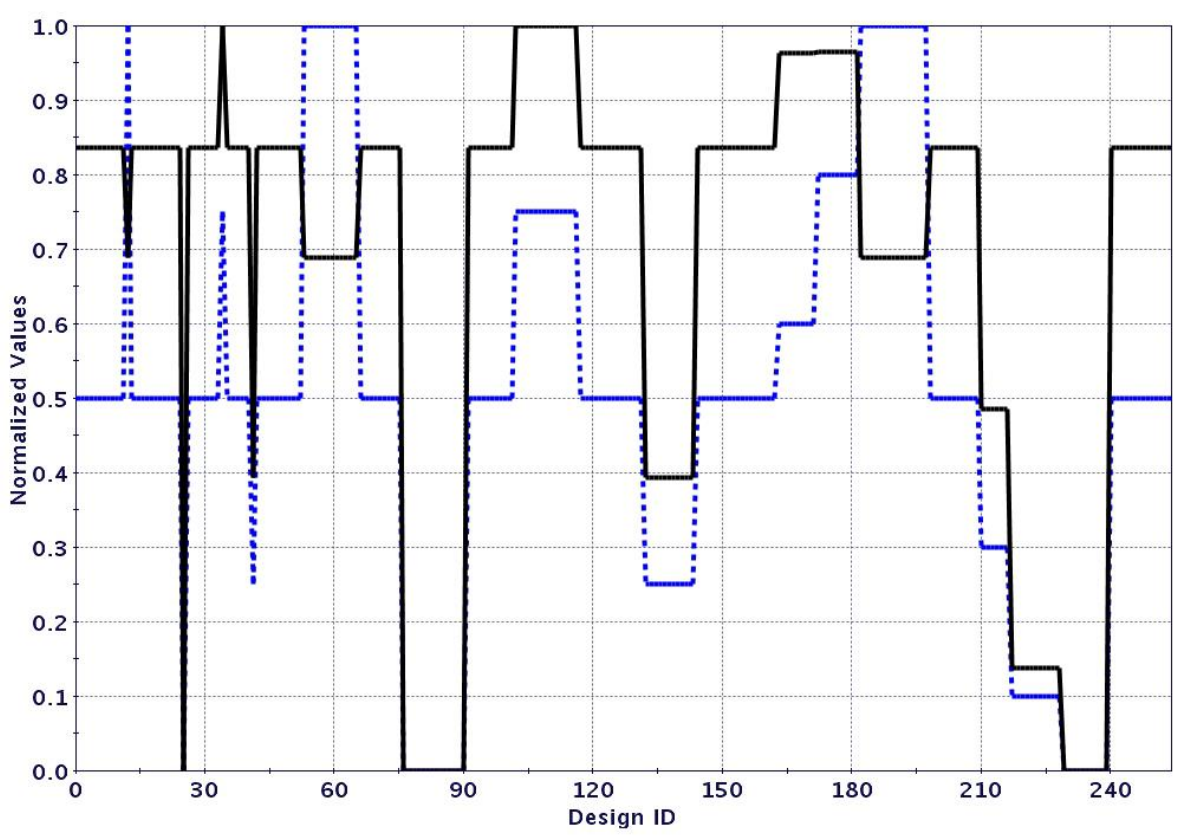

Figure 13: SVR: Nickel on magnetic Coercivity $\left(H_{c}\right)$

SVR: Nickel on Magnetic remanence $(\mathrm{Br})$

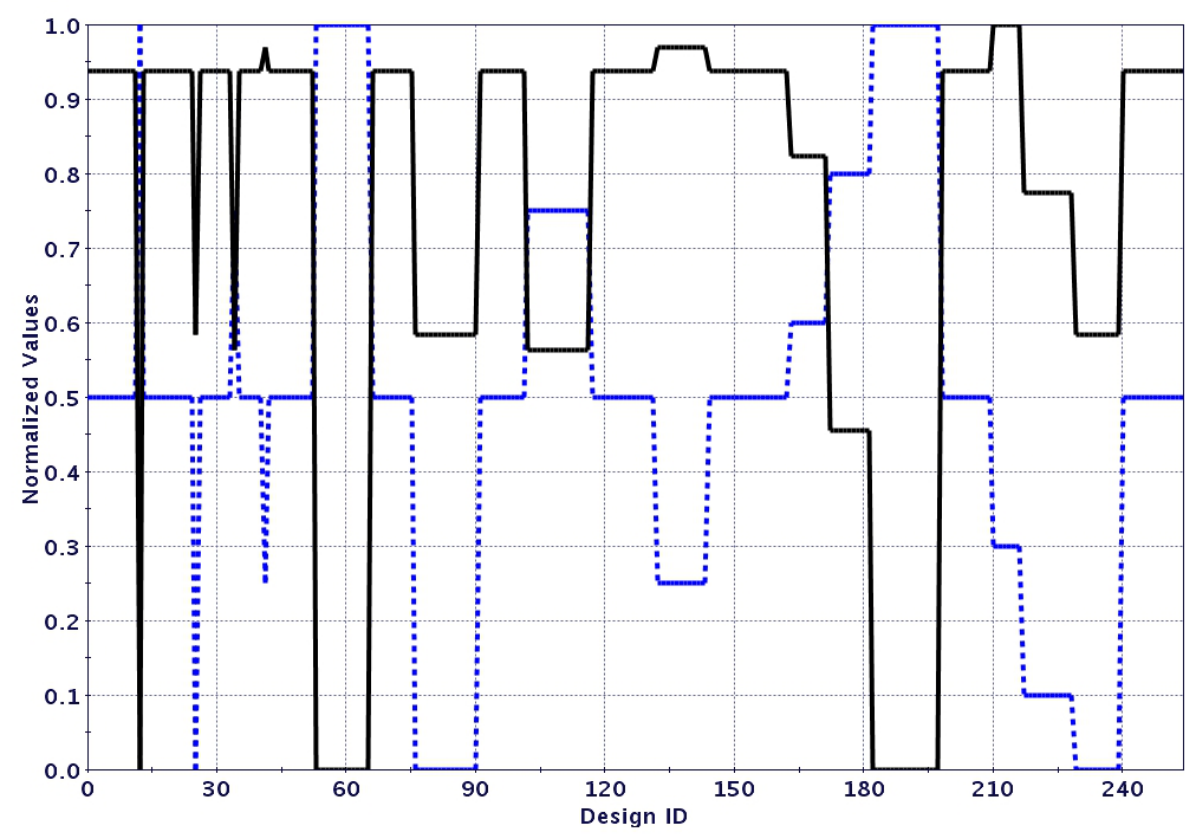

Figure 14: SVR: Nickel on magnetic remannence $\left(B_{r}\right)$ 
SVR: Nickel on Saturation magnetization (Ms)

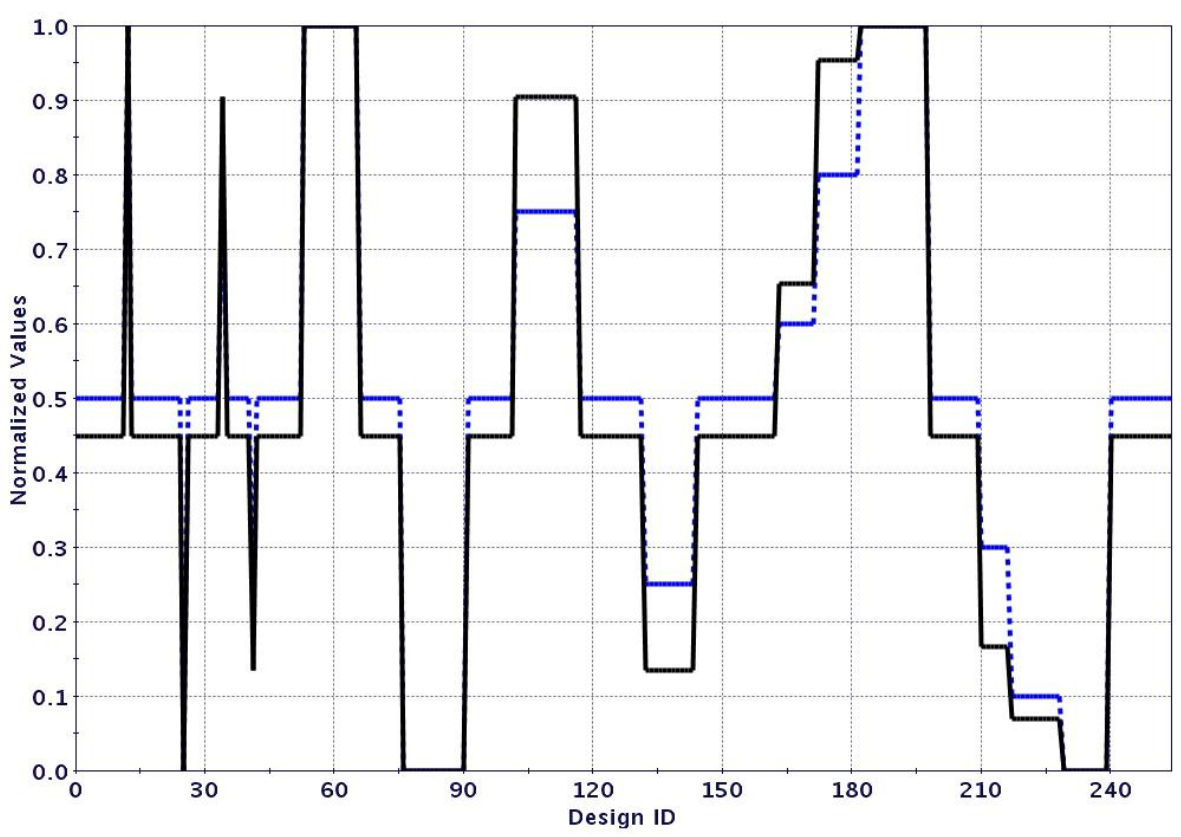

Figure 15: SVR: Nickel on saturation magnetization $\left(M_{s}\right)$

At this point, we are left with a few responses that is similar to those reported in the literature in Chapter 2. Thus, meta-modeling can prove to be an asset for developing alloys in the future as well as in predicting the properties of alloys with a new composition. We moved forward to use these models for multi-objective optimization according to the problem formulated in Chapter 3. 


\section{CHAPTER 6 RESULTS 2- UNSUPERVISED LEARNING}

\subsection{Heirarchichal Clustering Analysis (HCA)}

We clustered the alloys on the basis of targeted properties. Dendrogram was cut in a manner so that we got a total of nine clusters (cluster 0 to cluster 8) as denoted by the numbers in the dendrogram plot. Figure 16 shows the full dendrogram plot obtained from HCA analysis shows all the 9 clusters.

In a later analysis, cluster 8 and cluster 7 were merged as one when analyzed by Ward's approach (Ward, 1963). Clustering parameters and the number of alloys included in each cluster has been tabulated in Table 7. Figure 17 shows a simplified dendrogram plot obtained from HCA analysis, which is clearer for viewing different clusters and contains 8 clusters, as mentioned in Table 7 (Jha et al., 2016).

HCA: 180 alloys

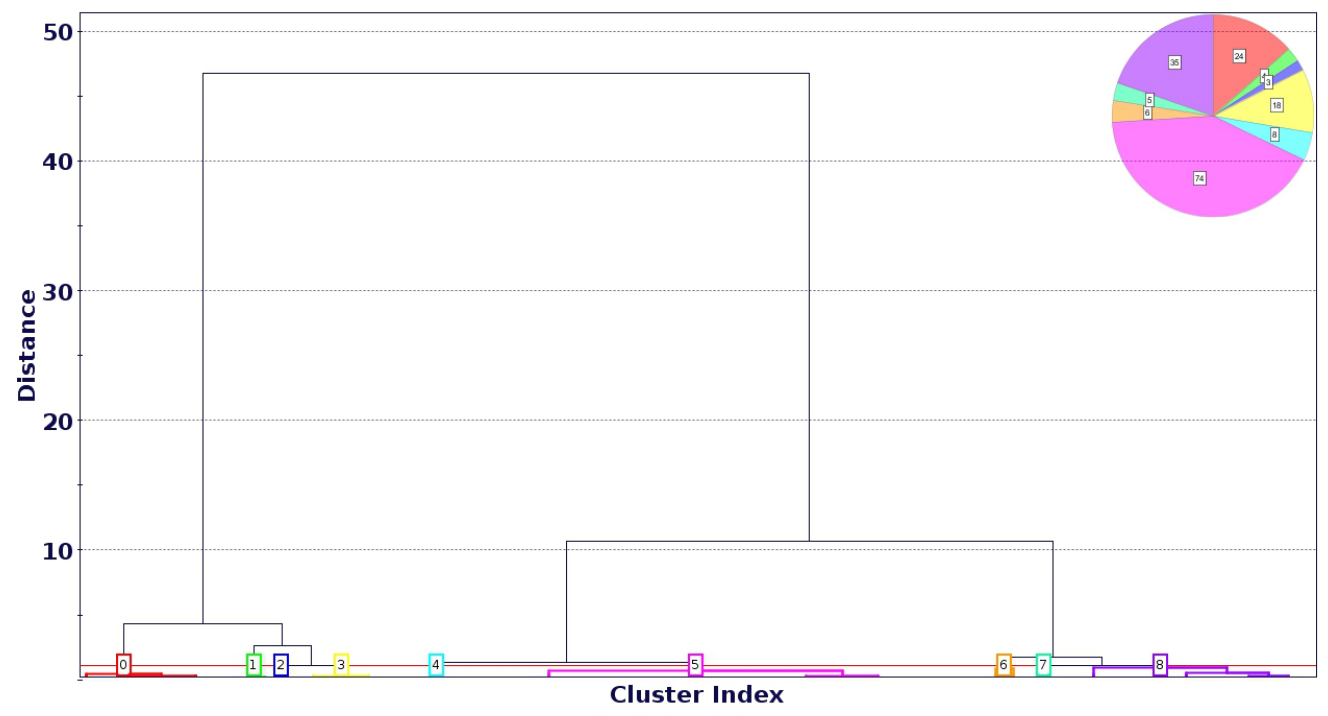

Figure 16: Dedrogram plot from HCA analysis 
Table 7: Clustering parameters in HCA analysis

\begin{tabular}{|c|c|c|c|c|}
\hline Cluster no. & Cluster Size & ISim & ESim & Best alloy \\
\hline 0 & 24 & 2.5 & 1.1 & 175,115 \\
\hline 1 & 4 & 1.5 & 0.6 & $84,86,124,139$ \\
\hline 2 & 3 & 1.5 & 0.7 & $145,146,147$ \\
\hline 3 & 18 & 3.2 & 0.8 & $117,126,128$ \\
\hline 4 & 8 & 4.5 & 1.3 & \\
\hline 5 & 74 & 4.6 & 1.0 & \\
\hline 6 & 6 & 1.7 & 1.0 & \\
\hline 7 & 40 & 2.1 & 1.3 & \\
\hline
\end{tabular}

HCA: 180 alloys

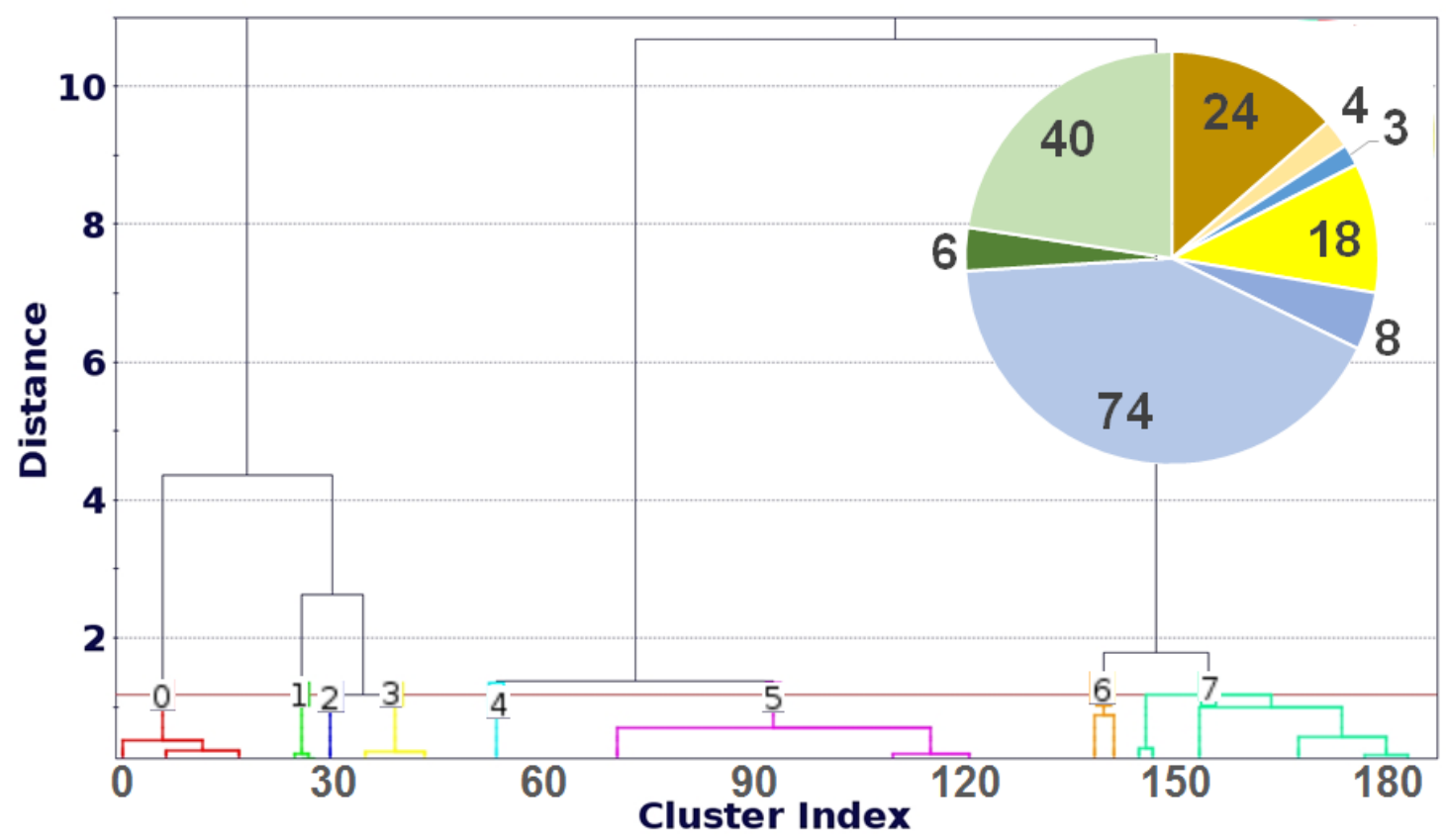

Figure 17: Simplified dedrogram plot from HCA analysis 
From Table 7, it can be seen that cluster 1 , cluster 2 and cluster 3 have higher Isim, while a lower Esim when compared to other clusters. Cluster 1 and cluster 3 contains candidates from top 10 alloys based on $(B H)_{\max }$ value, while alloys in cluster 2 posses high $H_{c}$. Hence, we focussed on cluster 1, 2 and 3 for determining composition-property relationship in HCA analysis.

HCA analysis findings were used to crosscheck the findings from SVR analysis mentioned in section 5.2.1. Following text includes cluster scatter plots for various elements vs $(B H)_{\max }, H_{c}$ and $B_{r}$. In the following figures, the confidence level for both the confidence interval and confidence ellipse was set at 0.9. These figures proved to be helpful in determining the variable bounds for targeted properties.

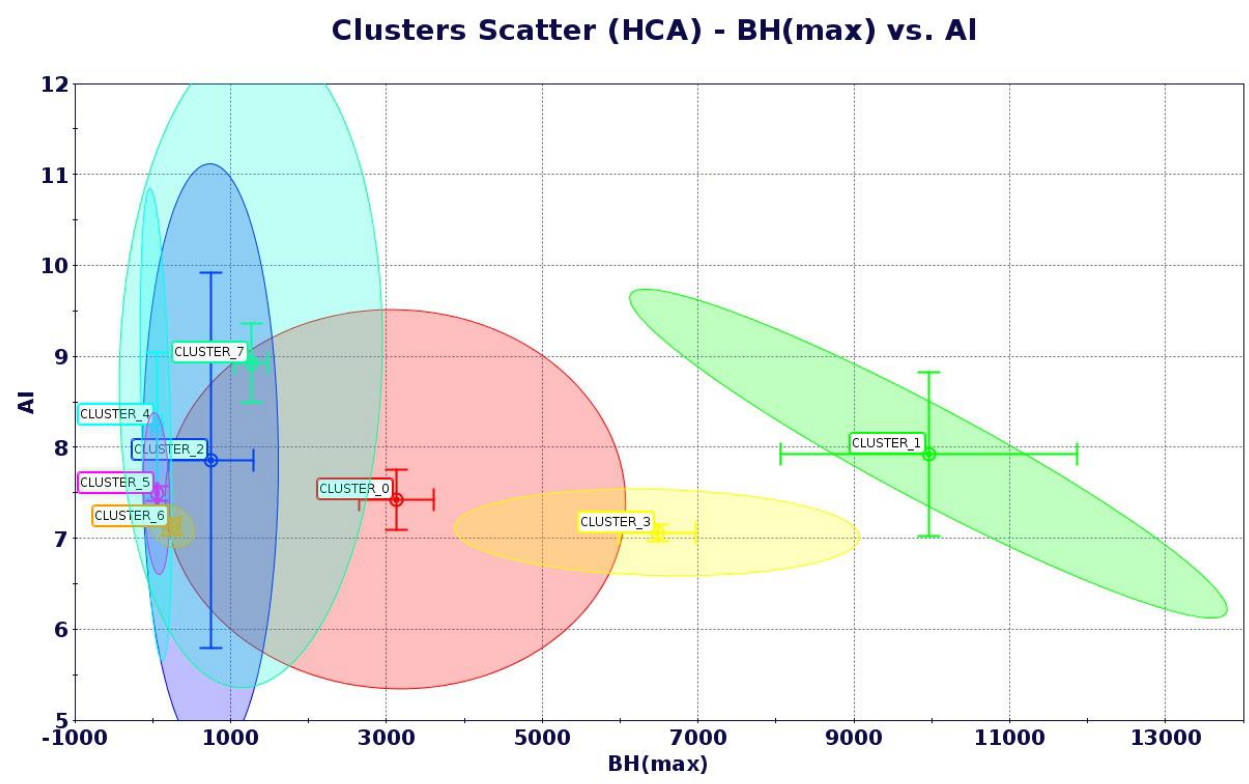

Figure 18: Clusters scatter: $(B H)_{\max }$ vs Aluminum 
In Figure 18, for cluster 1, $(B H)_{\max }$ increases with decrease in Aluminum content in the range $6-10 \mathrm{wt} \%$. For cluster $3,(B H)_{\max }$ varies with Aluminum content in a very narrow range around $7 \mathrm{wt} \%$. Apart from that, we cannot draw any meaningful conclusion from other clusters.

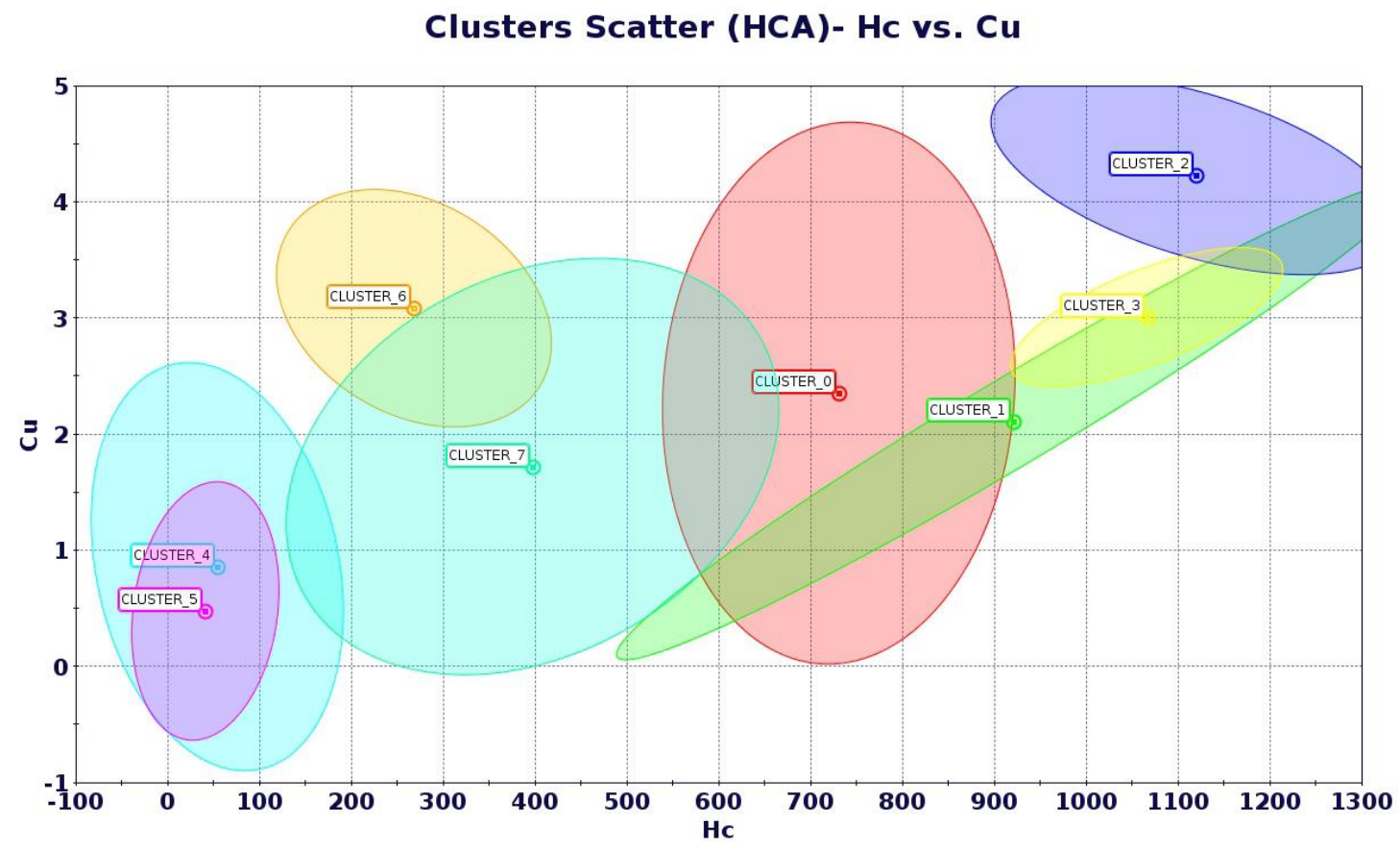

Figure 19: Cluster scatter: $H_{c}$ vs Copper

In Figure 19, it can be observed that that $H_{c}$ increases with an increase in Cu content in cluster 1 and Cluster 3. While $H_{c}$ decreases with increasing $\mathrm{Cu}$ content in cluster 2 in a narrow composition range. Additionally, it can be seen that the three clusters overlap between 3-5 wt \% Cu. Overall, copper affects $H_{c}$ 
positively, as reported in the literature (Section 2) as well as SVR analysis (5.2.1). Hence, optimum copper concentration must be maintained between 3-5 wt\%. Apart from that, we cannot draw any meaningful conclusion from other clusters.

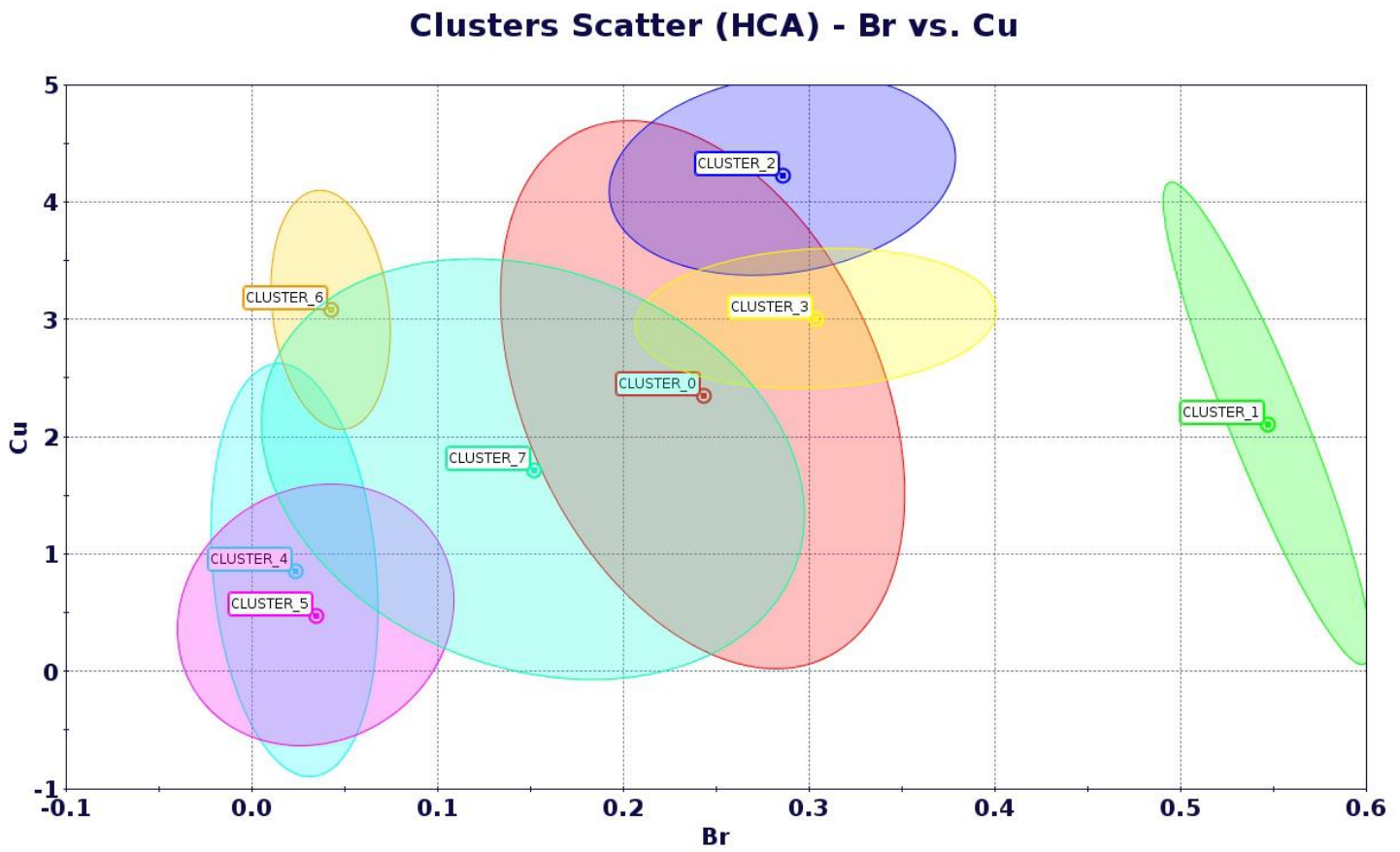

Figure 20: Cluster scatter: $B_{r}$ vs Copper

In Figure 20, cluster 1 shows a slight variation of $B_{r}$ over a wide range of copper concentration ( 0 - 4\%), while for cluster $3, B_{r}$ varies in a narrow range of copper concentration at about $3 \mathrm{wt} \%$. From these results, it is difficult to determine the role of $\mathrm{Cu}$ addition to $B_{r}$. From the literature (Section 2) as well as SVR analysis (section 5.2.1), Cu tends to affect $B_{r}$ positively. Hence, this needs further investigation. 


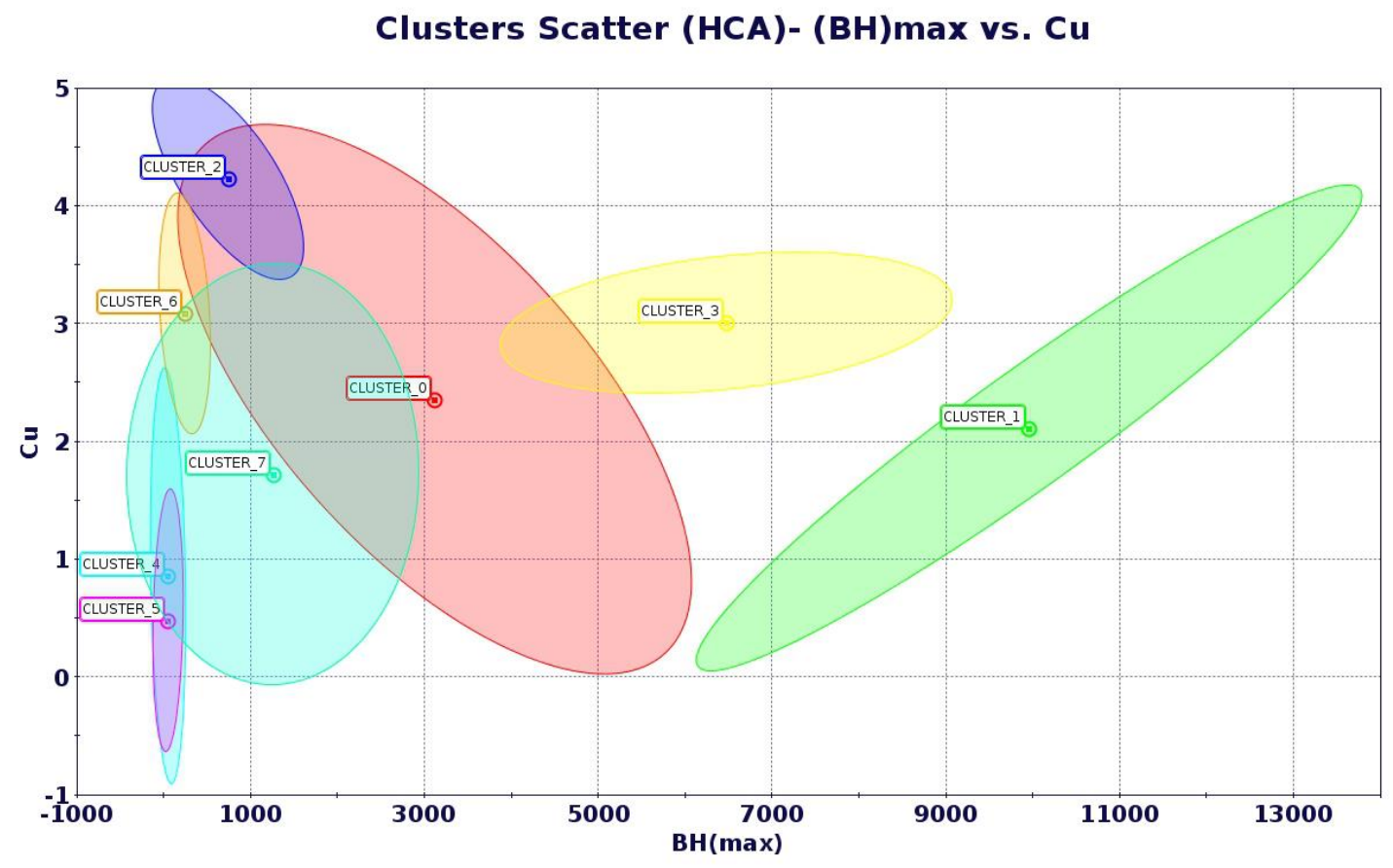

Figure 21: $(B H)_{\max }$ vs Copper

Figure 21 shows a plot for $(B H)_{\max }$ vs Copper for various clusters. In cluster $1,(B H)_{\max }$ increases with an increase in Copper content. The end of confidence ellipse is around $4 \mathrm{wt} \%$ copper. This region (around $4 \mathrm{wt} \% \mathrm{Cu}$ ) was also observed in $H_{c}$ vs Cu plot of $\mathrm{HCA}$, and it seems to be helpful for improving upon $H_{c}$ as can be seen in Figure 19. $B_{r}$ and $H_{c}$ are conflicting (Figure 4), and it can also be observed from Figure 20 that there is a slight decrease in value of $B_{r}$ in cluster 1 at around $4 \mathrm{wt} \% \mathrm{Cu}$. Hence, optimum Copper content must be around $4 \mathrm{wt} \%$ for improvement in $(B H)_{\max }$ and $B_{r}$. 


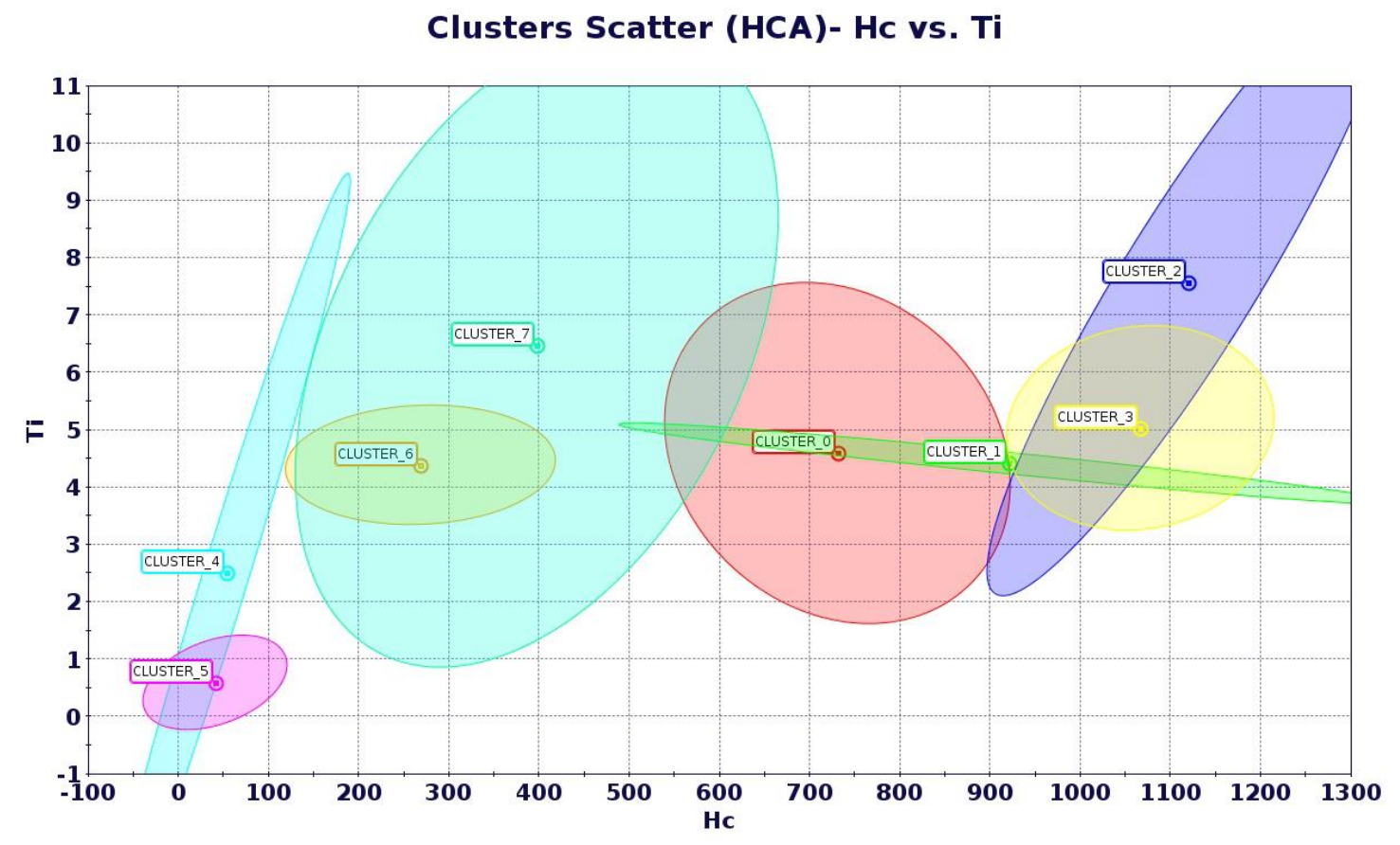

Figure 22: $H_{c}$ vs Titanium

In Figure 22, for cluster $7, H_{c}$ tends to increase wiith an increase in $\mathrm{Ti}$ content. For cluster 1 , one can observe that $H_{c}$ tends to increase in a very narrow range of copper concentration of about 4 - $5 \mathrm{wt} \%$. From the literature (section 2), $\mathrm{Ti}$ tends to increase $H_{c}$, but at the expense of $B_{r}$. Hence, from the present analysis, optimum Ti content appears to be around $4 \mathrm{wt} \%$. 
Clusters Scatter (HCA)- BH(max) vs. Fe

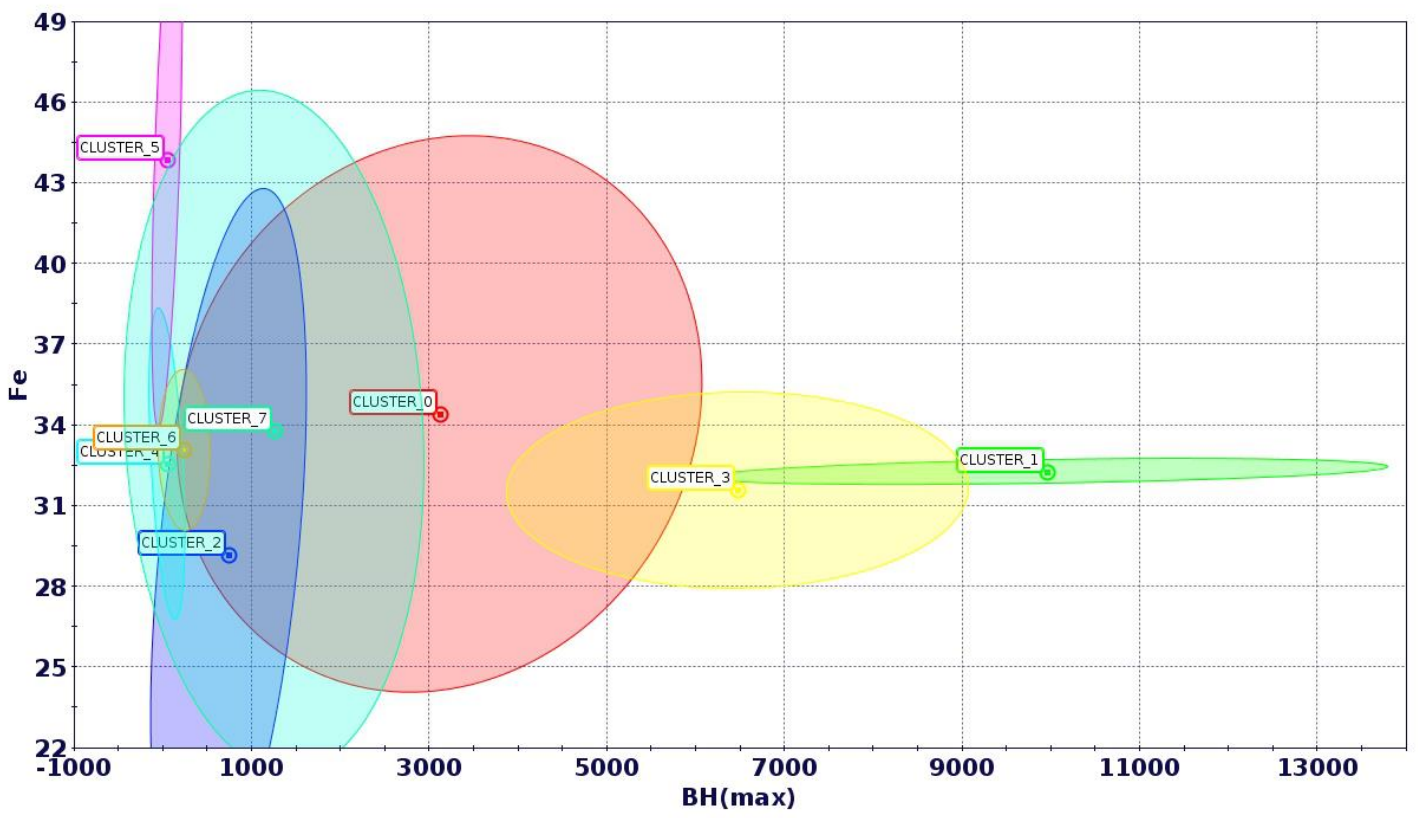

Figure 23: $(B H)_{\max }$ vs Iron

In Figure 23, it can be seen from cluster 1, that in order to increase $(B H)_{\max }$ , one needs to stay in a narrow range for Iron at about $32 \mathrm{wt} \%$. 
Clusters Scatter (HCA)- BHmax vs. Ni

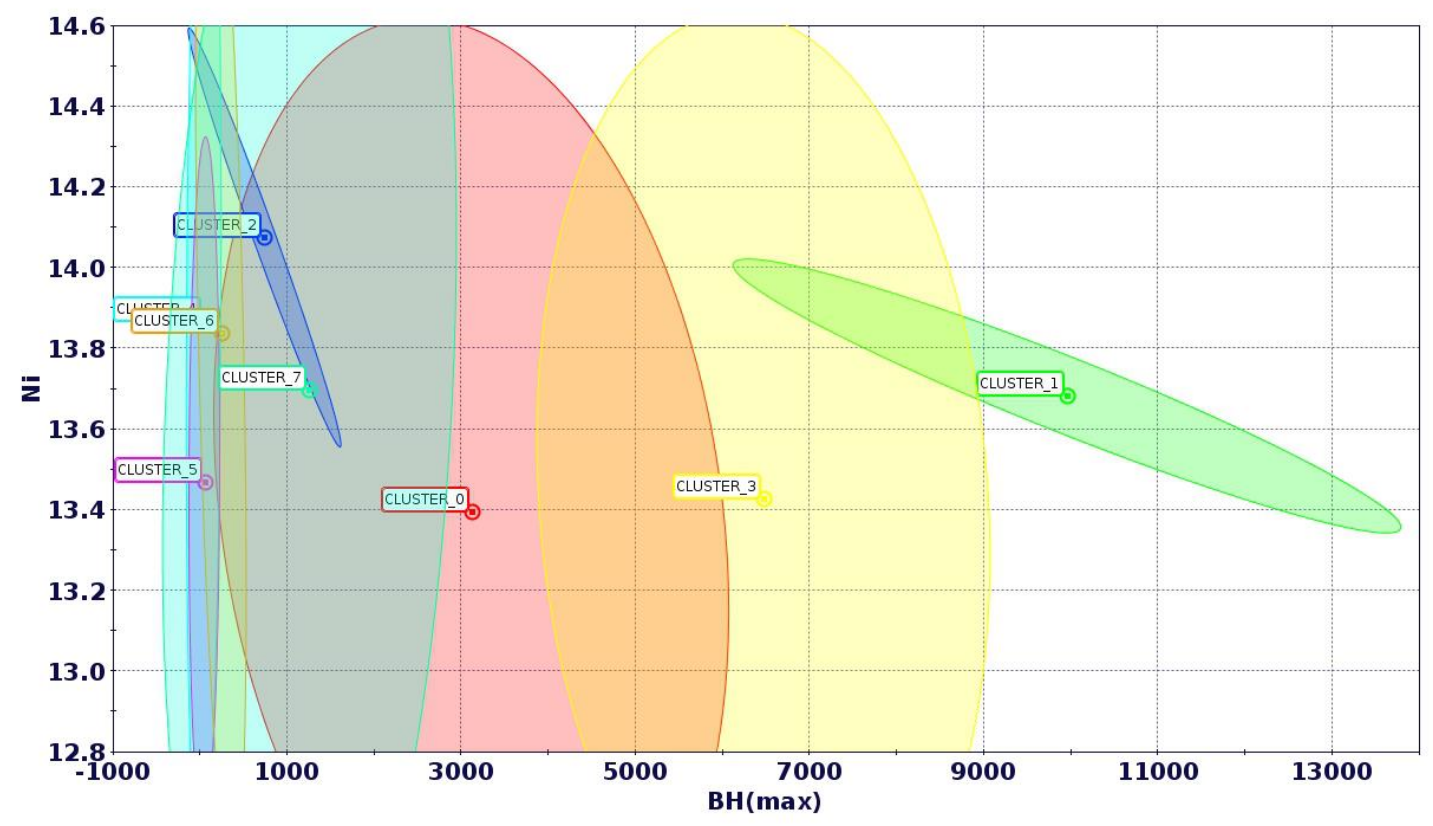

Figure 24: Clusters scatter: $(B H)_{\max }$ vs Nickel

In Figure 24, Nickel shows a weak response for $(B H)_{\max }$ (also in the SVR analysis, Section 5.2.1). In cluster $1,(B H)_{\max }$ increases with decrease in $\mathrm{Ni}$ content in a very narrow range of composition (13.4 - $14 \mathrm{wt} \%)$.

One can also use these plots for discarding a few elements in order to make way for a rare - earth addition. We plotted scatter plots for Niobium vs $(B H)_{\max }$, $H_{c}$ and $B_{r}$. 


\section{Clusters Scatter (HCA)- Hc vs. Nb}

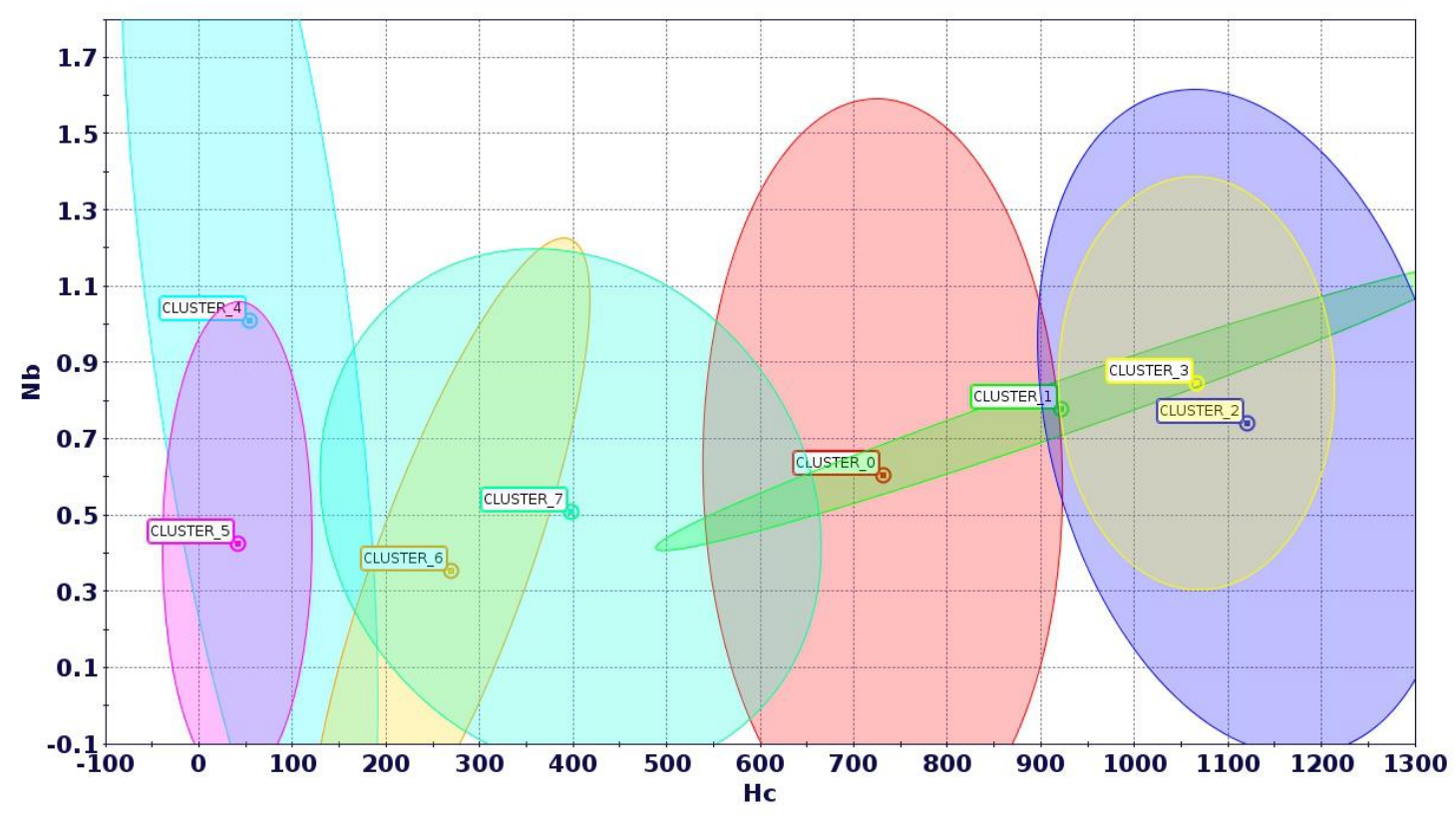

Figure 25: Clusters scatter: $H_{c}$ vs. Niobium

\section{Clusters Scatter (HCA)- Br vs. Nb}

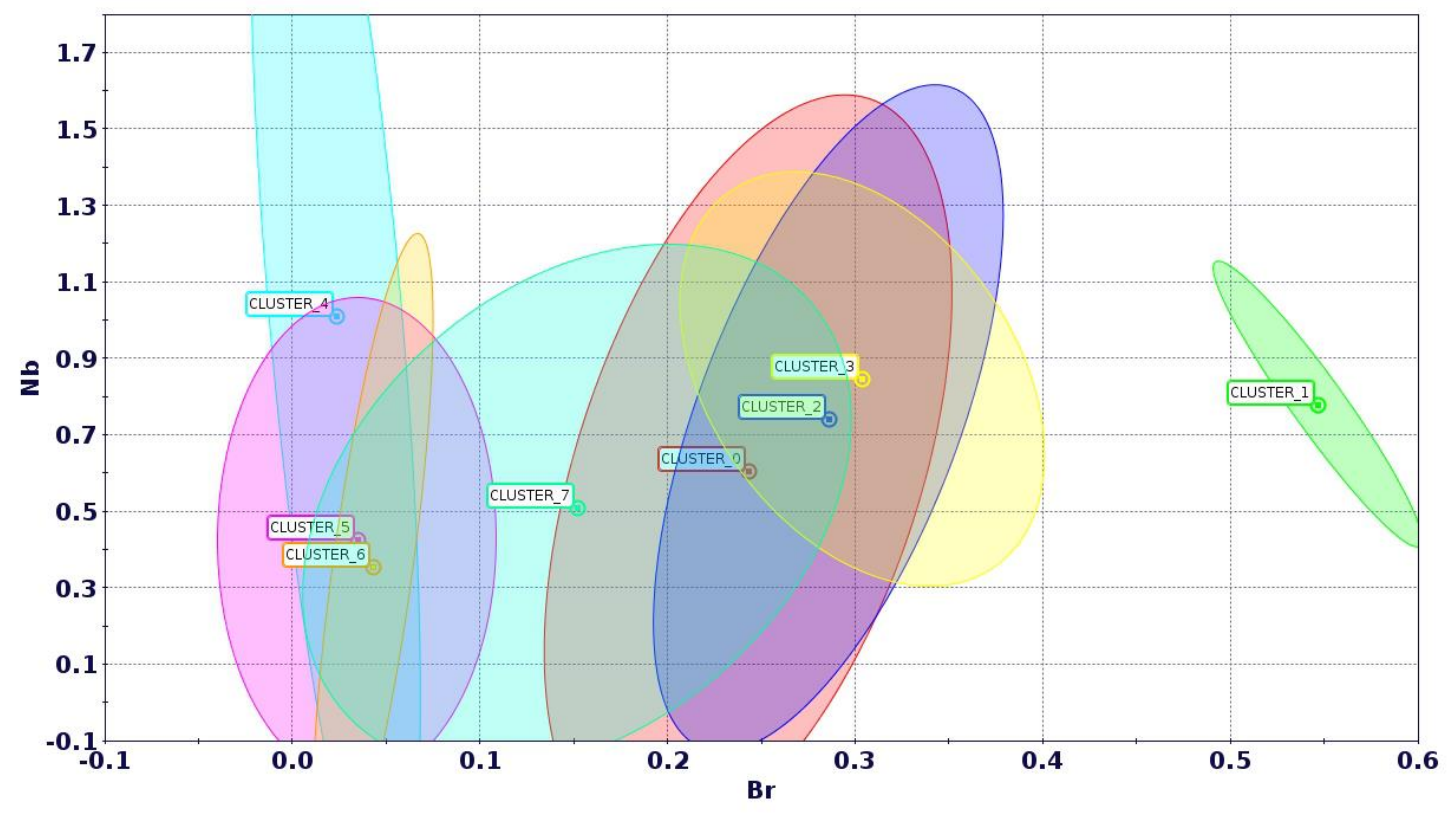

Figure 26: Clusters scatter: $B_{r}$ vs. Niobium 


\section{Clusters Scatter (HCA)- BH(max) vs. Nb}

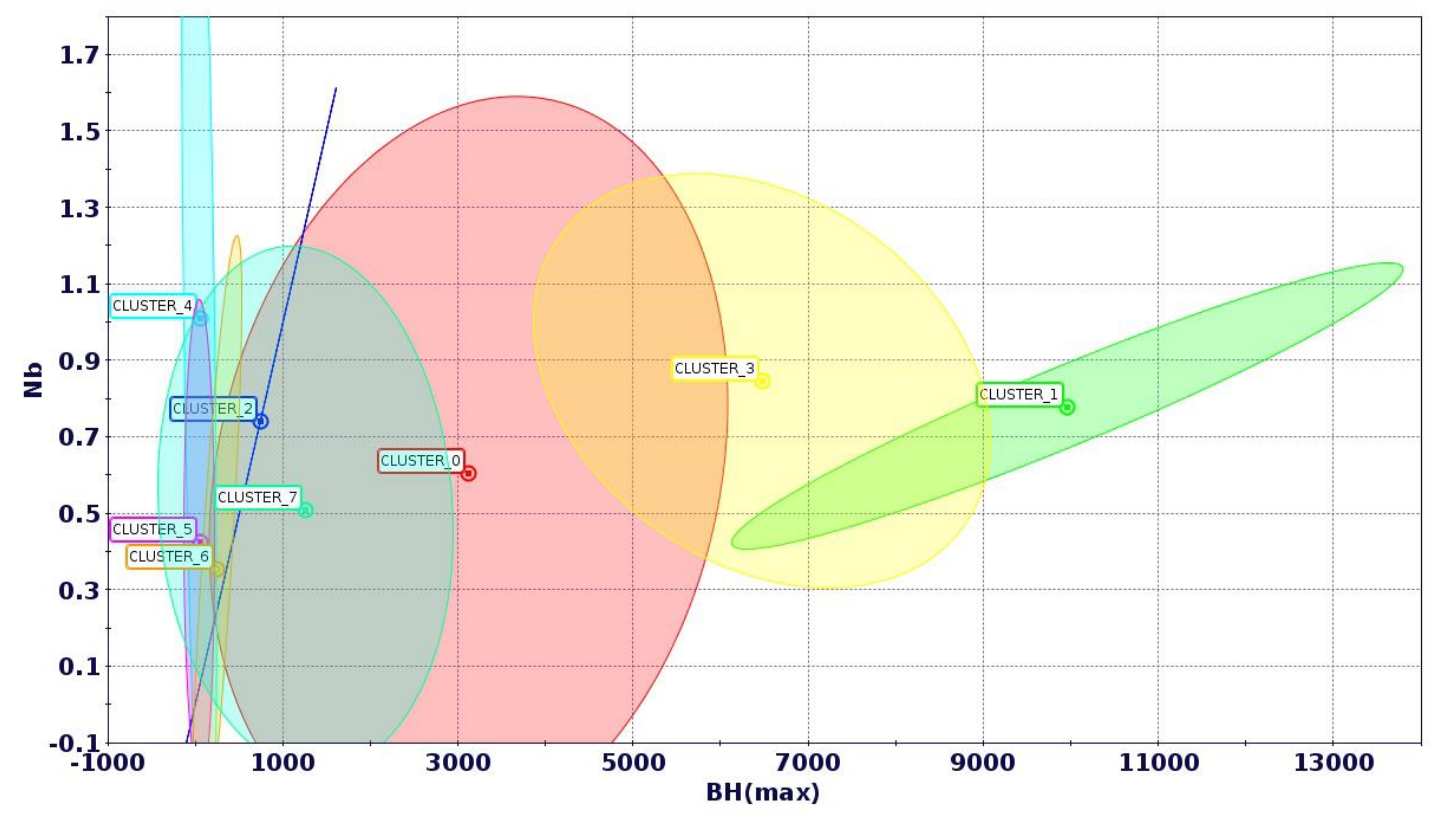

Figure 27: Clusters scatter: $(B H)_{\max }$ vs. Niobium

From Figures 25, 26 and 27, one can see that Niobium has almost no influence on $H_{c}$ and $B_{r}$. This was also observed in the SVR analysis (Section 5.2.1). Additionally, Niobium has the same effect as Titanium (Chapter 2). Hence, one can think of manufacturing a few samples without $\mathrm{Nb}$. Or, $\mathrm{Nb}$ can be replaced with a rare-earth element.

\subsection{Principal Component Analysis (PCA)}

The alloys were also plotted along with the elements and its orientations. Here, the alloys were clustered by K-means clustering method to classify the alloys into different clusters. Alloys that belong to the same cluster have the same symbol. Few best alloys mentioned in Table 2 were plotted in the figure. In these 
figures, variables (elements) were plotted as arrows. The arrows represent the relative contribution of the original variables to the variance along the PCs. In these figures, the longer the arrows, the stronger are their contributions. Additionally, an arrow orthogonal to a certain PC has a null effect on that PC while an arrow that is collinear to a certain PC contribute only to that certain PC.

We classified the dataset into four sets and performed the PC analysis on individual sets in order to extract information from one set and then cross check it with the findings of other sets. In all of these cases, PC1, PC2, and PC3 were able to capture most of the variance of the dataset. The data set was classified as follows:

1. Experimental: Alloy 1-80

2. Optimization: Alloy $81-180$

3. Data categorized based on Multi-Criterion Decision Making (MCDM): 40 alloys were selected.

4. Whole dataset: Alloy 1-180.

We used a popular statistical software, IBM SPSS (IBMSPSS, 2015), and Multivariate Data Analysis (MVA) node in optimization package modeFRONTIER (ESTECO, 2015) for this work (Jha et al., 2016a).

1. Experimental: Alloy 1-80:

These were the initial set of compositions predicted by Sobolâ€ $\epsilon^{\mathrm{TM}} \mathbf{S}$ algorithm. Thus, we did not perform PCA on the elements. Various properties were analyzed and it is reported below. Scree plots were plotted in order to determine the number of effective principal components required to represent the whole data 
set. It was found that 2 PC's are able to extract most of the information from the dataset. Figure 28 shows the scree plot for the properties while Figure 29 shows the position of various properties in the PC space.

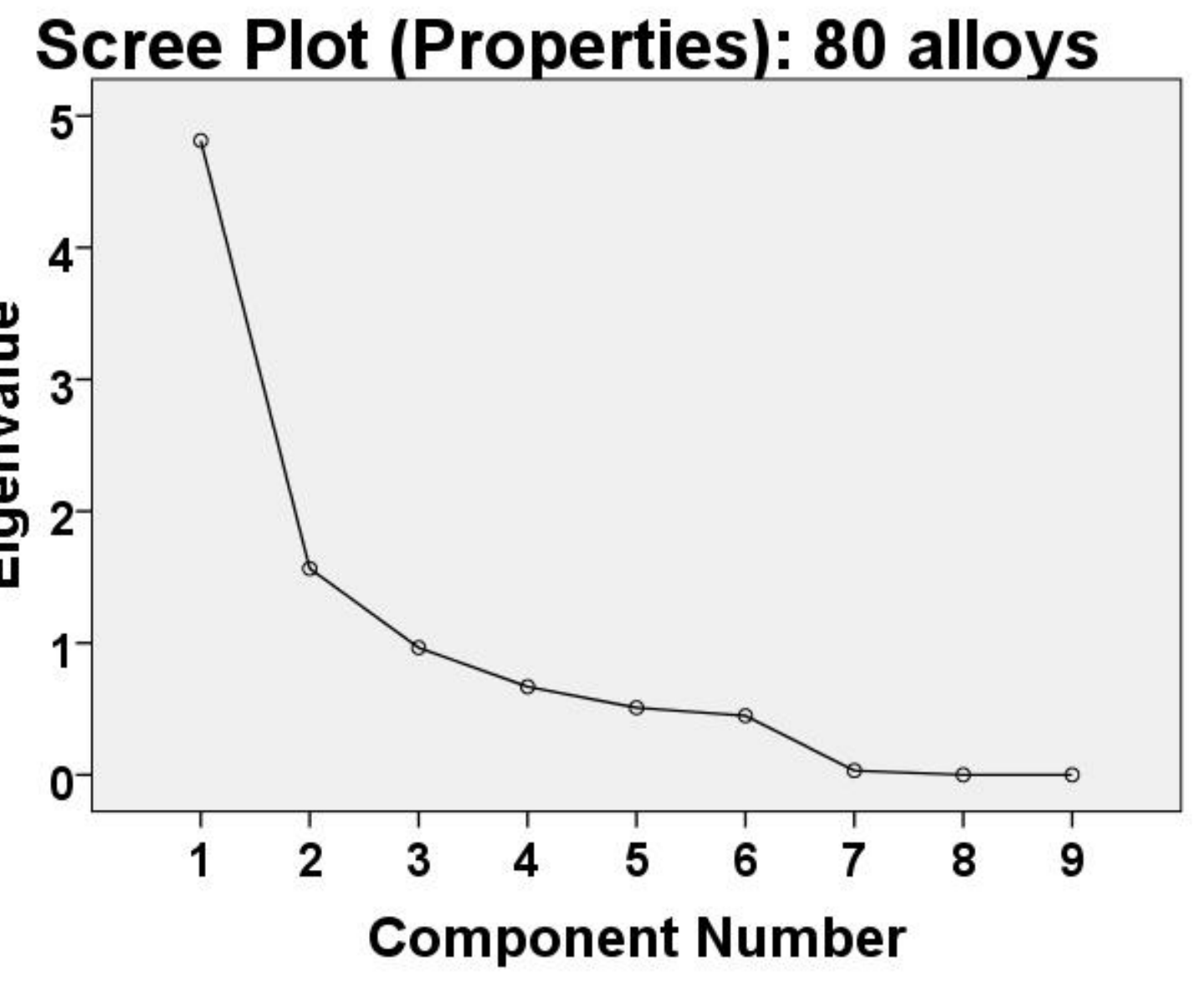

Figure 28: Scree plot for PCA analysis: 2 PCA components were chosen 


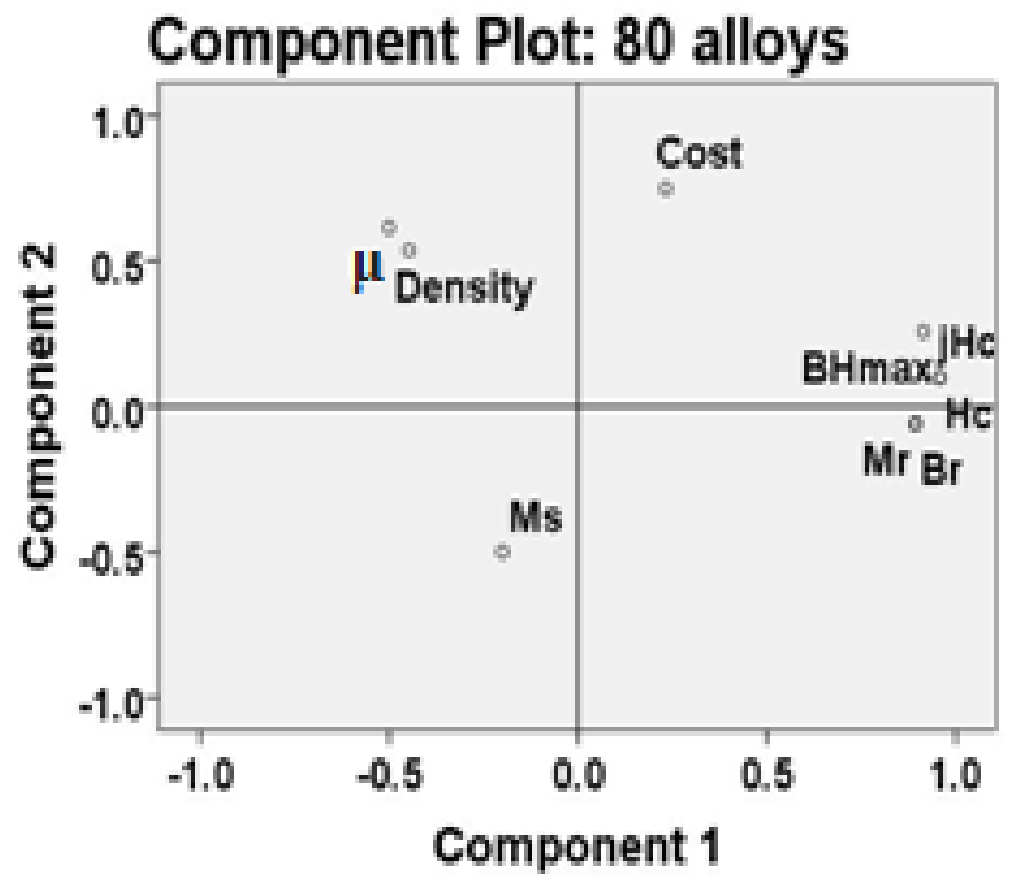

Figure 29: Orientation of various properties in the PC space

It can be seen that $H_{c}$ and $j H_{c}$ coincide at the same spot. It makes sense as one is the inverse of the other. Similarly, $M_{r}$ and $B_{r}$ can form a cluster and also $\mathrm{m}$ and density can be taken as another cluster. This means that properties that form a cluster are dependent on each other. Analysis of other data sets will further clarify these findings.

2. Optimization: Alloy $81-180$

With this data, we went for PC analysis of the elements. From scree plot in Figure 30 , it was found that 3 PC's were able to extract most of the information from the dataset. Figure 30 shows the scree plot for the elements while Figure 31 shows the position of various elements in the PC space. 


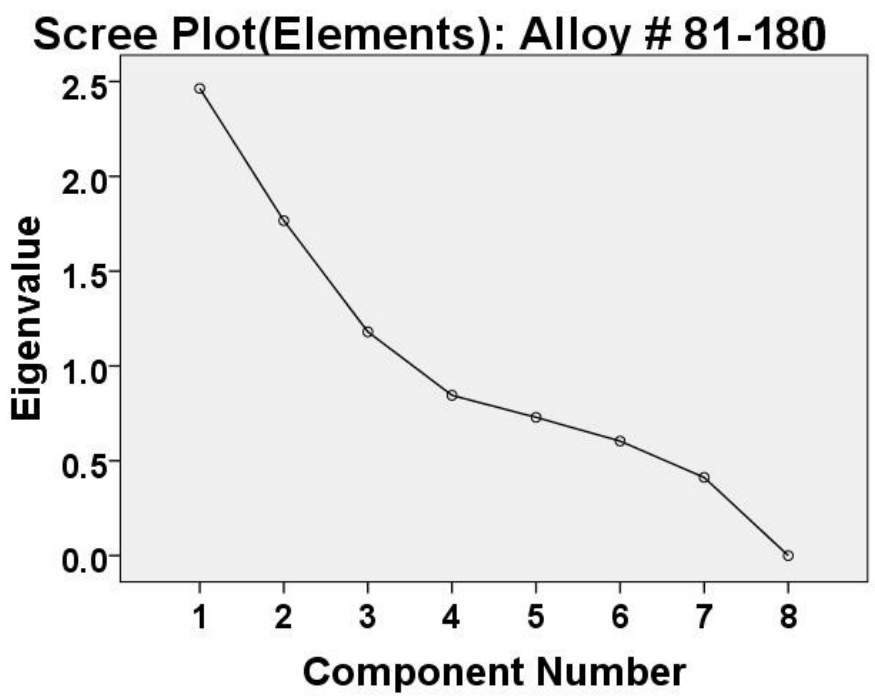

Figure 30: Scree plot for PCA analysis: 3 PCA components were chosen

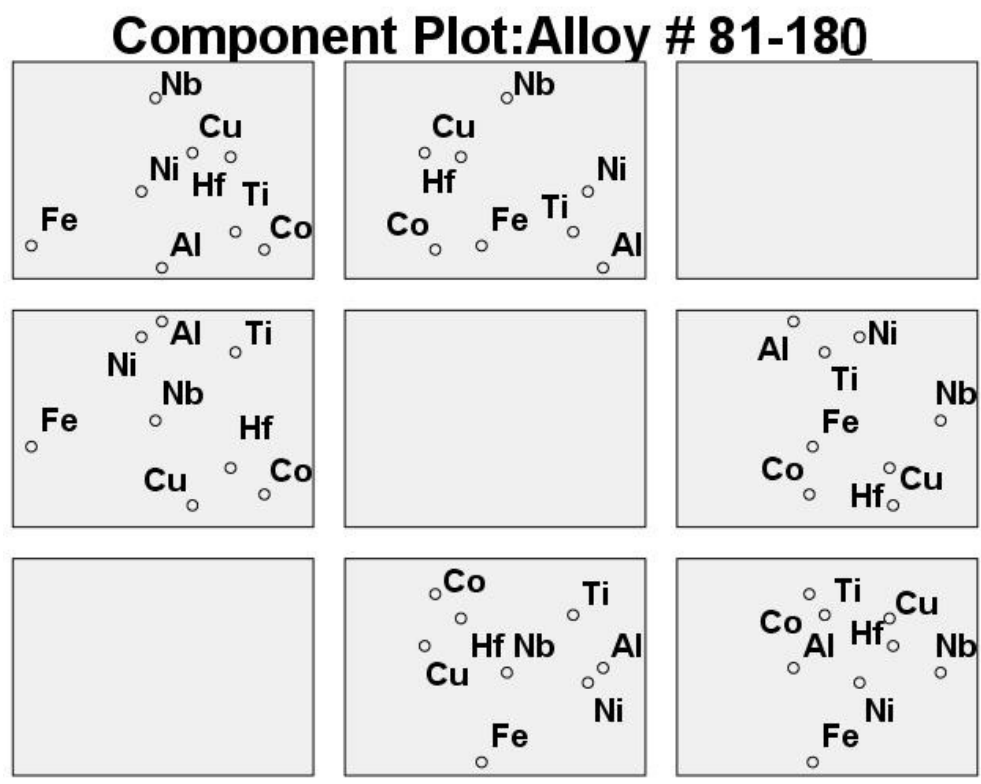

Figure 31: Orientation of various elements in the PC space

It can be observed that $\mathrm{Cu}$ and $\mathrm{Hf}$ seems to be part of a cluster. This means that there may exist $\mathrm{Cu}-\mathrm{Hf}$ rich precipitates in the alloy. Since, $\mathrm{Hf}$ precipitates at the grain boundaries. Also from SVR analysis, both $\mathrm{Cu}$ and $\mathrm{Hf}$ showed a direct response for $H_{c}$ and $B_{r}$. Hence, this must be analyzed further before moving for 
microstructure analysis. Additionally, $\mathrm{Ni}$ and $\mathrm{Al}$ too seem to be part of a cluster. This is quite evident in AlNiCo alloys as $\mathrm{Ni}-\mathrm{Al}$ rich phase $\left(\alpha_{2}\right)$ forms as a result of spinodal decomposition in AINiCo alloys.

From scree plot in Figure 32, it was found that 3 PC's were able to extract most of the information from the dataset.

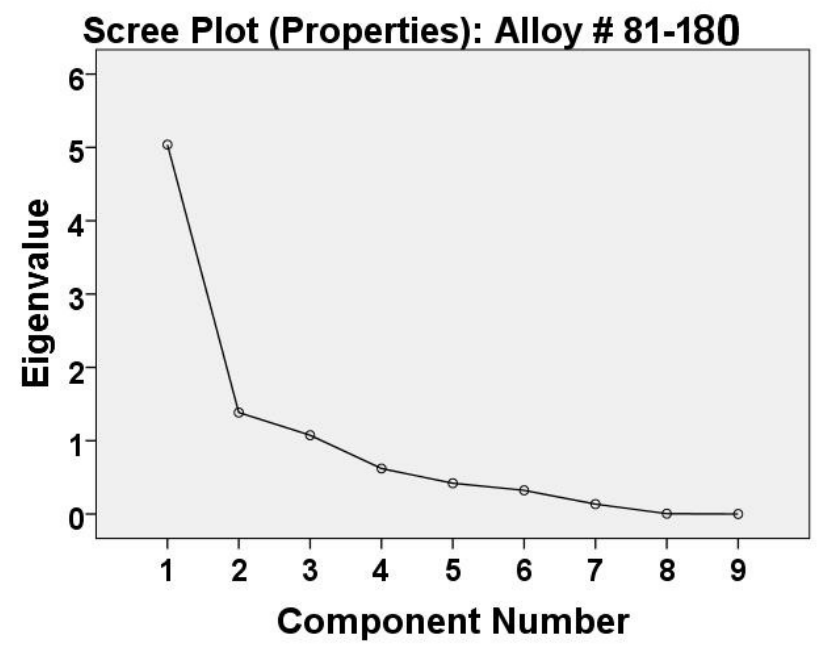

Figure 32: Scree plot for PCA analysis: 3 PCA components were chosen

Component Plot: Alloy \# 81-180

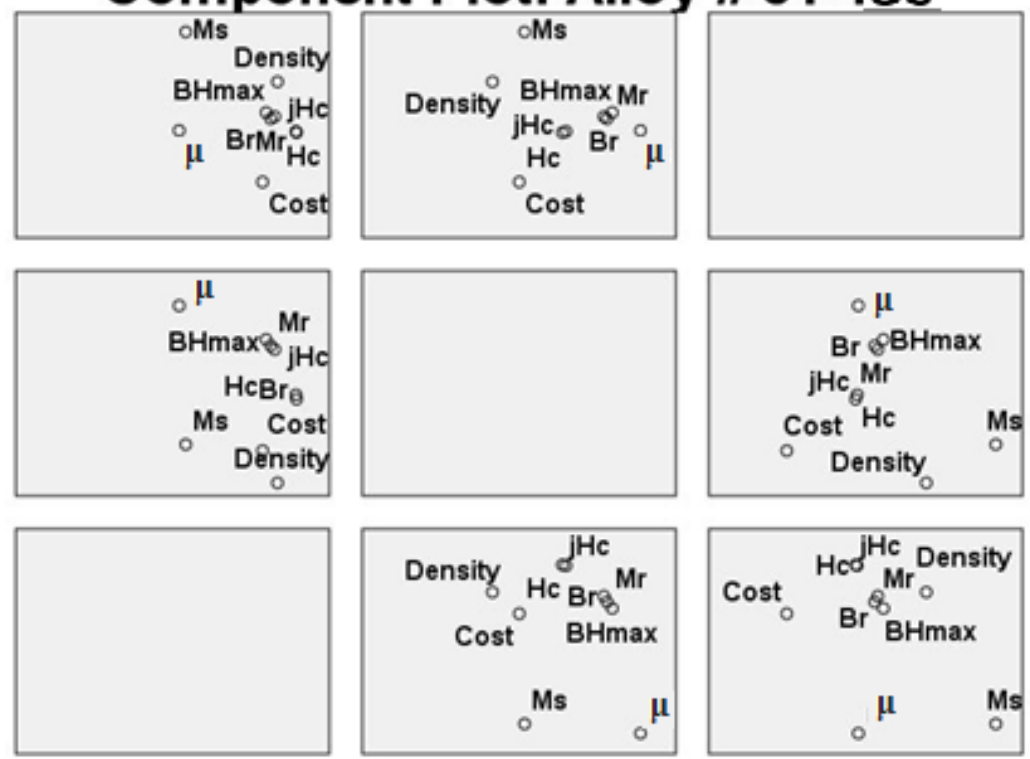

Figure 33: Orientation of various properties in the PC space 
Figure 32 shows the scree plot for the elements, while Figure 33 shows the position of various properties in the PC space. In Figure 33, $H_{c}$ and $j H_{c}$ are again coinciding. While, it can be seen that $B_{r}, M_{r}$ and $\left((B H)_{\max }\right)$ seems to be part of a cluster. Hence, these properties may be dependent on each other.

3. Data categorized on the basis of Multi-Criterion Decision Making (MCDM): 40 alloys were selected.

Due to software limitations, $(B H)_{\max }, H_{c}$ and $B_{r}$ were optimized while the other properties were predicted from meta-model. These properties equally important for the deployment of the magnet. In this part, 40 alloys were selected on the basis of objective defined in Table 2 by MCDM.

From Figure 34, one can observe that 3 PC's have eigenvalue greater that 1. So, it can be chosen for further analysis. Figure 35 shows the orientation of various elements in the PC space.

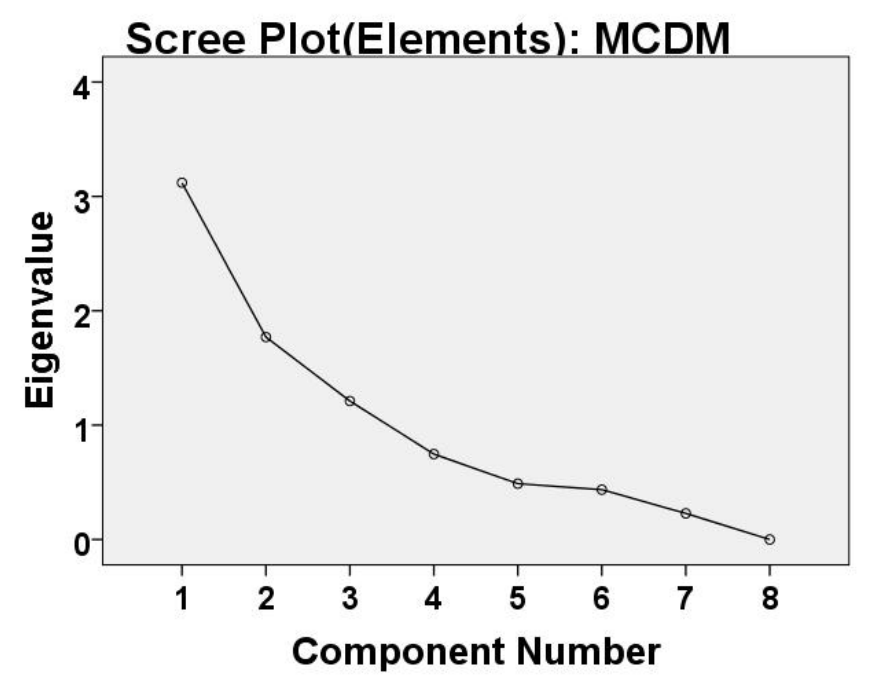

Figure 34: Scree plot for PCA analysis: 3 PCA components were chosen 


\section{Component Plot: MCDM}
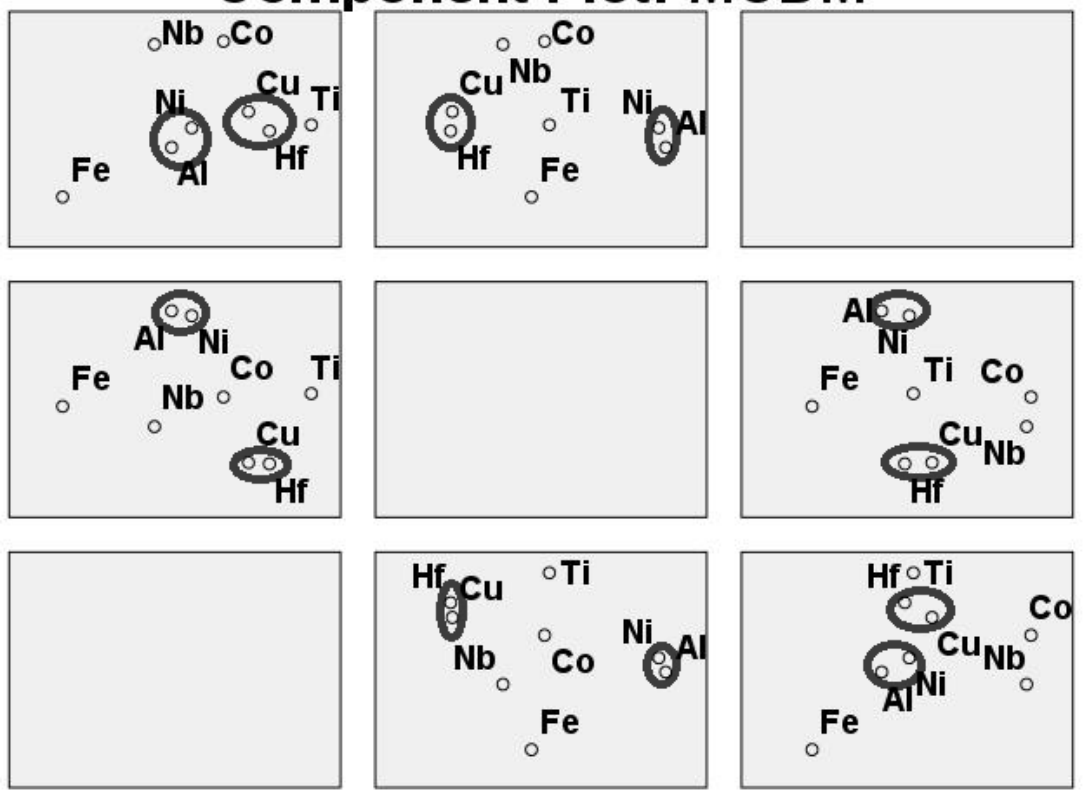

Figure 35: Figure 11: Orientation of various elements in the PC space

Figure 35 supports our finding that is an occurrence of $\mathrm{Cu}-\mathrm{Hf}$ cluster as well as $\mathrm{Ni}-\mathrm{Al}$ cluster. To further clarify it, we will proceed towards analyzing the whole data set.

Figure 35 shows a scree plot for various properties while Figure 37 shows the orientation of these properties in the PC space. 


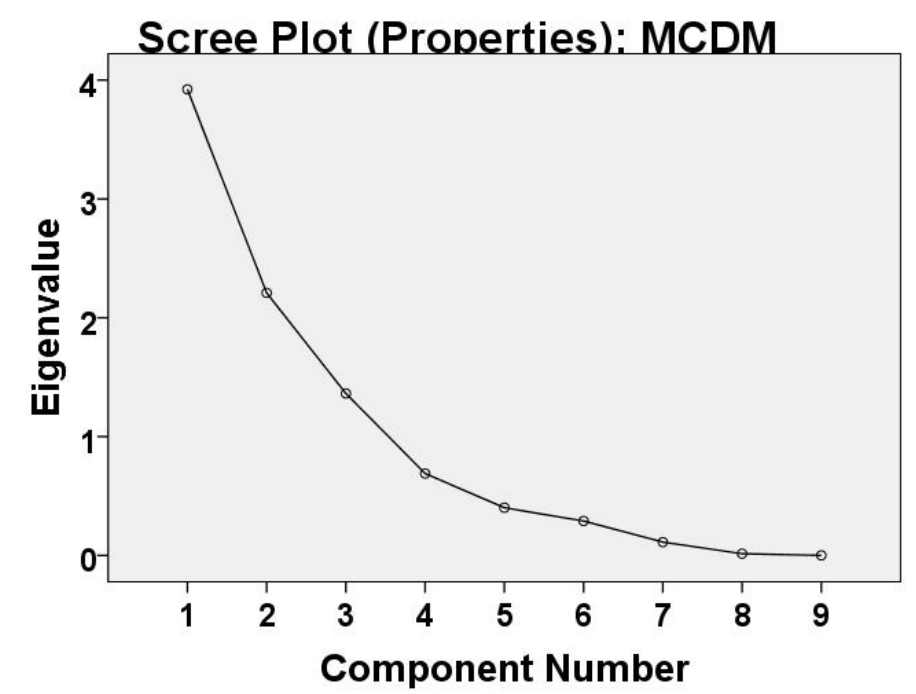

Figure 36: Scree plot for PCA analysis: 3 PCA components were chosen

Comnonent Plot: MCDM
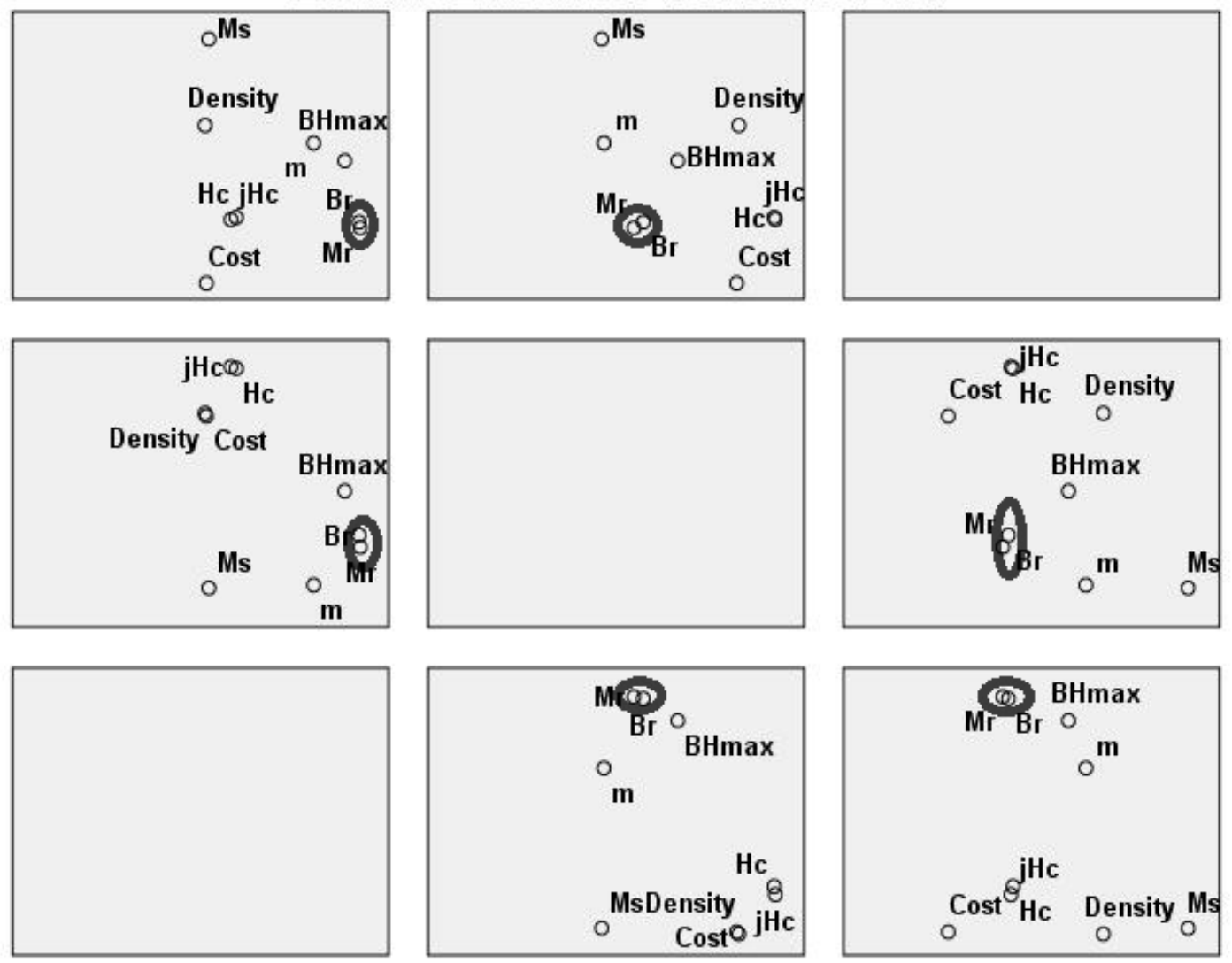

Figure 37: Orientation of various properties in the PC space 
$M_{r}$ and $B_{r}$ seems to form a cluster and hence these properties may be dependent on each other. $\left.(B H)_{\max }\right)$ does not seem to be part of the cluster anymore. Lastly, we can proceed towards analyzing the whole data set.

4. Whole dataset: Alloy 1-180. In this analysis, we used the complete dataset. Figure 38 shows the plot for various elements. It can be seen that 3 PC's are required to extract substantial information from the dataset. Figure 39 shows the orientation of various elements in the PC space.

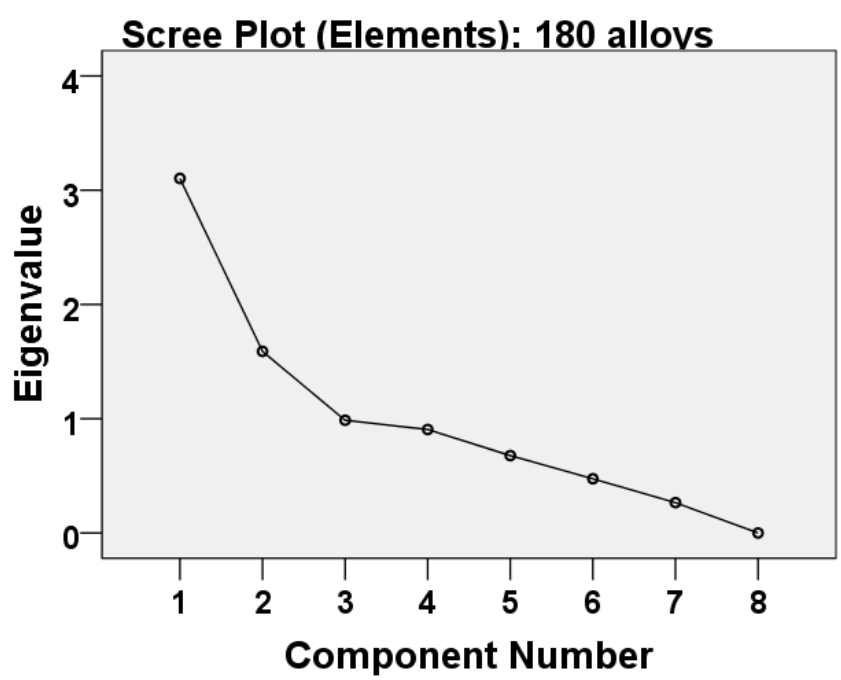

Figure 38: Scree plot for PCA analysis: 3 PCA components were chosen

In this set, we can see that $\mathrm{Cu}-\mathrm{Hf}$ seems to be part of the cluster. Here, in PC1 vs. PC2, we can see that Ti can also be considered to be part of this cluster. Nickel and Aluminum too forms a cluster. Hence, we have sufficient information from the above analysis to move forward towards microstructure analysis. 


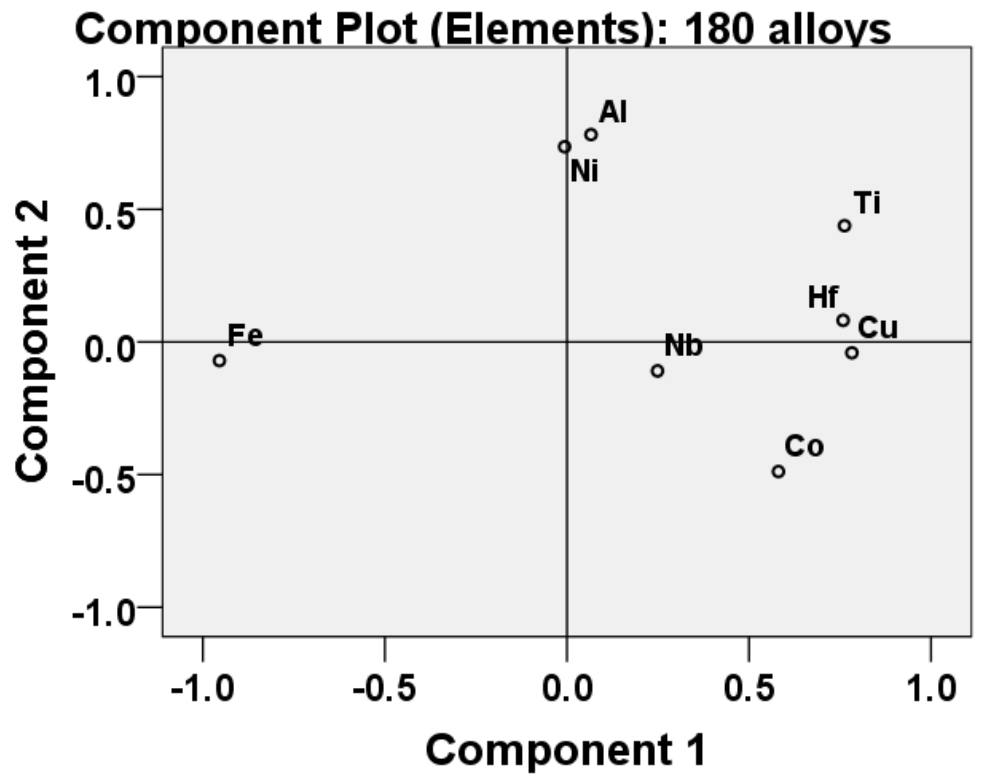

Figure 39: Orientation of various elements in the PC space

Figure 40 shows the scree plot for various properties while Figure 41 shows the orientation of various elements in the PC space.

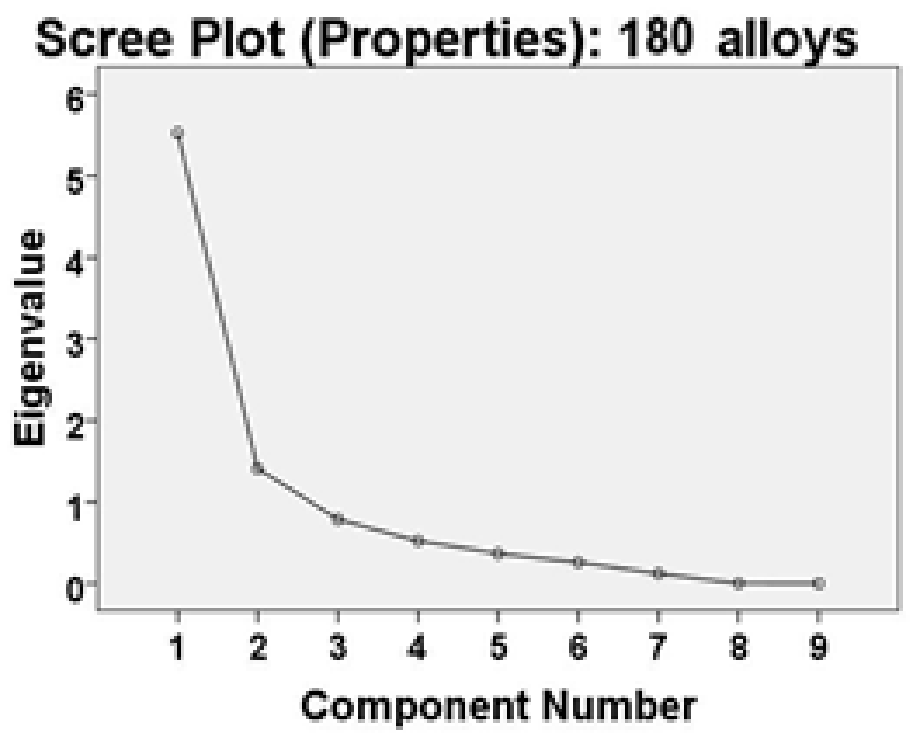

Figure 40: Scree plot for PCA analysis: 2 PCA components were chosen 
Here again form Figure 41, it seems that $(B H)_{\max }, B_{r}$, and $M_{r}$ forms a cluster.

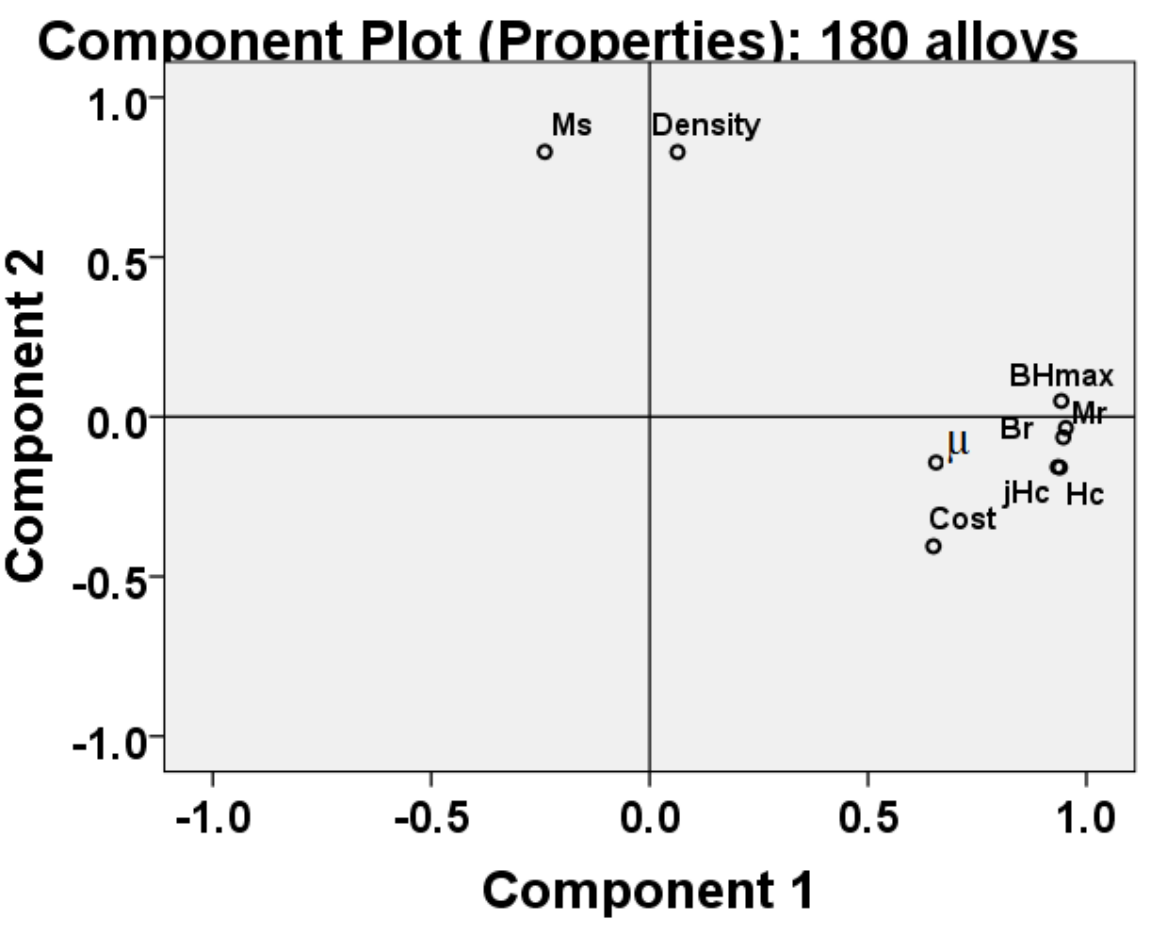

Figure 41: Orientation of various properties in the PC space

\subsubsection{PCA for materials discovery}

PC analysis has been successfully applied to materials discovery. Hence, one can test a new composition with the one available from a database to get any information regarding the property of interest. Hence, we did a cluster analysis on the PC of our data set. Here, we used the whole data set and marked the top 10 alloys on the basis of $(B H)_{\max }$ values. It can be seen from Figure 42, that these superior alloys cluster in a very small region while a majority of the PC space is covered by comparatively inferior alloys. Hence, if a certain composition is in the vicinity of these top 10 alloys, then they can be given a preference during the selection of alloys for experimental validation. 


\section{PCA (Elements): 180 alloys}

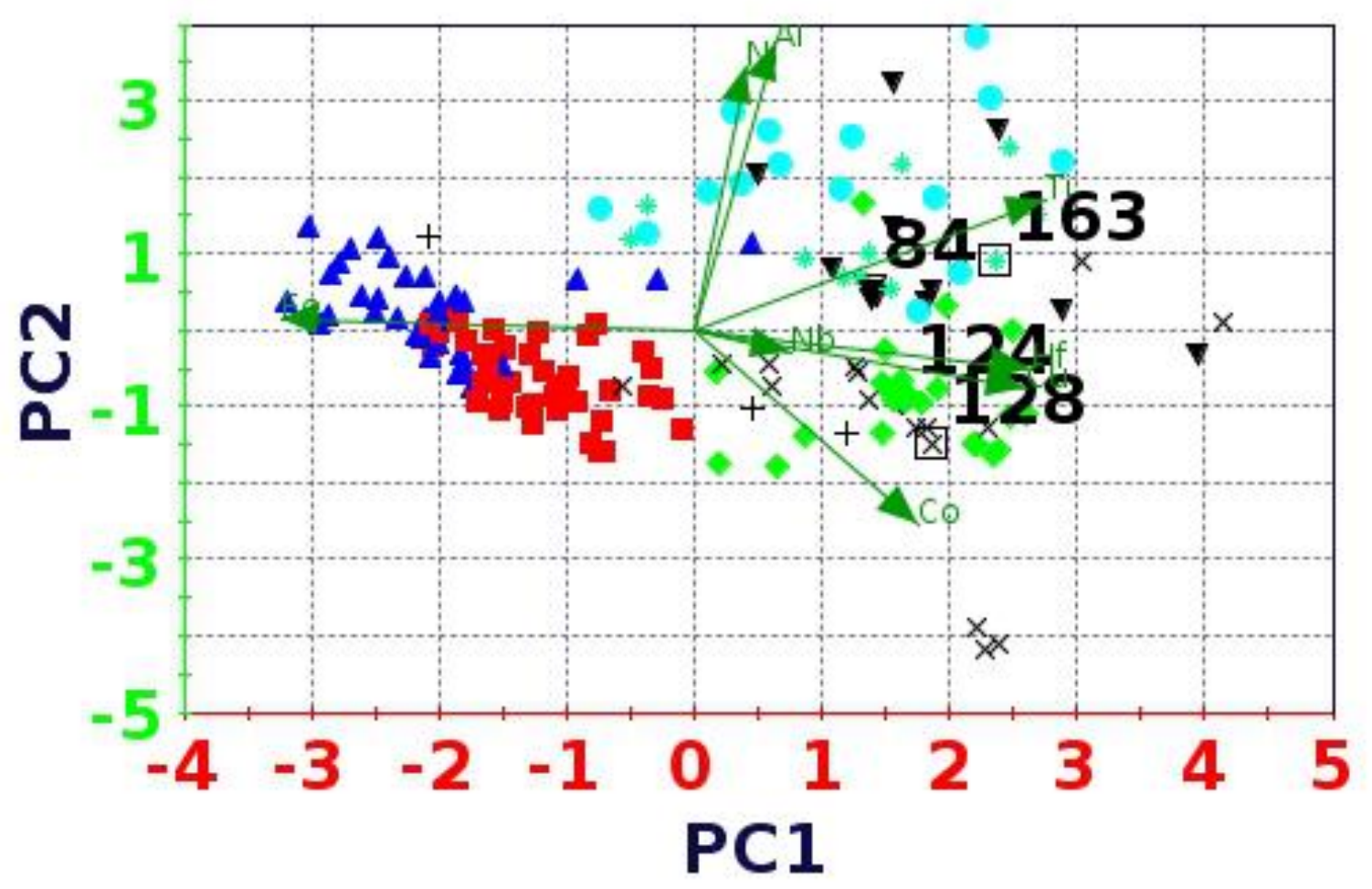

Figure 42: Orientation of various elements in the PC space

Figure 42 consists of all 180 alloys. Hence, it is a bit difficult to visualize. In this case, we used the dataset selected by MCDM and did the PC analysis of this dataset. Thereafter, we did a cluster analysis of the dataset. Figure 43 shows the orientation of various alloys in the PC space. 


\section{PCA (Elements): MCDM}

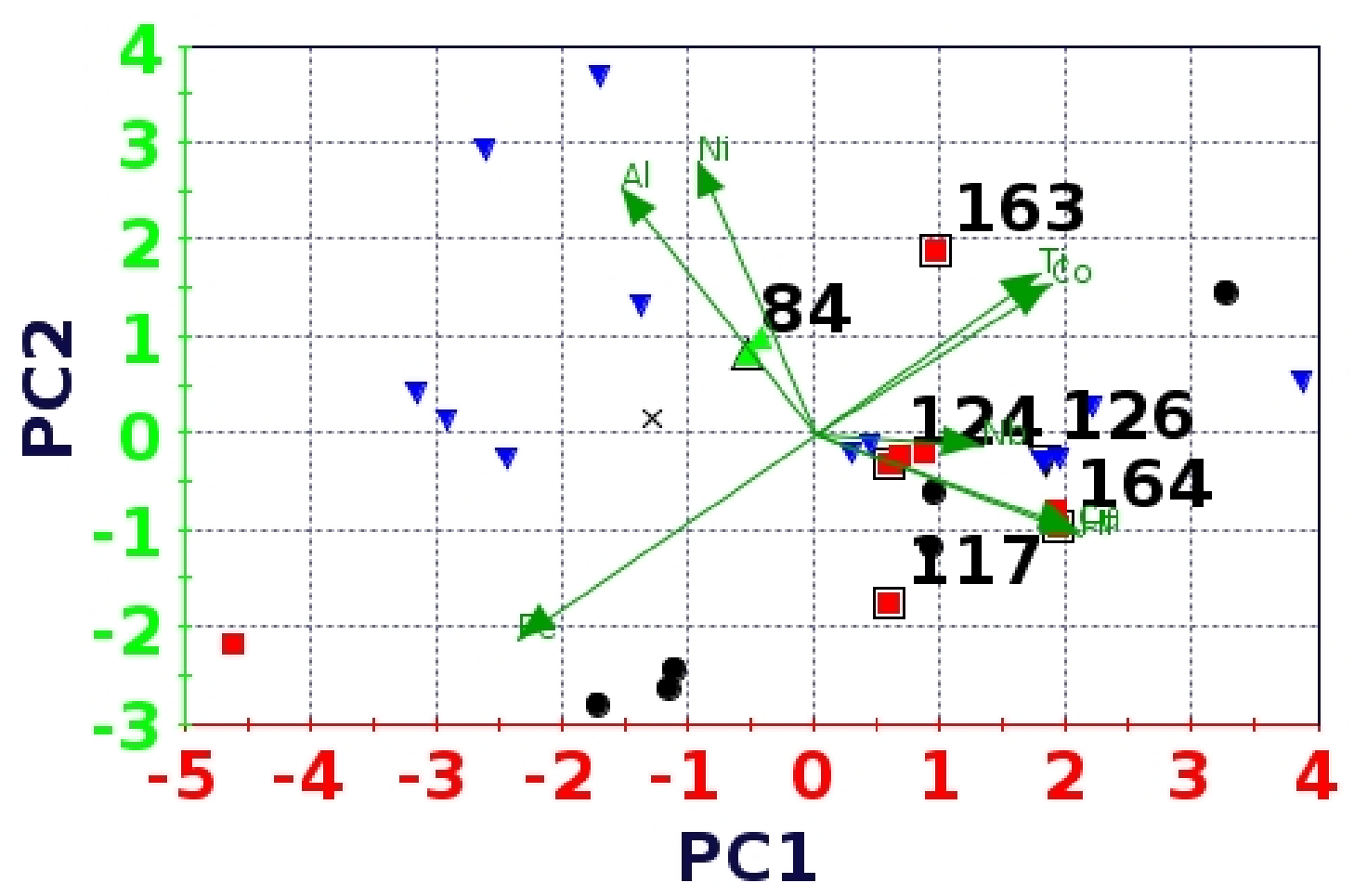

Figure 43: Orientation of various elements in the PC space

Top 10 alloys have been marked on Figure 42 and 43 . Since, it cannot be seen in the figure, alloys in the vicinity of these top 10 alloys were candidates that were part of the next set of best alloys. Hence, this method proves to be beneficial for the screening of the alloys prior to manufacture. 


\section{CHAPTER 7 DISCUSSIONS}

This chapter has been divided in two parts:

1. Data-driven

2. Experiments

\subsection{Data-driven approach}

In this part, we have summarized peculiar findings of our data-driven approach.

\subsubsection{Initial data-points}

Alloy development for AlNiCo alloys is a complex task as targeted properties are heavily dependent on thermo-magnetic protocol followed by a research group. During concentration variation for a particular thermo-magnetic protocol, usually concentration is varied for one or two elements while the rest of the elements, concentration remains fixed. This type of data can be used for regression models, but it will not be sufficient for development of meta-models due to lack of support points required for an accurate model. Additionally, it can be possible that the experimentalist may not have hit the optimum set of composition for a particular manufacture protocol.

In this work, we presented a novel approach to generate the initial dataset based on using an efficient random number generating algorithm, Sobols algorithm. These candidate alloys were checked for phase stability as a screening tool to come up with a set of composition which can be manufactured and tested for measuring bulk properties. 
Experimental modeling As mentioned in Chapter 3, experiments were conducted by our collaborator so we cannot discuss the process in this work. Here, we have listed the findings that have been presented at an international conference (Fan et al., 2016b) and published in a journal (Fan et al., 2016a) in section 7.2.

\subsubsection{Meta-modelling}

In this work, we used a set of approaches to develop meta-models for all the properties using different training and testing set. This was done in order to improve accuracy of the model for different sets of data. Thereafter, the model was screened on the basis of various accuracy measures described in Chapter 4 . Thereafter the models were tested for its ability to mimic information reported in the literature2. These findings were tabulated in Table 6. In SVR (Table 6), Nickel shows some weak response for $(B H)_{\max }$. Cu shows a direct correlation with $H_{c}$ and $B_{r}$ which can be confirmed from the literature (Section 2). Hf seems to affect $H_{c}$ and $B_{r}$ positively. Hence, initial study shows promising results. There is scope for improvement in the accuracy of response surface predictions.

The most accurate models screened on this basis have been tabulated in Table 5. These models have been used in prediction and multi-objective optimization of targeted properties. A similar approach is quite popular in ensemble learning (parallel and distributed data mining) for selection of the classification algorithm.

\subsubsection{Multi-objective optimization}

Due to software limitations, three properties were optimized at a time. 
Multi-objective optimization of targeted properties were performed by using a set of evolutionary algorithms (as discussed in Chapter 3 and Chapter 4) to explore the search space efficiently. Remaining seven properties were predicted from the chemical composition of the new Pareto-optimized candidate alloys by using the meta-models developed in the previous step.

This data was used by our collaborators, Professor Egorov, Professor Colaço and Professor Choudhury, where they used their algorithms to develop meta-models that are conceptually different from that used in our lab. Our collaborators provided us with their Pareto-optimized predictions after each cycle. Hence, we ended up with a large set of Pareto-optimized predictions from which we needed to choose a few candidates for manufacture and testing in the next cycle.

\section{MCDM}

Dataset obtained after multi-objective optimization (section 7.1.3) was further screened using MCDM approach. Based on algorithms prediction and our knowledge, a set of candidate alloys were selected for further analysis. This dataset was further screened by PCA and HCA analysis. During PCA and HCA analysis, experimentally verified alloys were added to the Pareto-optimized dataset. In PCA and HCA analysis, preference was given to the alloys that were clustered with best alloys over the cycles. On the basis of MCDM, PCA and HCA, a set of candidate alloys were selected in each cycle (tabulated in Table 2) for the manufacture and testing of macroscopic properties. 


\subsubsection{Unsupervised learning approach}

\section{PCA}

PC analysis proved to be helpful in reducing the dimensionality of the data set for visualization. PC analysis points towards a correlation between elements $\mathrm{Cu}-\mathrm{Hf}$ and $\mathrm{Ni}-\mathrm{Al}$. Ni-Al rich phase is known in AINiCo alloys and its effect on magnetic properties is supported by data from the literature. Hf has been rarely used in AINiCo alloys and hence its similarity with $\mathrm{Cu}$ can be exploited to improve the magnetic properties. Hf enhances high temperature properties, hence the new magnets are supposed to have superior magnetic properties at elevated temperatures.

From Figures 42 and 43 , one can see that $\mathrm{Nb}$ has the lowest contribution on PC1, although it is collinear to it. Niobium is almost orthogonal to PC2 and hence, it will have the least contribution to it. This suggests that if one needs to exclude an element from further analysis, one can think of excluding $\mathrm{Nb}$ and manufacture and test a few samples without it.

These findings are quite helpful in the development of a knowledge base for the design of new materials. At the same time, it has the potential to save time and money otherwise invested in random experimentation. PC analysis can be used as a tool to screen alloys predicted by various optimizers prior to manufacture. Alloys that are near to the previous best alloys in the PC space can be preferred to manufacture over the others for improved results.

At present, ab-initio based calculations, as well as Calphad approach, are effective for limited systems (alloys having maximum 3-4 elements), and cannot 
handle eight elements. Use of statistical tools will be helpful in determining the most influential alloying elements. This will be helpful in theoretical validation of the above findings. Additionally, one can work on finding the most stable phases needed for enhanced performance of these alloys by focusing on the most influential elements.

\section{HCA}

In this work, we demonstrated on how HCA analysis can be used to screen alloys prior to development in the future. $H_{c}$ increases with increase in copper content. This was observed in the SVR analysis (Section 5.2.1), as well as from the literature (Section 2). Nickel shows mixed response for $(B H)_{\max }$ as observed in the SVR analysis (Section 5.2.1). Titanium showed a mixed response for $H_{c}$. From the above analysis, we can conclude that we can remove Niobium in order to make way for a rare - earth addition.

On the basis of limited knowledge of the literature and mixed SVR analysis results, we were able to predict the composition range of quite a few elements for optimized properties by HCA analysis.

\section{Thermodynamic analysis}

Thermodynamic analysis can prove to be helpful in designing heat treatment protocol. Equilibrium calculations can be used to screen a few alloys prior to manufacture.

At present, we are using the 8 elements. It will be helpful for an experimentalist to have an idea regarding the stability of critical phases during 
manufacture/ designing thermo-magnetic treatment protocol. In this work, we studied phase stability of a few alloys from $0{ }^{\circ} \mathrm{C}$ to $1200{ }^{\circ} \mathrm{C}$ in Factsage. These diagrams can act as a guideline for the experimentalist while selecting alloys prior to manufacture (Jha et al., 2016).

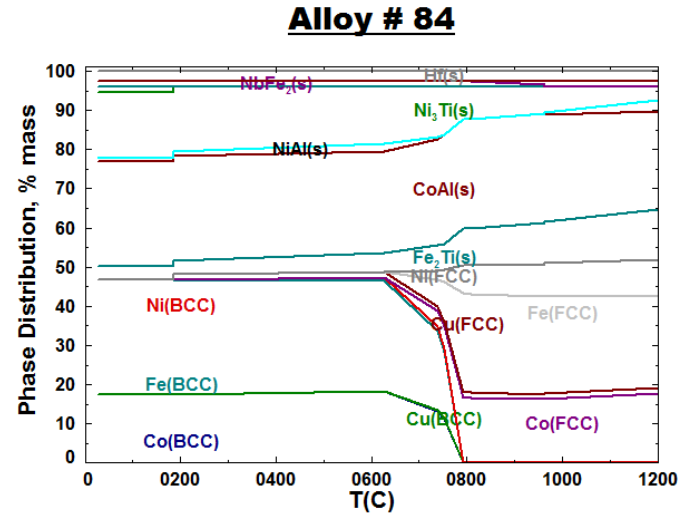

(a)

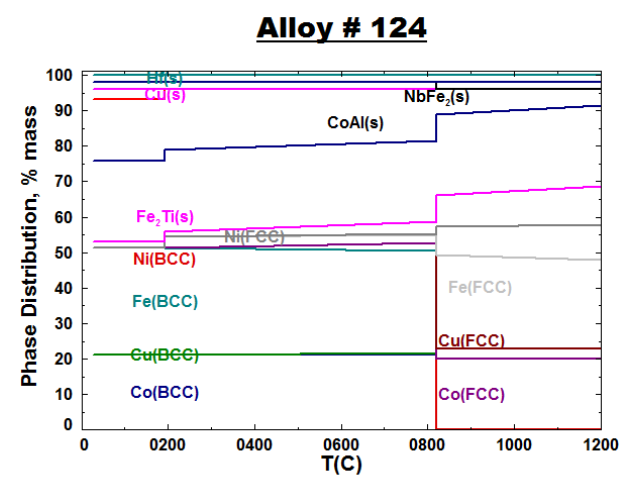

(c)
Alloy \# 86

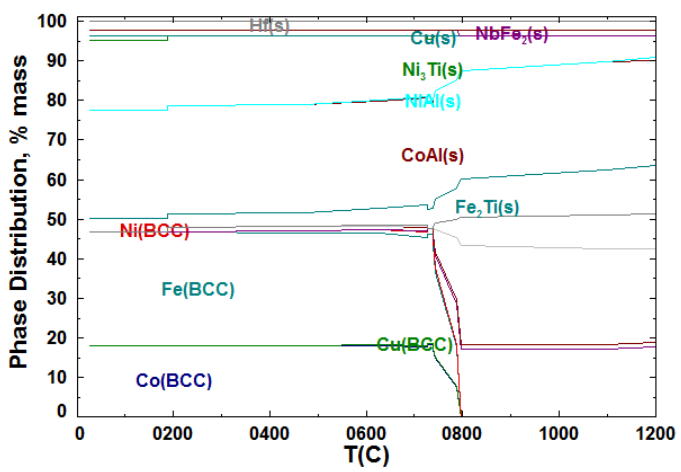

(b)

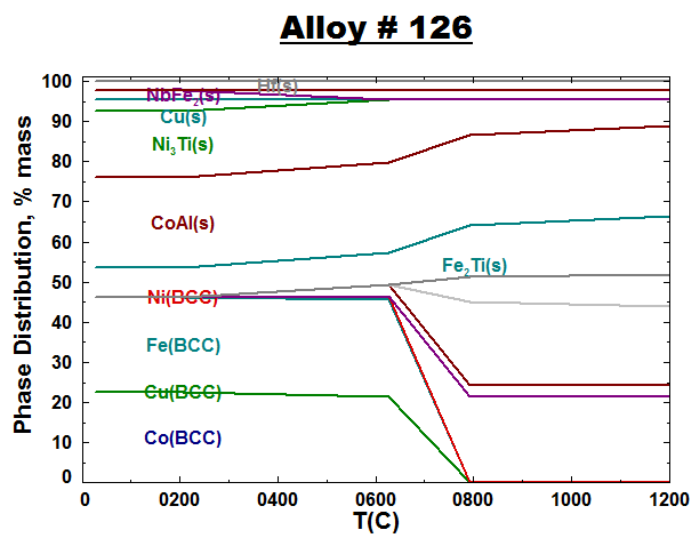

(d)

Figure 44: Phase distribution diagrams for alloys: (a) 84, (b) 86, (c) 124 and (d) 126

From Figure 44, it can be observed that alloy 124 is thermodynamically stable up-to $800^{\circ} \mathrm{C}$. While in alloys 84,86 and 126 , transformation (BCC-FCC) starts at lower temperatures. Hence, an experimentalist can design a heat 
treatment protocol, so that he can avoid transformations that will have a detrimental effect on the magnetic properties.

We extended this analysis by modifying the composition of Alloy 124 . We added Mn in various amounts and plotted the critical phases.

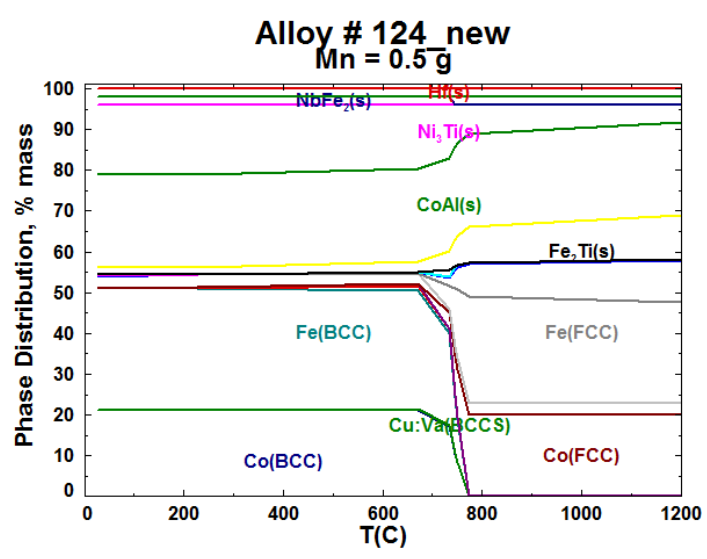

[ $0.5 \mathrm{gm} \mathrm{Mn}$ added to alloy 124$]$

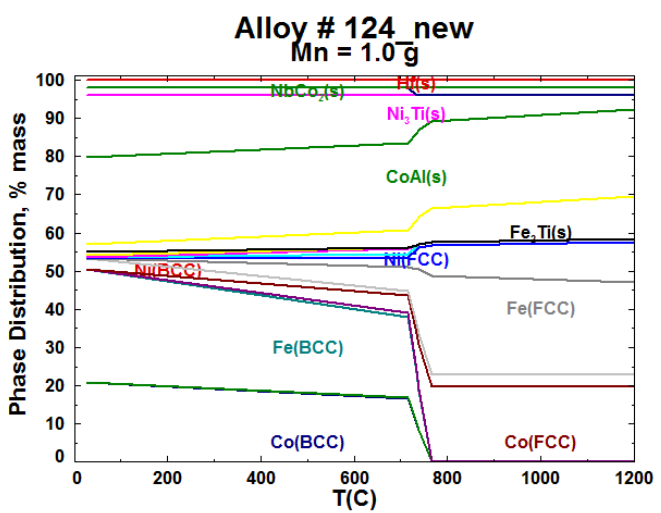

[1.0 gm Mn added to alloy 124]

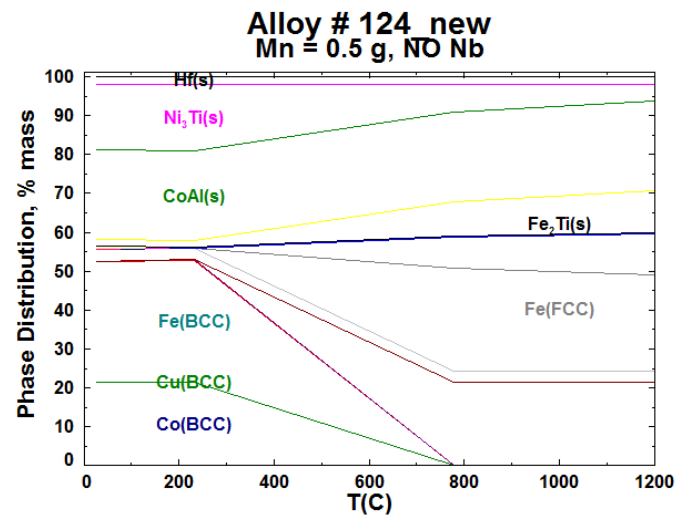

[0.5 gm Mn added to alloy 124 and $\mathrm{Nb}$ removed]

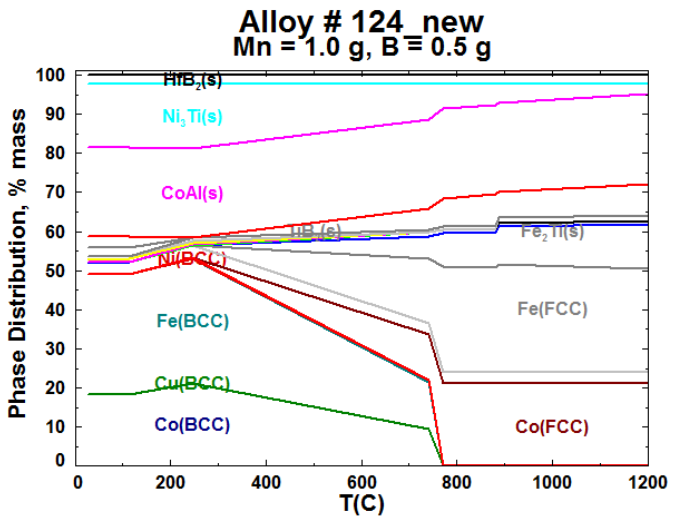

[1.0 gm Mn and $0.5 \mathrm{~g} \mathrm{~B}$ added to alloy 124]

Figure 45: Phase distribution diagram obtained after modifying the composition of Alloy \#124 
From Figure 45, it can be seen that these additions had detrimental effect and BCC-FCC transformation started well below $800^{\circ} \mathrm{C}$. Hence, at this point we can say that we must not go for Mn and B addition.

\subsection{Experiments}

Experiments were carried out at North Carolina State University and our collaborators focussed on a few peculiar aspects of AINiCo alloys at nano-scale level, which has been pointed out in literature but a thorough investigation was not possible due to lack of characterization tools.

Experimental procedure can be summarized as follows:

1. Development of a standardized thermo-magnetic protocol to be followed for the initial set of alloys.

2. Optimizing thermo-magnetic protocol by adding a tempering step besides the standardized process. Thermo-magnetic protocol is part of the technical report submitted to AFOSR and hence it is not reported in this work.

3. A thorough research on effect of titanium on the formation and evolution of $\mathrm{Cu}-\mathrm{Ni}$ rich bridge between adjacent $\alpha_{1}$ phase. This work has been presented at an international conference (Fan et al., 2016b) and has been accepted for publication (Fan et al., 2016a). In this work, we have explained peculiar findings from this work.

4. A thorough research on effect of tempering on the formation and evolution of Cu-Ni rich bridge between adjacent $\alpha_{1}$ phase (Fan et al., 2016c). This work is not published, hence it will not be reported in this work (Fan et al., 2016a).

7.2.1 Characterization of alloy \# 95 (Fan et al., 2016a; 2016b) 
Alloy \#95 was checked for compositional homogeneity by optical microscopy, and EDS analysis.

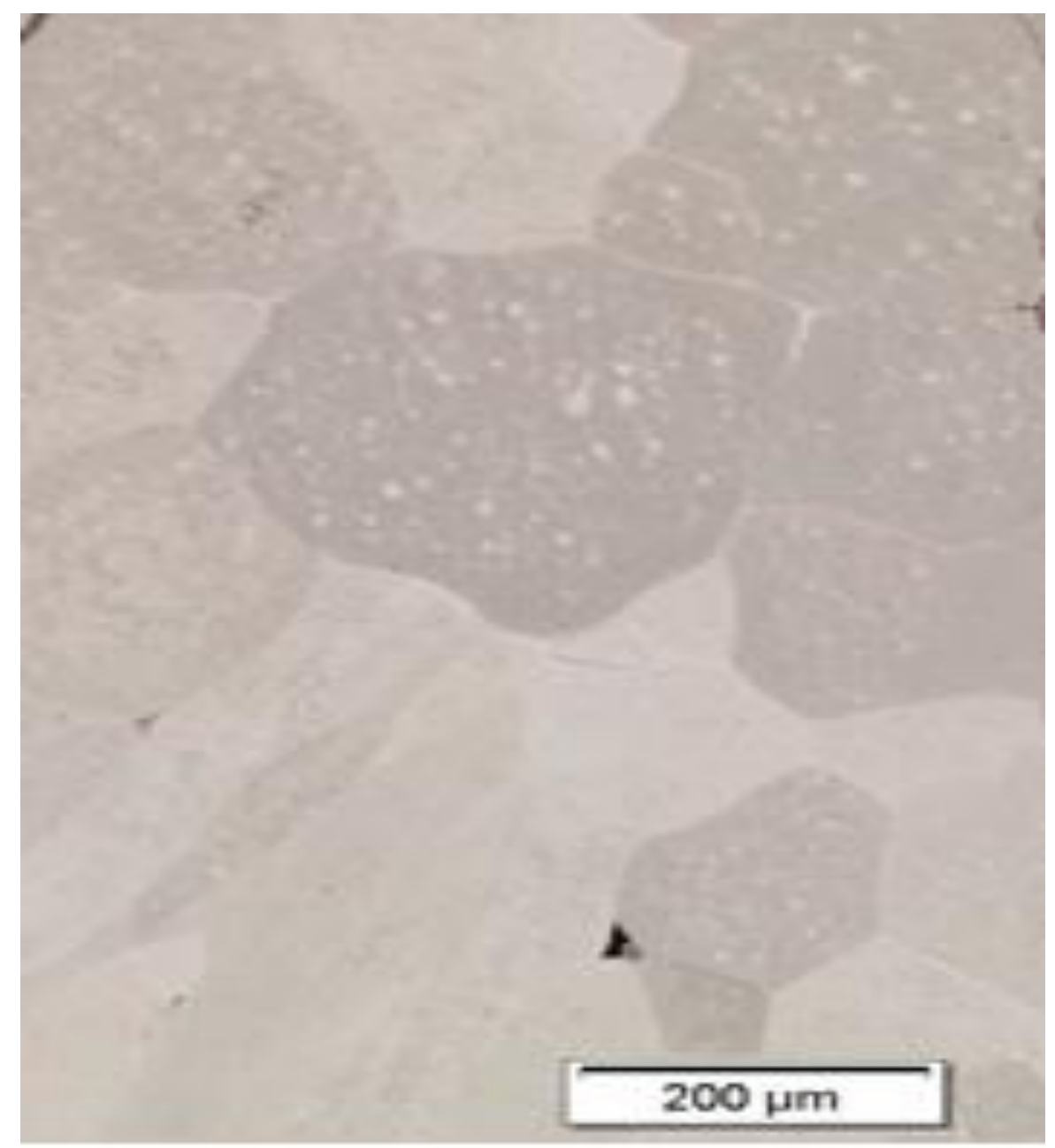

Figure 46: Optical micrograph for alloy \# 95 showing white spots

Several white precipitates were observed in the optical micrograph as can be seen in Figure 46. A recent work (Xing et al., 2013) has mentioned about appearance of white spots in AINiCo 8 and 9 due to the formation of copper and titanium precipitates. As mentioned in Chapter 2, both $\mathrm{Ti}$ and $\mathrm{Cu}$ forms that are helpful in refining the alloy from impurities. It was also also mentioned about the 
role of copper precipitates in separation of $\alpha_{1}$ phases that proved to be helpful in improving $H_{c}$ and $B_{r}$ of the alloys (Xing et al., 2013) .

Thereafter, the sample was analyzed by SEM where backscattered image was used for compositional mapping as shown in Figure 47 and the composition has been tabulated in Table 8 (Fan et al., 2016a; 2016b).

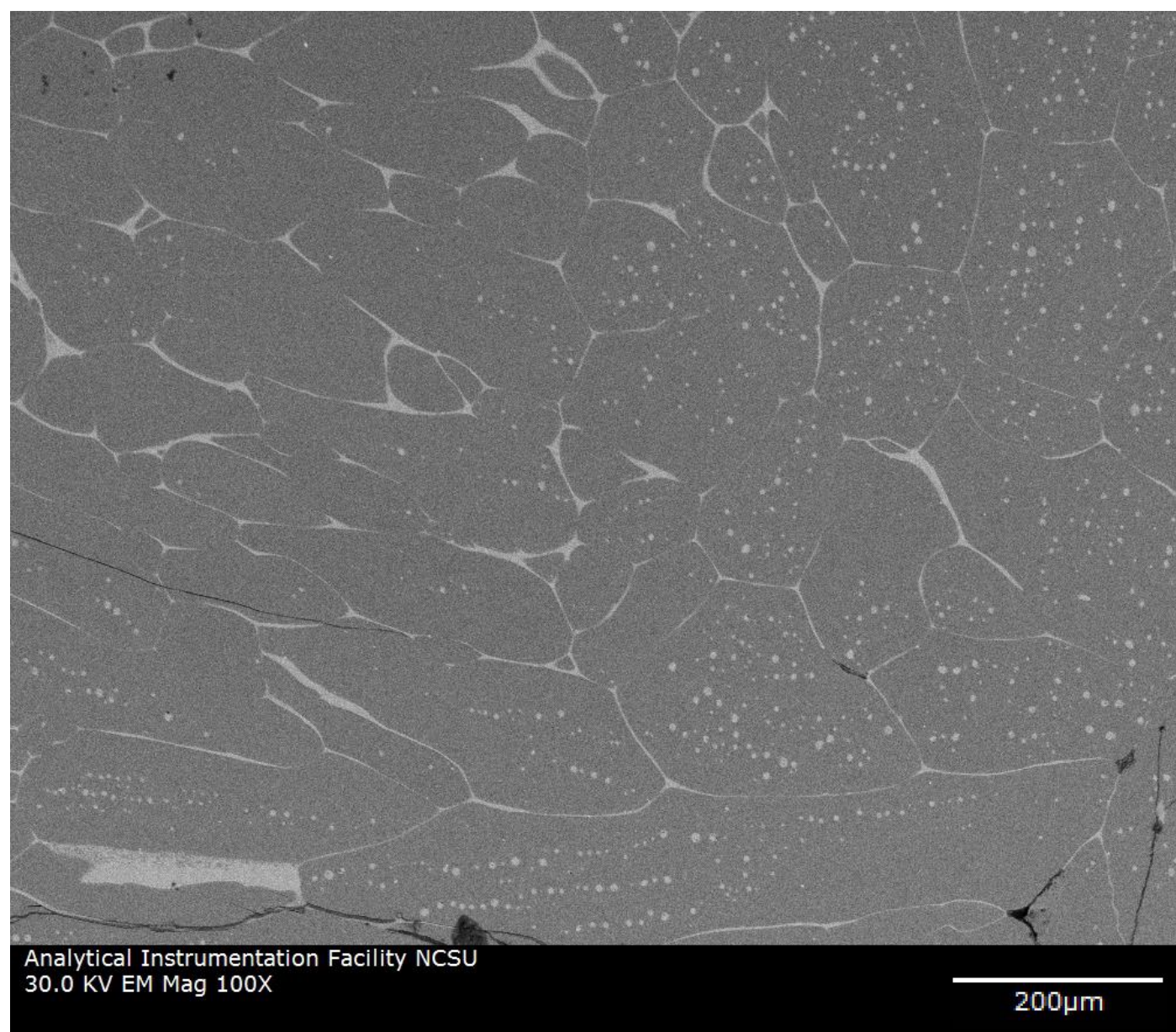

Figure 47: Back scattered image used for compositional mapping of alloy \# 95 viewings along the transverse orientation (parallel to the magnetic field) 
Nominal composition is almost same as the composition of the whole image as can be observed from Table 8. Thereafter, an EDS analysis was performed by scanning at specific points marked in Figure 48 (Fan et al., 2016a; 2016b).

Table 8: Composition mapping of BSE image for alloy \#95

\begin{tabular}{|c|c|c|}
\hline & Nominal composition & Whole image \\
\hline $\mathrm{Fe}$ & 32.3595 & 32.09 \\
\hline $\mathrm{Co}$ & 36.8574 & 35.64 \\
\hline $\mathrm{Ni}$ & 13.5449 & 11.99 \\
\hline $\mathrm{Al}$ & 7.2002 & 8.76 \\
\hline $\mathrm{Ti}$ & 4.1162 & 5.21 \\
\hline $\mathrm{Hf}$ & 2.0683 & 0.04 \\
\hline $\mathrm{Cu}$ & 2.9385 & 2.56 \\
\hline $\mathrm{Nb}$ & 0.9307 & 1.32 \\
\hline $\mathrm{C}$ & 0 & 1.54 \\
\hline $\mathrm{O}$ & 0 & 0.86 \\
\hline
\end{tabular}

EDS analysis points towards iron deficient region at the grain boundaries and white precipitates. There is no trace of $\mathrm{Hf}$ in the matrix and all of $\mathrm{Hf}$ is precipitated at the grain boundary and in the white precipitates. Concentration of $\mathrm{Cu}$ and $\mathrm{Ti}$ is comparatively higher on GB and white spot when compared to nominal composition (Table 9) (Fan et al., 2016a; 2016b). 
Magnetic properties of AINiCo magnets depend on shape anisotropy and spinodal refinement at nano-scale level. Hence, the research team at NCSU, took a complex task of characterizing two samples from micro-scale to nano-scale and finally to the atomic scale.

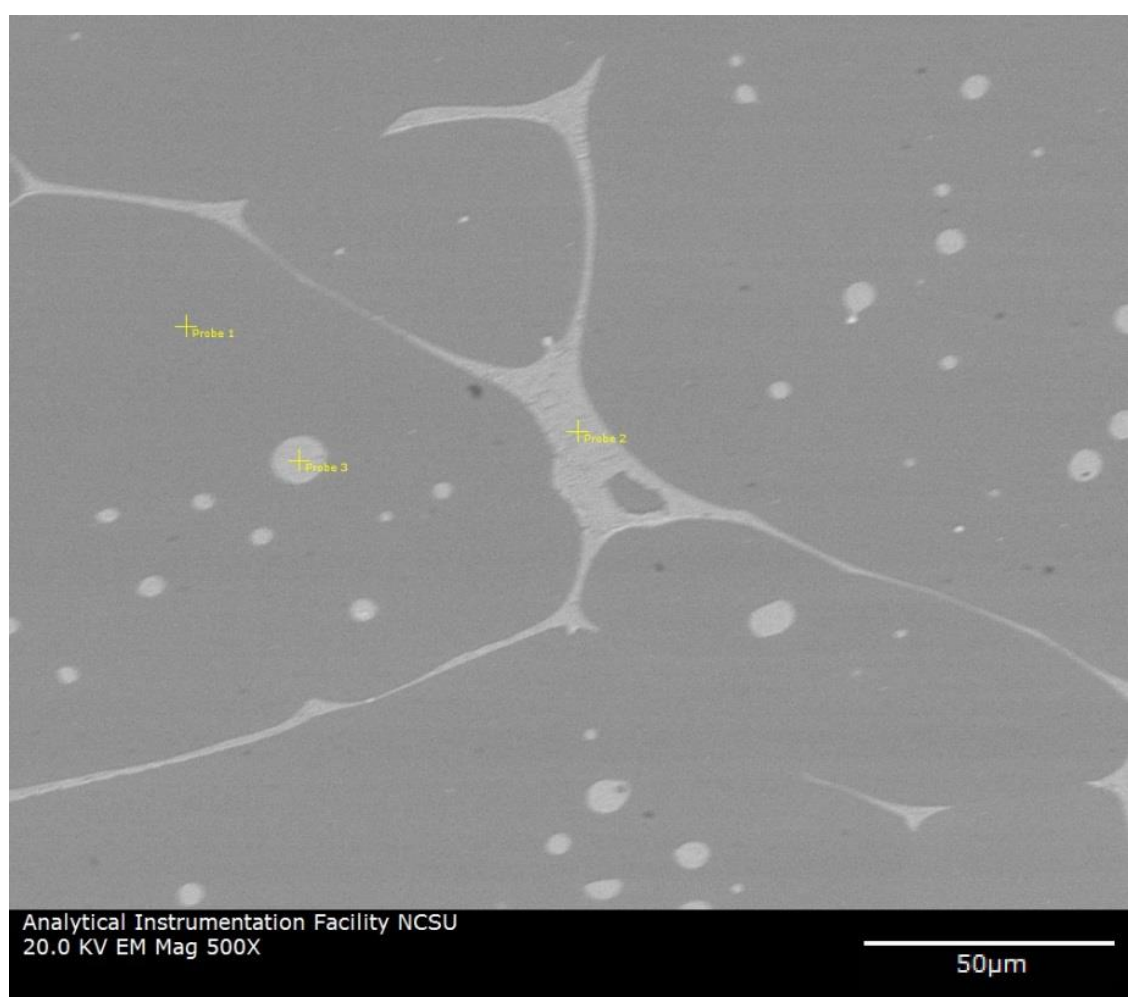

Figure 48: SEM image showing the white spots to be be analyzed by EDS for alloy \# 95 viewings along the transverse orientation (parallel to the magnetic field) 
Table 9: EDS analysis of white spots observed in SEM micrograph for alloy \#95

\begin{tabular}{|l|r|r|r|r|}
\hline Element & $\begin{array}{r}\text { Nominal } \\
\text { composition }\end{array}$ & \multicolumn{1}{c|}{$\begin{array}{c}\text { Point 1 } \\
\text { (Grain) }\end{array}$} & $\begin{array}{r}\text { Point 2 } \\
\text { (Grain boundary) }\end{array}$ & $\begin{array}{r}\text { Point 3 } \\
\text { (White spots) }\end{array}$ \\
\hline $\mathrm{Fe}$ & 32.3595 & 32.50 & 23.88 & 25.78 \\
\hline $\mathrm{Co}$ & 36.8574 & 35.45 & 34.79 & 34.49 \\
\hline $\mathrm{Ni}$ & 13.5449 & 14.32 & 14.99 & 14.43 \\
\hline $\mathrm{Al}$ & 7.2002 & 9.67 & 6.85 & 8.88 \\
\hline $\mathrm{Ti}$ & 4.1162 & 4.30 & 4.78 & 4.92 \\
\hline $\mathrm{Hf}$ & 2.0683 & 0 & 7.95 & 5.89 \\
\hline $\mathrm{Cu}$ & 2.9385 & 3.32 & 3.77 & 3.42 \\
\hline $\mathrm{Nb}$ & 0.9307 & 0.44 & 2.96 & 2.17 \\
\hline $\mathrm{C}$ & 0 & 0 & 0.03 & 0.03 \\
\hline $\mathrm{O}$ & 0 & 0 & 0 & 0 \\
\hline
\end{tabular}

\subsubsection{Characterization of $\mathrm{Cu}-\mathrm{Ni}$ rich bridges}

The aim of this work was further divided into two parts:

1. Evolution and formation of $\mathrm{Cu}-\mathrm{Ni}$ rich bridges during spinodal decomposition.

2. Study the effect of tempering on the growth of Cu-Ni rich bridges.

In this work, we have discussed upon the evolution and formation of $\mathrm{Cu}-\mathrm{Ni}$ rich bridges during spinodal decomposition. Hence, the magnetic properties were not optimized in this work. 


\section{Evolution and formation of $\mathrm{Cu}-\mathrm{Ni}$ rich bridges during spinodal}

\section{decomposition}

The various steps involved in this work can be listed as follows:

\section{Sample preparation}

Two samples were prepared with chemical composition as listed in Table 10. It can be observed that Sample A contains Ti, while Sample B does not contain Ti and an equivalent weight was added to the Fe content in Sample B. Chemical concentration of the rest of the elements remains unaltered.

This chemical composition serves two purposes:

1. Regarding chemical composition, Sample A resembles AINiCo 9, while sample B resembles AINiCo 5. This will help in comparing the properties of these alloys with the commercial alloys. Such a comparison will be beneficial for the reader in understanding our motive even though the commercial AlNiCo alloys are exposed to different thermo-magnetic protocol.

2. It will provide information on the effect of $\mathrm{Ti}$ on the evolution of $\mathrm{Cu}$ Ni bridge.

Table 10: Chemical composition of samples A and B

\begin{tabular}{|c|c|c|c|c|c|c|c|c|}
\hline Sample & \multicolumn{7}{|c|}{ Composition (Wt. \%) } \\
\hline & Fe & Co & $\mathrm{Ni}$ & $\mathrm{Al}$ & $\mathrm{Ti}$ & $\mathrm{Hf}$ & $\mathrm{Cu}$ & $\mathrm{Nb}$ \\
\hline $\mathrm{A}$ & 32.3 & 36.9 & 13.5 & 7.2 & 4.1 & 3.0 & 2.1 & 0.9 \\
\hline B & 36.5 & 36.9 & 13.5 & 7.2 & 0 & 3.0 & 2.1 & 0.9 \\
\hline
\end{tabular}




\section{Microstructure}

Figure 49 corresponds to HAADF image with EDS mapping for Sample A with $\mathrm{Ti}$, while Figure 50 corresponds to EDS mapping for Sample B without Ti. For sample A (Figure 49), Fe-Co rich hard magnetic $\alpha_{1}$ phase can be clearly distinguished from the dark matrix of $\mathrm{Ni}-\mathrm{Al}$ rich soft magnetic $\alpha_{2}$ phase. Additionally, there exists Cu-rich areas in the form of small and bright circles which appears to bridge adjacent $\alpha_{1}$ phases. For sample B (Figure 49), $\alpha_{1}$ does not process well defined boundaries and seems to be overlapping with $\alpha_{2}$ phases. Additionally, Cu-Ni rich bridge is absent in Figure48 and $\mathrm{Cu}$ is dispersed in $\alpha_{2}$ phases. This shows that the addition of Ti in Sample A was the driving force behind significant change in morphology after spinodal decomposition. 

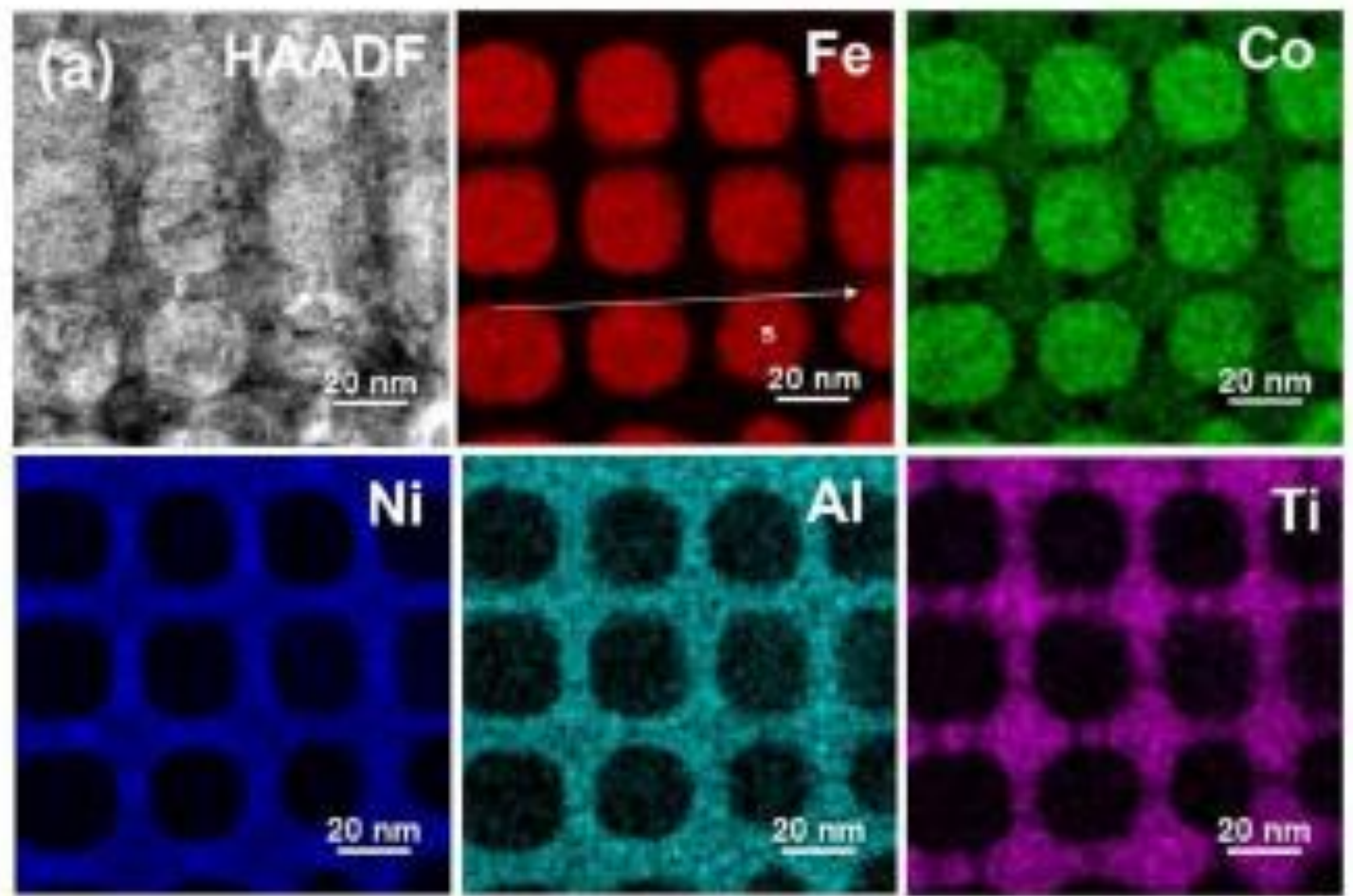

$20 \mathrm{~nm}$
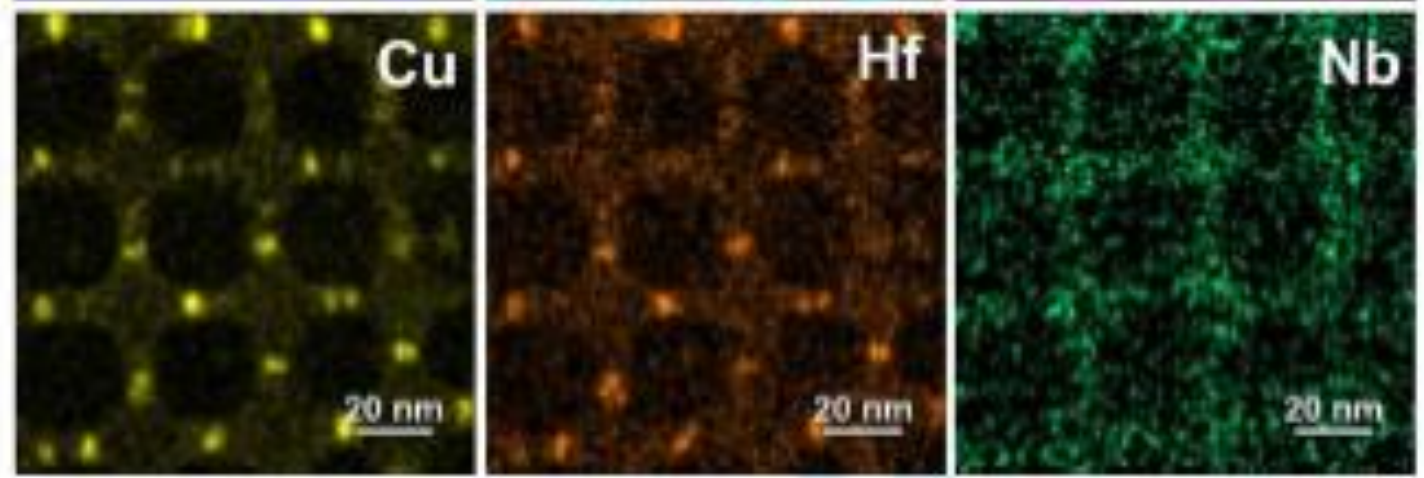

Figure 49: HAADF images along [001], and corresponding EDS maps for Sample A with Titanium (Fan et al., 2016b) 


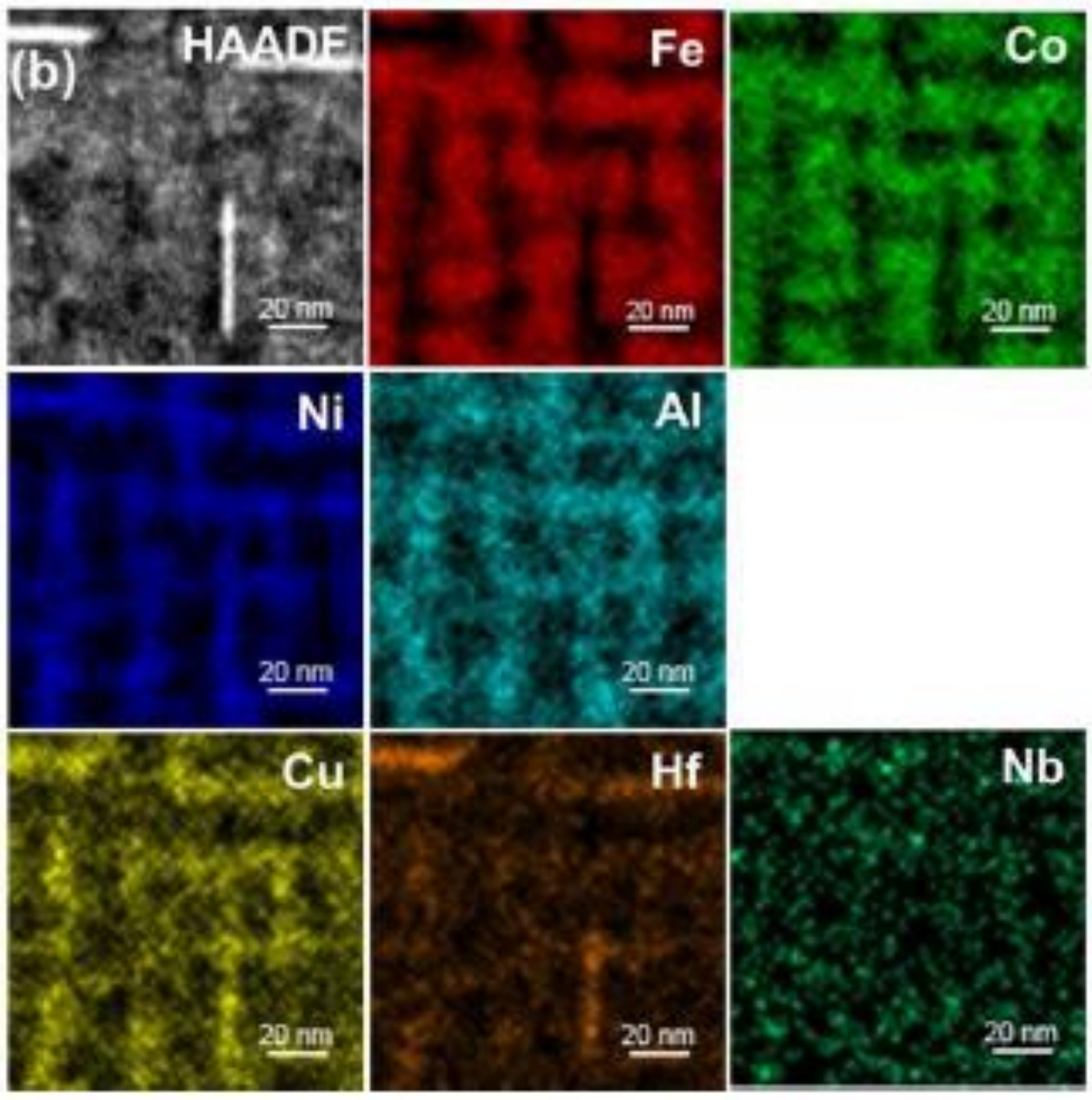

Figure 50: HAADF images along [001], and corresponding EDS maps for Sample A without Titanium (Fan et al., 2016b)

Figure 50 shows a high resolution HAADF image of Sample A with titanium. Here, the focus was around the $\alpha_{1}$ phase. EDS scans around the $\alpha_{1}$ phase points towards inhomogeneous distribution of $\mathrm{Ni}$ in $\alpha_{2}$ phase and reveals coexistence of $\mathrm{Ni}$ and $\mathrm{Cu}$ loops. This work was further expanded to characterize, $\mathrm{Cu}>\mathrm{Ni}$ and $\mathrm{Ni}>\mathrm{Cu}$ rich interrelated our hypothesis, that is interrelationship 
between $\mathrm{Ni}-\mathrm{Cu}$ loops, $\mathrm{Cu}-\mathrm{Ni}$-rich bridges and $\mathrm{Ti}$ content. The authors presented a 3D model and a corresponding 2D transverse view of $\mathrm{Cu}$-Ni-rich bridge formation process. Readers are requested to follow our published work for more information on this topic (Fan et al., 2016a, 2016b, 2016c).

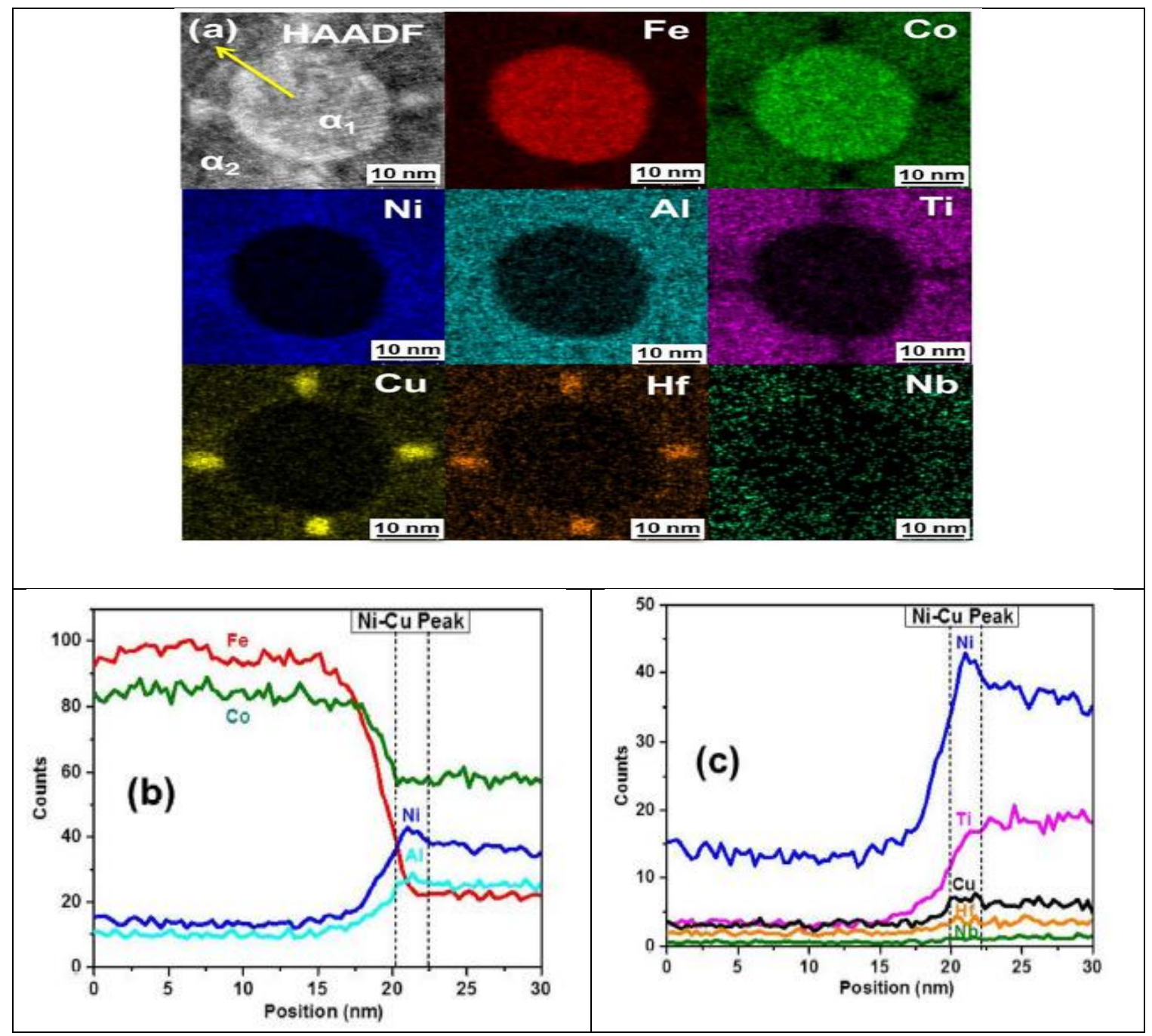

Figure 51.: High-resolution HAADF image and EDS scan for sample A with Titanium (a) HAADF image, (b) EDS scan from $\alpha_{1}$ to $\alpha_{2}$ phase, (c) EDS scan at higher resolution. (Fan et al., 2016b) 


\section{CHAPTER 8 CONCLUSIONS}

One of the main purposes of a computational materials scientist is to motivate an experimentalist to incorporate modifications in the standard alloy development protocol for improved results.

In this work, we were able to efficiently utilize limited information from the literature to develop and demonstrate a novel approach to design-optimization of high temperature, high-intensity permanent magnetic alloys. Here, we used a set of computational tools based on several concepts of artificial intelligence to develop meta-models to address composition-property relationship in multicomponent AINiCo alloys. Most of the software used in this work was developed by members of our research group.

All of the content reported here has been presented at several international conferences and has been received well by the research community. Additionally, all of the contents have been published in technical journals.

\subsection{Data-driven approach}

In this work, we were able to start from practically non-magnetic AlNiCo type chemical compositions and were able to develop strong magnetic alloys over cycles. Even though our first set of results were not acceptable, we were able to recover from it and were able to improve these properties by an order of magnitude by meta-modeling and multi-objective optimization.

Figures 5, 6 and 7 show the scatter plots of $(B H)_{\max }$ vs $H_{c}$ and $B_{r}$. The best 10 alloys are marked on these figure. The alloys were ranked on the basis of 
$(B H)_{\max }$ values in Figures 5, 6 and 7 and Table 2. So far, the best alloy is alloy 124 and its composition was predicted at MAIDROC laboratory. Pareto-optimized predictions (using modeFRONTIER, IOSO, and HYBRID approach) dominate the initial 80 candidate alloys as well as most of those randomly predicted by Sobol's algorithm in later stages. The present alloy development time was comparatively small when compared to conventional approaches. Such an approach will prove to be helpful for accelerated implementation of alloys. Hence, our approach was able to successfully recover from the initial flaws proving the robustness of this alloy design approach. This would have been impossible by random experimentation.

Obtained results were screened by using standard statistical tools and the whole work utilized multiple concepts of machine learning to arrive at a meaningful conclusion. The dataset is quite noisy; at the same time we are dealing with a multi-component system, hence the non-linear composition-property relation was expected. Nevertheless, we were able to determine a few correlations that can be proved from literature. For other correlations, we need to do further experiments. Screening of alloys on the basis of thermodynamic analysis from limited databases is another positive outcome of this work. Any other query from experimentalists can be addressed by modifying our algorithms.

Unique contributions from our collaborators can be listed as follows:

1. Significant improvement in properties for identical thermo-magnetic protocol as can be observed in Figure 52. Table 11 shows the composition of best alloy predicted so far, alloy\#124. 
a. $(\mathrm{BH})_{\max }$ : Observed $(\mathrm{BH})_{\max }$ is low, but within the bounds of $(\mathrm{BH})_{\max }$ observed in commercial AINiCo alloys.

b. $\mathrm{H}_{\mathrm{c}}$ : Observed $\mathrm{H}_{\mathrm{c}}$, is comparable to commercial AINiCo alloys.

c. $B_{r}$ : Observed $B_{r}$, is low and it is the reason for lower $(B H)_{\max }$. Hence, attempts are to be made in order to improve upon $\mathrm{B}_{\mathrm{r}}$ value.

Table 11: Chemical composition of the best optimized alloy and several commercial AINiCo alloys (Palasyuk et al. 2013)

\begin{tabular}{|c|c|c|c|c|c|c|c|c|c|c|}
\hline $\mathrm{Fe}$ & Co & $\mathrm{Ni}$ & $\mathrm{Al}$ & $\mathrm{Ti}$ & $\mathrm{Hf}$ & $\mathrm{Cu}$ & $\mathrm{Nb}$ & $(\mathrm{BH})_{\text {max }}$ & $\mathrm{H}_{\mathrm{c}}$ & $\mathrm{B}_{\mathrm{r}}$ \\
\hline \multicolumn{8}{|c|}{ Composition (Wt \%) } & $J m^{-3}$ & $\mathrm{Oe}$ & Tesla \\
\hline \multicolumn{11}{|c|}{ Chemical composition of alloy $\# 124$} \\
\hline 32.33 & 36.86 & 13.54 & 7.2 & 4.1 & 2.06 & 2.94 & 0.93 & 12072 & 1140 & 0.532 \\
\hline \multicolumn{11}{|c|}{ Chemical composition of the commercial alloy AINiCo 5-7 } \\
\hline 49.9 & 24.3 & 14.0 & 8.2 & 0.0 & 0.0 & 2.3 & 1.0 & & 740 & 1.35 \\
\hline \multicolumn{11}{|c|}{ Chemical composition of the commercial alloy AINiCo 8} \\
\hline 30.0 & 40.1 & 13.0 & 7.1 & 6.5 & 0.0 & 3.0 & 0.0 & & 1860 & 0.82 \\
\hline \multicolumn{11}{|c|}{ Chemical composition of the commercial alloy AINiCo 9} \\
\hline 35.5 & 35.4 & 13.1 & 7.0 & 5.0 & 0.0 & 3.2 & 0.5 & & 1500 & 1.06 \\
\hline
\end{tabular}




\section{$\mathrm{Br}$ vs. Hc (Second quadrant): 180 alloys}

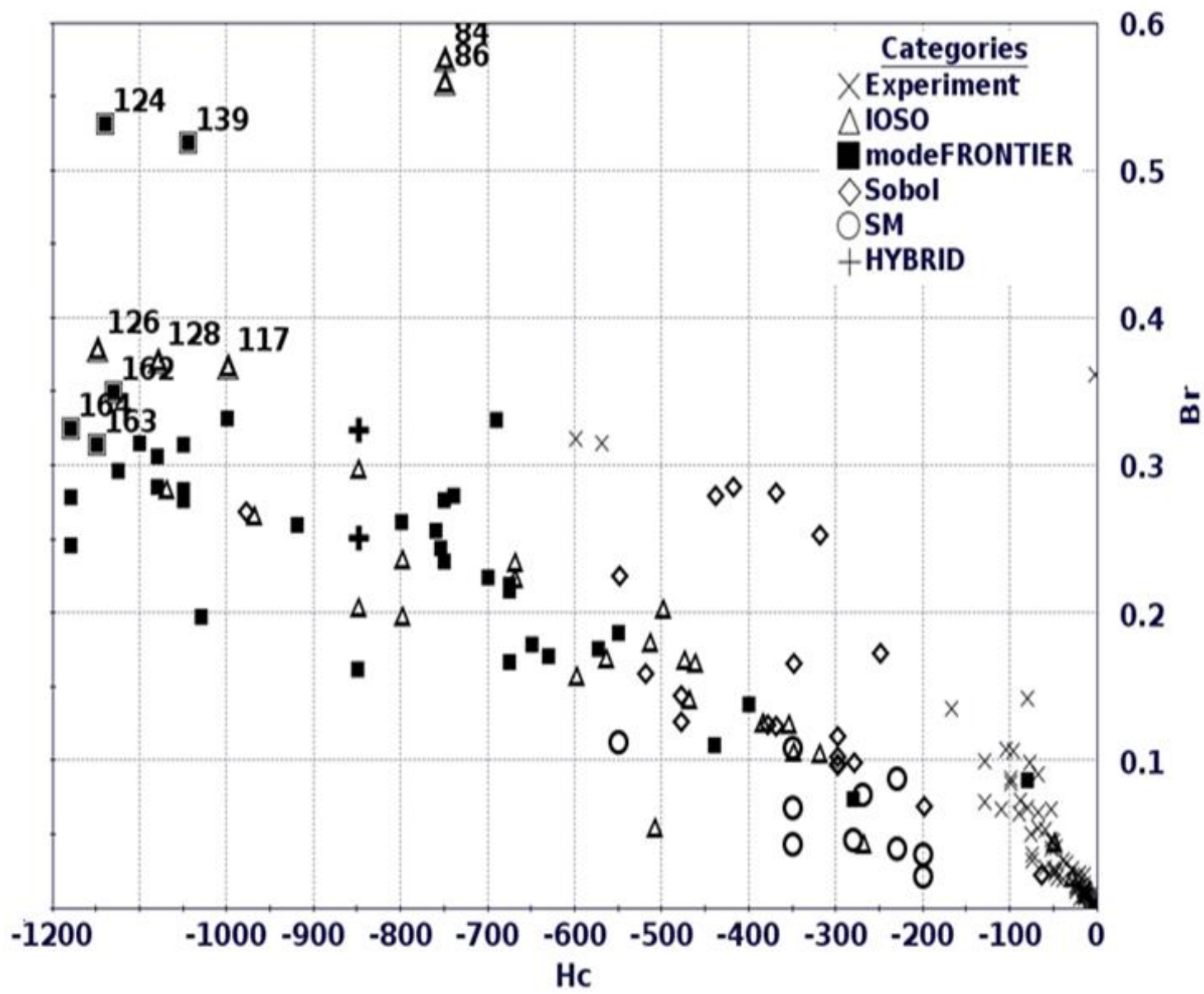

Figure 52: Scatter plot of 180 alloys on the second quadrant of $\mathrm{B}-\mathrm{H}$ curve.

2. Hf has been used for the first time in Alnico alloys. Hf is known for improving high-temperature properties. In the present case, we observed $\mathrm{Hf}$ at the grain boundaries in SEM micrographs that may enhance high temperature properties. Additionally, in HAADF images, $\mathrm{Hf}$ can be seen to be clustered on the $\mathrm{Cu}-\mathrm{Ni}$ rich bridges. At present, it is difficult to comment on the role of $\mathrm{Hf}$ on $\mathrm{Cu}-\mathrm{Hf}$ rich bridge formation, but a detailed analysis will be a novel work. 
3. Use of random number generator (Sobol's algorithm) to generate an initial set of alloys.

4. Use of multiple response surface approaches to develop meta-models, and the reason to select a particular model was well explained in this work. In this work, amongst all the algorithms available in modeFRONTIER.

a. RBF-IMQ and RBF-MQ were the best performers regarding the time taken to develop a model and accuracy of prediction.

b. Regarding the time needed to develop models, Anisotropic kriging models needed more time than RBF's while evolutionary design took the longest time. The accuracy of both these models were similar.

c. The ANN was not extensively used as the training set is too small for accurate prediction.

5. Use of several concepts of evolutionary algorithms to optimize targeted properties for deployment. In this work, amongst all the algorithms:

a. MOPSO and MOSA were the best performers regarding its ability to generate a diverse set of composition.

b. NSGA2 and its variants were used in every cycle and one of the top 10 alloys were predicted by it. It was observed that the composition of Paretooptimized predictions in later cycles were almost identical that is it differed in third place of decimal. It is very difficult for an experimentalist to manufacture such alloys.

6. Use of Adaptive Space Filler (ASF) Algorithm as DOE: ASF was used to predict compositions that acted as a starting point in all the optimization cycles in order 
extensively search the variable space for diverse composition. Sobol's algorithm was also tried, but ASF yields a more diverse set.

7. Use of MCDM, PCA and HCA as screening tools to manufacture alloys for the next cycle.

a. MCDM: modeFRONTIER was used for this work. Genetic algorithm module provided with better results in comparison to other approaches available in the toolbox

8. Use PCA and HCA to find various patterns within the dataset.

a. PCA: modeFRONTIER and IBMSPSS were both used and provides similar results. $\mathrm{R}$ or Weka can be used as an open source alternative for similar work.

b. HCA: I preferred modeFRONTIER due to better graphics in comparison to IBMSPSS. In HCA, Ward's approach yields the best results among all the algorithms available in modeFRONTIER.

9. The use of HCA to predict optimized composition of a few elements.

10. This approach can be beneficial for other systems of alloys for design and accelerated deployment. We have tested our approach on Nickel based superalloys. These approaches can be coupled together and will help in taking critical decisions needed during alloy design in terms of alloy chemistry or manufacturing protocol. Thus, such an approach will help in moving a step further, that is, towards realizing virtual material design paradigm for the design and accelerated deployment of alloys for targeted properties. 


\subsection{Experiments}

Unique contributions from our collaborators can be listed as follows:

1. Design thermo-magnetic protocol that helped to increase in $H_{c}$.

2. Study on the evolution of $\mathrm{Cu}-\mathrm{Ni}$ rich bridges in AINiCo alloys.

3. Study effect of tempering on the formation and growth of Cu-Ni rich bridges.

\subsection{Future works}

Due to funding and time constraints, we were not able to address a few issues that we think is important for this problem. Future work will be focussed on:

1. Improvement of response surface accuracy.

2. Introduce aleatory and epistemic uncertainty in response surface predictions.

3. Make attempts to address uncertainty propagation.

4. Use K-optimality criterion in order to optimize more than three objectives at a time.

5. Evaluate the scope of rare-earth additions. We have started work in this regard and used an ab-initio based database, Materials Project (Materials Project, 2014) to perform some calculations. Table 1 shows the result of these calculations where we basically highlighted the magnetic moment of various structures. Here we can observe that there exist unstable structures with superior magnetic moment as compared to the stable structures for Cerium addition. Thus, from these results we can think of Cerium additions to improving the magnetic properties. This will be economical as Cerium is a non-critical REE. The major challenge is to stabilize the structures with superior magnetic 
moment. Hence, we need to design a thermomagnetic protocol in order to stabilize the unstable structures.

Table 12: Evaluating the scope of REE (Cerium) addition

\begin{tabular}{|l|l|l|l|}
\hline Phase & Magnetic moment $(\mu \beta)$ & Magnetic ordering & Decomposes to \\
\hline $\mathrm{Ce}_{2} \mathrm{C}_{17}$ & 46.262 & Unknown & $\mathrm{CeC}{ }_{2}+\mathrm{Co}$ \\
\hline $\mathrm{CeC}{ }_{3}$ & 6.023 & Unknown & $\mathrm{CeC}{ }_{2}+\mathrm{Co}$ \\
\hline $\mathrm{CeC}{ }_{5}$ & 5.749 & Unknown & $\mathrm{CeC}_{2}+\mathrm{Co}$ \\
\hline $\mathrm{CeC}_{2}$ & 0.00 & Unknown & Stable \\
\hline $\mathrm{Ce}_{2} \mathrm{Fe}_{17}$ & 37.075 & Unknown & $\mathrm{CeFe} 2+\mathrm{Fe}$ \\
\hline $\mathrm{CeFe}_{5}$ & 9.864 & Unknown & $\mathrm{CeFe} 2+\mathrm{Fe}$ \\
\hline $\mathrm{CeFe}_{2}$ & 5.015 & Unknown & Stable \\
\hline
\end{tabular}

6. Work on multi-scale modelling for development of magnets. Finally, our purpose is to combine all the above so as to find correlations between various scales of modelling, minimize propagation of uncertainty between the scales, so as to improve meta-model prediction for accelerated deployment of these alloys. 


\section{REFERENCES}

Bale, C., Chartrand, P., Degterov, S., Eriksson, G., Hack, K., Mahfoud, R. B., Melançon, J., Pelton, A., and Petersen, S. (2002). Factsage thermochemical software and databases. Calphad, 26(2), pp. 189-228.

Basheer, I.A. and Hajmeer, M. (2000). Artificial neural networks: fundamentals, computing, design and application, Journal of Microbiological Methods, 43, pp. 3-31

Box, G. E. P., and Draper, N. R. (1987) Empirical Model-Building and Response Surfaces, . Wiley, New York.

\section{Earths.html}

Cen-ACS (2016), http://cen.acs.org/articles/93/i30/Struggle-Mine-Rare-

Cullity, B. and Graham, C. (2009). Chapter 14. Hard magnetic materials. In Introduction to Magnetic Materials, Second Edition, pp. 477-504. Wiley-IEEE Press, New York.

Deb, K. (2001). Multi-objective Optimization Using Evolutionary Algorithms. John Wiley and Sons, Chichester, UK.

Dilon, H. (2014). Effects of heat treatment and processing modifications on microstructure in AINiCo-8h permanent magnetic alloys for high temperature applications. Graduate thesis and dissertations, lowa State University, http:/lib.dr.iastate.edu/etd/13867. Paper 13867.

Dulikravich, G. S. and Colaço, M. J. (2015). Hybrid Optimization Algorithms and Hybrid Response Surfaces, Chapter 2 in Advances in Evolutionary and Deterministic Methods for Design, Optimization and Control in Engineering and Sciences (eds.: D. Greiner, B. Galván, J. Periaux, N. Gauger, K. Giannakoglou, G. Winter), Computational Methods in Applied Sciences Series, Springer Verlag, 2015, pp. 19-47. ISBN: 978-3-319-11541-2 DOI: 10.1007/9783-319-11541-2_2

Egorov-Yegorov, I. N. and Dulikravich, G. S. (2005). Chemical composition design of superalloys for maximum stress, temperature, and time-torupture using self-adapting response surface optimization. Materials and Manufacturing Processes, pp. 569-590.

EPA (2012), The White House, Office of the Press Secretary,

https://www.whitehouse.gov/the-press-office/2012/08/28/obamaadministration-finalizes-historic-545-mpg-fuel-efficiency-standard 
FACTSAGE, (2015), http://www.crct.polymtl.ca/factsage/fs equilib.php,

Fan, M., Liu, Y., Jha, R., Dulikravich, G. S., Schwartz, J., and Koch, C. C. (2016a). Effect of Cu-Ni-rich Bridges on the Microstructure and Magnetic Properties of Alnico Alloys, IEEE Transactions on Magnetics, pp. (99):1-1, DOI: 10.1109/TMAG.2016.2555956.

Fan, M., Liu, Y., Jha, R., Dulikravich, G., Shwartz, J., and Koch, C. C. (2016b). Microscopic characterization of cu-ni-rich bridges in AINiCo alloys. In 13th Joint MMM-Intermag Conference, San Diego, CA., U.S.A.

Fan, M., Liu, Y., Jha, R., Dulikravich, G. S., Schwartz, J., and Koch, C. C. (2016c). On the Evolution of Cu-Ni-rich Bridges of Alnico Alloys with Tempering, Journal of Magnetism and Magnetic Materials, July 2016, DOI: 10.1016/j.jmmm.2016.07.040.

Giri, B. K., Hakanen, J., Miettinen, K., Chakraborti, N., (2013). Genetic programming through bi-objective genetic algorithms with a study of a simulated moving bed process involving multiple objectives, Applied Soft Computing, 13: 5, pp. 2613-2623.

Horstemeyer, M. F. (2012). Integrated Computational Materials Engineering (ICME) for Metals: Using Multiscale Modeling to Invigorate Engineering Design with Science. TMS - The Minerals, Metals and Materials Society, John Willey and Sons, Inc.), Hoboken, New Jersey. 2015

IBM SPSS,(2015a), https://en.wikipedia.org/wiki/SPSS, accessed on 4-21-

IBMSPSS (2015): IBM corp. released 2013. IBM spss statistics for windows, version22.0.armonk,ny:lbmcorp.,

http://www-

01.ibm.com/software/analytics/spss/, accessed on 3/1/2015.

Jha, R., Dulikravich, G. S., Colaço, M. J., Egorov, I. N., Poloni, C., Chakraborti, N., Fan, M., Shwartz, J., and Koch, C. C. (2015a). Magnetic alloys design using multi-objective optimization. MS\&T15-Materials Science and Technology 2015 Conference, Columbus, Ohio, October 4-8, $2015 .$.

Jha, R., Dulikravich, G. S., Colaço, M. J., Fan, M., Shwartz, J., and Koch, C. C., (2016). Magnetic Alloys Design Using Multi-Objective Optimization, to appear in Advanced Structured Materials series, Vol. 33, (eds.: Oechsner, A., da Silva, L.M., Altenbach, H.), Properties and Characterization of Modern Materials, Springer, Germany, 978-981-10-1601-1, 421721_1_En, (22).

Jha, R., Dulikravich, G. S., Chakraborti , N., Fan, M., Shwartz, J., and Koch, 
C. C., Colaço, M. J., Poloni, C., Egorov, I. N., (2016). Algorithms for design optimization of chemistry of hard magnetic alloys using experimental data, Journal of Alloys and Compounds, Vol. 682, 2016, pp. 454-467, DOI: 10.1016/j.jallcom.2016.04.218.

Jha, R., Pettersson, F., Dulikravich, G. S., Saxen, H., and Chakraborti, N. (2015b). Evolutionary design of nickel-based superalloys using data-driven genetic algorithms and related strategies. Materials and Manufacturing Processes, 30: pp. 488-510.

Jha, R., Pettersson, F., Dulikravich, G. S., Saxen, H., and Chakraborti, N. (2014a), A combined experimental-computational approach to design optimization of high temperature alloys. In: ASME Symposium on Elevated Temperature Application of Materials for Fossil, Nuclear, and Petrochemical Industries, ASME, Seattle, WA, March 25-27, 2014.

Jha, R., Sen, P. K., and Chakraborti, N. (2014). Multi-objective genetic algorithms and genetic programming models for minimizing input carbon rates in a blast furnace compared with a conventional analytic approach. Steel Research International, 85: pp. 219-232.

Jha, R., Dulikravich, G. S., Colaço, M. J., Design and optimization of magnetic alloys and nickel-based superalloys for high temperatures applications, COBEM-2015, paper 1284, Rio de Janeiro, Brazil, 6-11 December, 2015.

Jha, R., Dulikravich, G. S., Colaço, M. J., Fan, M., Shwartz, J., and Koch, C. C., Magnetic alloys design using multi-objective optimization, ACEX2015-9th International Conference on Advanced Computational Engineering and Experimenting, Munich, Germany, June 29 - July 22015.

Jha, R., Dulikravich, G. S., Chakraborti , N., Fan, M., Shwartz, J., and Koch, C. C., Colaço, M. J., Poloni, C., Egorov, I. N. Algorithms for multi-objective design optimization of hard magnetic alloys using experimental data, ICMM4-International Conference on Material Modeling, Berkeley, CA, May 27-29, 2015.

Jha, R., Dulikravich, G. S., Fan, M., Shwartz, J., Koch, C. C., Egorov, I. N., Poloni, C., A combined computational-experimental approach to design of highintensity permanent magnetic alloys, CONEM2014- National Congress of Mechanical Engineering (eds: Steffen, V., Bandarra, E., Rade, D. A.), Uberlandia, Brazil, August 10-15, 2014.

Kennedy, J. and Eberhart, R. (1995), Particle Swarm Optimization. Proceedings of IEEE International Conference on Neural Networks. pp. 19421948. doi:10.1109/ICNN.1995.488968. 
Kirkpatrick, S., Gelatt Jr, C. D., Vecchi, M. P. (1983). Optimization by simulated annealing. Science 220 (4598), pp. 671-680

M\&M, 2016, http://www.marketsandmarkets.com/Market-Reports/magneticmaterials-397.html

Kramer, M. J., McCallum, R. W., Anderson, I. A., and Constantinides, S. (2012). Prospects for non-rare earth permanent magnets for traction motors and generators. JOM, 64, pp. 752-763.

Materials Project (2014) https://www.materialsproject.org/

Mcguiness, P., Akdogan, O., Asali, A., Bance, S., Bittner, F., Coey, J. M. D., Dempsey, N. M., Fidler, J., Givord, D., Gutfleisch, O., Katter, M., Le Roy, D., Sanvito, S., Schrefl, T., Schultz, L., Schwöbl, C., Soderžnik, M., Šturm, S., Tozman, P., Üstüner, K., Venkatesan, M., Woodcock, T. G., Žagar, K., and Kobe, S. (2015). Replacement and Original Magnet Engineering Options (ROMEOs): A european seventh framework project to develop advanced permanent magnets without, or with reduced use of, critical raw materials, JOM, 67, pp. 1306-1317.

Metropolis, N., Rosenbluth, N., Rosenbluth, M., Teller, A., and Teller, E. (1953). Equation of state calculations by fast computing machines. Journal of Chemical Physics, 21, pp.1087-1092.

Mueller, T., Kusne, A., and Ramprasad, R. (2015). Machine learning in materials science: Recent progress and emerging applications. Rev. Comput. Chem.(Accepted for publication).

Palasyuk, A., Blomberg, E., Prozorov, R., Yue, L., Kramer, M. J., McCallum, R. W., Anderson, I. E., and Constantinides, S. (2013), Advances in characterization of non-rare-earth permanent magnets: exploring commercial alnico grades 5-7 and 9, JOM, Volume 65, Issue 7, pp. 862-869.

Panchal, J. H., Kalidindi, S. R., and McDowell, D. L. (2013). Key computational modeling issues in integrated computational materials engineering. Computer-Aided Design, 45(1) pp. 4-25.

Pettersson, F., Chakraborti, N., and Saxén, H. (2007). A genetic algorithms based multi-objective neural net applied to noisy blast furnace data. Applied Soft Computing Journal, 7(1), pp. 387-397.

Poli, R., Langdon, W. B., and McPhee, N. F. (2008), A Field Guide to Genetic Programming, ISBN 9781409200734

R (2016), https://en.wikipedia.org/wiki/R (programming language) 
Rajan, K. (2013). Materials informatics: An introduction. Informatics for materials science and engineering: data-driven discovery for accelerated experimentation and application, pp. 1-16.

Rettig, R., Ritter, N. C., Helmer, H. E., Neumeier, S., and Singer, R. F. (2015). Single-crystal nickel-based superalloys developed by numerical multicriteria optimization techniques: design based on thermodynamic calculations and experimental validation. Modelling and Simulation in Materials Science and Engineering, 23(3), pp. 035004.

Ronning, F. and Bader, S. (2014). Rare earth replacement magnets. Journal of Physics: Condensed Matter, 26, pp.1-3.

Rechenberg, I. (1971): Evolutionsstrategie - Optimierung technischer Systeme nach Prinzipien der biologischen Evolution ( $\mathrm{PhD}$ thesis). Reprinted by Fromman-Holzboog (1973).

Schwefel, H.-P. (1974): Numerische Optimierung von Computer-Modellen (PhD thesis). Reprinted by Birkhäuser (1977).

Sellmyer, D. J., Balamurugan, B., Zhang, W. Y., Das, B., Skomski, R., Kharel, P., and Liu, Y. (2013). Advances in rare-earth-free permanent magnets. In the 8th Pacific Rim International Congress on Advanced Materials and Processing.

Settouti, N. and Aourag, H. (2015). A study of the physical and mechanical properties of lutetium compared with those of transition metals: A data mining approach. JOM(The Journal of the Minerals, Metals and Materials society(TMS)), 67, pp. 87-93.

Sobol, I. (1967). On the distribution of points in a cube and the approximate evaluation of integrals. USSR Computational Mathematics and Mathematical Physics, 7(4), pp. 86-112.

Thermocalc (2015): http://www.thermocalc.com/solutions/byapplication/alloy-development/, accessed on 3/1/2015.

Toda-Caraballo, I. and Rivera-Diaz-Del-Castillo, P. (2015). Modelling and design of magnesium and high entropy alloys through combining statistical and physical models. JOM (The Journal of the Minerals, Metals and Materials society(TMS)), 67, pp. 108-117.

Ward, J. H., Jr. (1963), "Hierarchical Grouping to Optimize an Objective Function", Journal of the American Statistical Association, 58, 236-244. 
WEKA (2016), https://en.wikipedia.org/wiki/Weka_(machine_learning)

Xing, Q., Miller, M. K., Zhou, L., Dillon, H. M., McCallum, R. W., and Anderson, I. E. (2013). Phase and elemental distributions in AlNiCo magnetic materials. IEEE Transactions on Magnetics, 49, pp. 3314-3317.

Zhou, L., Miller, M., Lu, P., Ke, L., Skomski, R., Dillon, H., Xing, Q., Palasyuk, A., McCartney, M., Smith, D., Constantinides, S., McCallum, R., Anderson, I., Antropov, V., and Kramer, M. (2014). Architecture and magnetism of AlNiCo. Acta Materialia, 74, pp. 224-233. 
VITA

\section{RAJESH JHA}

Born in Bihar, India

2005- B.Sc. (Metallurgical Engineering),

2009 Bihar Institute of Technology, Sindri, Dhanbad, India

2009- Project Assistant (Applied Chemistry and Corrossion)

2010 National Metallurgical Laboratory, Jamshedpur, Jharkhand, India

2010- M. Tech. (Metallurgical and Materials Engineering),

2012 Indian Institute of Technology, Kharagpur, India

2012- Assistant Professor (Metallurgy), OP Jindal University, Raigarh, CG, India 2012

AFOSR Research Fellowship

Ph.D. Candidate, Materials Science and Engineering, FIU

2013- Visiting Researcher, Abo Akademi University, Abo, Finland

2016 AIST Steel to Students Award (7 Technical Training Conferences)

FIU-GPSC Travel Allowance to present paper at MS\&T 2015

FIU-UGS Degree Year Fellowship for Spring and Summer 2016

\section{PUBLICATIONS}

1. R. Jha, G. S. Dulikravich, M. J. Colaco, M. Fan, J. Schwartz, C. C. Koch; Magnetic Alloys Design Using Multi-Objective Optimization, Advanced Structured Materials series, Vol. 33, (eds.: A. Oechsner, L.M. da Silva, H. Altenbach), Properties and Characterization of Modern Materials, 978-98110-1601-1, 421721_1_En, (22), Springer, Germany.

2. R. Jha, G. S. Dulikravich, N. Chakraborti, M. Fan, J. Schwartz, C. C. Koch, M. J. Colaco, C. Poloni, I. N. Egorov, " Algorithms for Design Optimization of Chemistry of Hard Magnetic Alloys Using Experimental Data " Journal of Alloys and Compounds, Vol. 682, pp. 454-467, 2016, DOI: 10.1016/j.jallcom.2016.04.218. 
3. M. Fan, Y. Liu, R. Jha, G. S. Dulikravich, J. Schwartz and C. C. Koch, "Effect of Cu-Ni-rich Bridges on the Microstructure and Magnetic Properties of Alnico Alloys", IEEE Transactions on Magnetics, Vol 52, No, 8, pp. 1-10, August 2016, DOI: 10.1109/TMAG.2016.2555956.

4. M. Fan, Y. Liu, R. Jha, G. S. Dulikravich, J. Schwartz and C. C. Koch, "On the Evolution of Cu-Ni-rich Bridges of Alnico Alloys with Tempering", Journal of Magnetism and Magnetic Materials, July 2016, DOI: 10.1016/j.jmmm.2016.07.040.

5. C.C. Pacheco, M. Vesenjak, M. Borovinsek, R. Jha, S.R. Reddy, G.S. Dulikravich, H.R.B. Orlande and M.J. Colaco; "Inverse Parameter Identification Using Bayesian Statistics and Response Surfaces", Technische Mechanik, 36, 1-2, pp. 120 - 131, January 2016.

6. P. S. Babu, R. Jha, M. Guzman, G. Sundararajan and A. Agarwal, "Indentation Creep Behavior of Cold Sprayed Aluminum Amorphous/Nanocrystalline Coatings", Material science and Engineering A, Vol. 658, pp. 415-421, March 2016, DOI: 10.1016/j.msea.2016.02.030.

7. R. Jha, F. Pettersson, G. S. Dulikravich, H. Saxen, and N. Chakraborti, "Evolutionary Design of Nickel-Based Superalloys Using Data-Driven Genetic Algorithms and Related Strategies", Materials and Manufacturing Processes, Vol:30, Issue:4, pp. 488-510, April 2015.

8. R. Jha, P. Sen, N. Chakraborti, "Multi-Objective Genetic Algorithms and Genetic Programming Models for Minimizing Input Carbon Rates in a Blast Furnace Compared with a Conventional Analytic Approach", Steel Research International Vol:85, Issue: 2, pp. 219-232 January 2014

9. K. Nayak and R. Jha, "Optimizing carbon rate and hot metal temperature of an operating blast furnace by means of data driven models", Jindal Tech, September 2013.

10. (Acknowledged) R. Singh et al., "Erosion and Corrosion Behavior of Laser Cladded Stainless Steels with Tungsten Carbide" Journal of Materials Engineering and Performance, October 2012.

11.S. R. Reddy, A. Abdoli, G.S. Dulikravich, C. C. Pacheco, G. Vasquez, R. Jha, M. J. Colaco and H. R. B. Orlande, "Multi-Objective Optimization of Micro Pin-Fin Arrays for Cooling of High Heat Flux Electronics with a Hot spot", accepted for Heat Transfer Engineering.

12. S.R. Reddy, A. Abdoli, G.S. Dulikravich, R. Jha, "Conjugate Analysis of Thin Film Heat Spreaders to Reduce Temperature at Hot Spots", ASME Paper No. HTE-10015. (under review). 